\title{
ON THE SECOND RATIONAL $K$-GROUP OF AN ELLIPTIC CURVE OVER GLOBAL FIELDS OF POSITIVE CHARACTERISTIC
}

\author{
SATOSHI KONDO AND SEIDAI YASUDA
}

\begin{abstract}
Let $E$ be an elliptic curve over a global field of positive characteristic. Let $r$ be the order of zero at $s=0$ of the Hasse-Weil $L$-function with bad factors removed. The Parshin conjecture on the vanishing of higher rational $K$ theory of projective smooth schemes over finite fields implies $\operatorname{dim}_{\mathbb{Q}} K_{2}(E) \otimes_{\mathbb{Z}}$ $\mathbb{Q}=r$. It is shown that $\operatorname{dim}_{\mathbb{Q}} K_{2}(E) \otimes_{\mathbb{Z}} \mathbb{Q} \geq r$.
\end{abstract}

(This preprint is a revised version of the first half of our preprint RIMS-1564, RIMS, Kyoto University, August 2006.)

\section{INTRODUCTION}

Let $C$ be a connected projective smooth curve over a finite field. Let $\mathcal{E} \rightarrow C$ be an elliptic surface, that is, a proper, flat, generically smooth morphism from a regular scheme $\mathcal{E}$ to $C$ such that for almost all closed points $\wp$ of $C$, the fiber $\mathcal{E} \times{ }_{C} \wp$ is a genus 1 curve. Let $E$ denote the generic fiber of $\mathcal{E}$.

For a closed point $\wp$ of $C$, let $\kappa(\wp)$ be the residue field at $\wp$ and $\mathcal{E}_{\wp}=\mathcal{E} \times_{C}$ $\operatorname{Spec} \kappa(\wp)$ be the fiber at $\wp$. Let us identify the $K$-theory and the $G$-theory of regular noetherian schemes. There is a localization sequence of $G$-theory:

$$
K_{2}(\mathcal{E}) \rightarrow K_{2}(E) \stackrel{\oplus \partial_{\wp}}{\longrightarrow} \bigoplus_{\wp} G_{1}\left(\mathcal{E}_{\wp}\right) \rightarrow K_{1}(\mathcal{E})
$$

where $\wp$ runs over all closed points of $C$.

We use the subscript $-\mathbb{Q}$ to mean $-\otimes_{\mathbb{Z}} \mathbb{Q}$.

Theorem 1.1. Let the notations be as above. Then the homomorphism

$$
K_{2}(E)_{\mathbb{Q}} \stackrel{\oplus \partial_{\wp \mathbb{Q}}}{\longrightarrow} \bigoplus_{\wp} G_{1}\left(\mathcal{E}_{\wp}\right)_{\mathbb{Q}}
$$

is surjective.

Let $n$ be a positive integer. The Parshin conjecture says that $K_{n}(X)_{\mathbb{Q}}=0$ for any smooth projective variety $X$ over a finite field. The validity of the conjecture in particular implies that $K_{1}(\mathcal{E})_{\mathbb{Q}}=K_{2}(\mathcal{E})_{\mathbb{Q}}=0$, hence that the homomorphism in Theorem 1.1 is an isomorphism. One can compute exactly the target space when $E$ is an elliptic curve (see Lemma 2.2).

During this research, the first author was supported as a Twenty-First Century COE Kyoto Mathematics Fellow, by World Premier International Research Center Initiative (WPI Initiative), MEXT, Japan, and was partially supported by JSPS Grant-in-Aid for Scientific Research 17740016. The second author was partially supported by JSPS Grant-in-Aid for Scientific Research 16244120 . 
Let $E$ be an elliptic curve over a global field $k$ of positive characteristic. The first author's principal motivation was to prove the following corollary, which we will deduce from Theorem 1.1 in Section 11 by taking $C$ to be the projective smooth curve over a finite field whose function field is $k$ and $\mathcal{E}$ to be the flat proper regular minimal model of $E$ over $C$. For a finite set $M$, we let $|M|$ denote the cardinality of $M$.

Corollary 1.2. Let $S$ be the set of primes of $k$ at which $E$ has split multiplicative reduction. Then $\operatorname{dim}_{\mathbb{Q}} K_{2}(E)_{\mathbb{Q}} \geq|S|$.

Let $L^{S}(E, s)$ denote the Hasse-Weil $L$-function of $E$ with the local factors at the primes in $S$ removed. Then Corollary 1.2 gives a relation between the rank of $K_{2}(E)$ and the $L$-function $L^{S}(E, s)$, since the order of zero at $s=0$ of $L^{S}(E, s)$ is equal to $|S|$.

The analogue of Corollary 1.2 over number fields is that the rational rank of the $K$-group is greater than or equal to $|S|+[k: \mathbb{Q}]$ (see [Ro-Sc, Section 1.2, p. 62]). It is a consequence of (a strong form of) Beilinson's conjectures, and is not yet proved. Bloch and Grayson give a method ([Bl-Gr, p. 81-83]) for constructing elements in $K_{2}$ such that the image under the boundary map is not trivial, but one requires the condition that the image of $\operatorname{Gal}(\bar{k} / k) \rightarrow \prod_{l} \operatorname{End}_{\mathbb{Z}_{\ell}}\left(T_{\ell}(E(\bar{k}))\right.$ ) (where $\bar{k}$ is the separable closure of $k$ and $\ell$ runs over all primes) is small. Hence their method is not applicable in general.

As an application, we see that there exists an elliptic curve over a global field of positive characteristic with arbitrarily large rational $K_{2}$.

Corollary 1.3. Let $k$ be a global field of positive characteristic. Then for any integer $n \geq 0$, there exists an elliptic curve $E$ over $k$ such that $\operatorname{dim}_{\mathbb{Q}} K_{2}(E)_{\mathbb{Q}} \geq n$.

We have another application of Theorem 1.1. From the theorem, we infer that the cokernel of $\oplus \partial_{\wp}$ is torsion. We show in our forthcoming paper that the torsion group is a finite group, and that the order is explicitly determined when $\mathcal{E} \rightarrow C$ is non-isotrivial and has a section.

To prove Theorem 1.1 we use modular parameterizations of $E$ by Drinfeld modular curves and the analogue of Beilinson elements, constructed in [Ko-Ya], for Drinfeld modular curves. The key idea is that, for every place $\wp$ at which the elliptic curve has split multiplicative reduction, one has a modular parametrization of $E$ by a Drinfeld modular curve where $\wp$ plays the role of the archimedean place of $\mathbb{Q}$, and hence one has an analogue of Beilinson elements for every such $\wp$. The linear independence of the elements amounts to the integrality of the elements.

The sections are organized as follows. Sections 2 and 3 do not depend on other sections. The output are Proposition 2.1, Lemma 2.2 and Proposition 3.1. The theme of these two sections is to compare the G-group of the special fiber (resp. the boundary map from the generic fiber) and the étale cohomology of the generic fiber (resp. the Chern class map). Sections 4, 5, and 6 compute the moduli of Drinfeld modular curves. Recall that there is an analogy between the moduli of elliptic curves and the Drinfeld modular curves. The analogous statements given in these three sections (see the next paragraphs for the contents) are well known for the case of elliptic modular curves. We supply the proofs for our Drinfeld modular case since we were not able to find a good reference. The results of these three sections will be used in Section 7. Sections 7, 8, 9, and 10 contain the Drinfeld modular analogue of the work of Beilinson ([Be], see also the book $[\mathrm{RSS}])$. We refer 
to the introduction in Section 7 for a very short paragraph on Beilinson's result. Section 11 contains the idea which does not have an analogue in the number field case, namely to vary the modular parametrizations to construct (many) elements in the rational $K_{2}$ of the elliptic curve.

We give the contents of each Section below. The reader is referred to the beginning of each Section for more technical details.

In Section 2, we compute the target group of the homomorphism $\oplus_{\wp} \partial_{\wp} \mathbb{Q}$ of Theorem 1.1. If one is interested only in the minimal model, the proof is easy (we refer to [Bl-Gr, p.82-83]). The reader may read only the statements of Proposition 2.1 and Lemma 2.2 and skip the proof.

Section 3 contains Proposition 3.1. This is a rather technical statement, and the proof is again a lot of diagram chasing in (co)homology theories. We explain how Proposition 3.1 will be used. The main ingredient for the proof of Theorem 1.1 is Lemma 8.1, which is stated in terms of the regulator (which is close to the boundary map to the special fiber). We use Proposition 3.1 to convert it to the statement solely in terms of generic fiber, that is, in a "model free" manner. The reader may skip the technical details of this section.

In Section 4, we construct what is called the determinant morphism. This is a morphism from the moduli of Drinfeld modules of rank $d$ to that of rank 1 . The only case of our interest is the case $d=2$; this may be regarded as the analogue of the morphism obtained from the Weil pairing in the case of ellitpic curves. Although we do not construct the actual pairing, we mention that the construction of the analogue of the Weil pairing for Drinfeld modules have been done by Hamahata [Ha] for the rank two Drinfeld modules over the base ring $A=\mathbb{F}_{q}[t]$ using the theory of $t$-motives, and by van den Heiden [vdHe] for general rank over the general base $A$ using the theory of $A$-motives. We also give the details on the uniformization of the moduli space using elliptic sheaves. See the beginning of the section for more information.

In Section 5, we give the details on an analogue of Deuring's theory on supersingular elliptic curves for Drinfeld modules of rank two. The essential part of the results is due to Drinfeld; there are also papers [Gek4], [Gek5], [Gek3] by Gekeler. The output of this section are the isomorphism (5.4) and Proposition 5.4. We describe the set of supersingular points as a double coset space by introducing a quaternion algebra (obtained from the endomorphism ring of a supersingular Drinfeld module). The statements are standard in the case of moduli of elliptic curves; the reader who is familiar with them may skip the details of this section.

In Section 6, we describe the reduction modulo a prime of Drinfeld modular curve. Analogous statements for elliptic modular curves are found in the book [Ka-Ma]. We give the details for we were not able to find them in the literature.

In Section 7, we prove the integrality of certain elements in the $K_{2}$ of Drinfeld modular curves. This section is the function field analogue of [Sc-Sc, Section 7, p. 298-302].

The aim of Section 8 is to quote a theorem in our other paper [Ko-Ya]. In the course, we describe the Bruhat-Tits building (which is closely related to the Drinfeld symmetric space) and a "regulator" map (which is a map from the Kgroup of the moduli space to the space of automorphic forms). Lemma 8.1 roughly says that there exists a subspace in the rational $K_{2}$ of the Drinfeld modular curve which maps surjectively to the space of harmonic cochains. 
In Section 9, we apply Lemma 8.1 and prove Proposition 9.1. This roughly says that there exists a certain subspace in the rational $K_{2}$ of the compactified Drinfeld modular curve.

In Section 10, we recall the modular parametrization of an elliptic curve over a function field by a (compactified) Drinfeld modular curve. We apply this (Section 11.1) to construct certain elements in the rational $K_{2}$ of the elliptic curve from the elements in the rational $K_{2}$ of the compactified Drinfeld modular curve of Section 9 .

In the final Section 11, we give proofs of Theorem 1.1 and Corollaries 1.2 and 1.3 .

Acknowledgment The first author is grateful to Professor Bloch for numerous discussions and to the University of Chicago for hospitality. He also thanks Masanori Asakura for his interest, Fumiharu Kato for comments on rigid analysis, and Takeshi Abe for discussions.

The second author would like to thank Teruyoshi Yoshida for discussions about bad reductions of modular varieties.

The authors would like to thank Takuya Yamauchi for his suggestion of the statement of Corollary 1.3, and the referee for his/her comments which improved our article.

\section{The rational G-Group of the REDUCtion}

The aim of this section is to compute the target space of the homomorphism $\oplus_{\wp} \partial_{\wp_{Q}}$ in Theorem 1.1. When $E$ is an elliptic curve, i.e., when the canonical morphism $E \rightarrow$ Spec $k$ has a section, the result is Lemma 2.2. This will only be used in Section 11.1. For the proof of Lemma 2.2, we use Proposition 2.1. This will turn out to be useful in the proof of Lemma 11.2.

The result (Lemma 2.2) is very simple but the proof is long. If one is to assume that the model $\mathcal{E}$ is a minimal model, then the computation is easy, since the rank of the rational $G_{1}$ is then equal to the rank of the first homology of the dual graph (see [Bl-Gr, p.82-83]). The reader interested only in the minimal model case may skip the entire section.

2.1. We give a proof of the following proposition in Sections 2.1.1-2.1.5.

Let $S$ be the spectrum of a henselian discrete valuation ring whose residue field is a finite field of characteristic $p$. We denote by $s$ (resp. $\eta$ ) the closed (resp. generic) point in $S$. Let $X \rightarrow S$ be a proper flat morphism from a regular scheme $X$ to $S$ such that the generic fiber $X_{\eta}$ is a smooth curve over $\eta$. Let $X_{s}=X \times_{S} s$ denote the special fiber. Let $l$ be a prime number different from $p$.

Proposition 2.1. Let the notations be as above. We have

$$
\operatorname{dim}_{\mathbb{Q}} G_{1}\left(X_{s}\right)_{\mathbb{Q}}=\operatorname{dim}_{\mathbb{Q}_{\ell}} H_{e ́ t}^{2}\left(X_{\eta}, \mathbb{Q}_{\ell}(2)\right) .
$$

2.1.1. We let $Y=X_{s}^{\text {red }}$ denote the reduced scheme associated with $X_{s}$. It is known that the pushforward map of the canonical closed immersion $Y \hookrightarrow X_{s}$ induces an isomorphism of G-theory. In particular, we have $G_{1}(Y) \stackrel{\cong}{\longrightarrow} G_{1}\left(X_{s}\right)$. Let $Y^{\mathrm{sm}} \stackrel{j}{\hookrightarrow} Y \stackrel{i}{\longleftrightarrow} Y^{\text {sing }}$ denote the smooth locus and the singular locus with the reduced scheme structure of $Y$ respectively. Let $\widetilde{Y} \stackrel{g}{\rightarrow} Y$ denote the normalization. We put $\widetilde{Y^{\mathrm{sm}}}=Y^{\mathrm{sm}} \times_{Y} \widetilde{Y}$ and $\widetilde{Y^{\text {sing }}}=Y^{\text {sing }} \times_{Y} \widetilde{Y}$. 
We have a commutative diagram

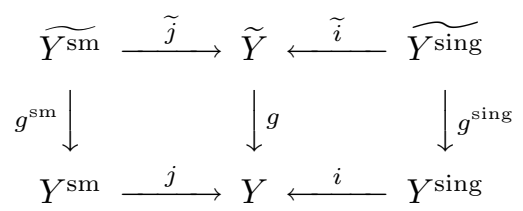

where the two squares are cartesian. Note that $g^{\mathrm{sm}}$ is an isomorphism and that $\tilde{Y}$ is a (not necessarily connected) smooth curve.

2.1.2. We obtain a commutative diagram:

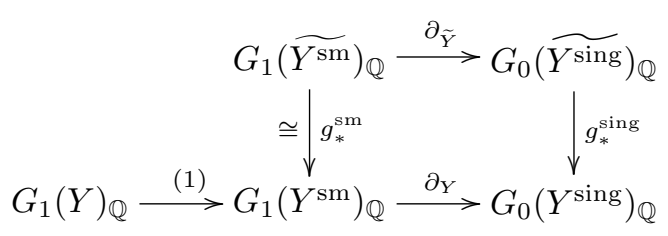

where $\partial_{\widetilde{Y}}$ and $\partial_{Y}$ are the boundary maps in the localization sequence. The restriction map (1) is injective since the $G_{1}$ of a finite field is torsion. We will compute the dimension of the kernel of $\partial_{Y}$ by comparing with étale Borel-Moore homology.

2.1.3. Let $f_{Y}: Y \rightarrow s$ and $f_{\widetilde{Y}}: \widetilde{Y} \rightarrow s$ denote the structure morphisms.

Let $\ell$ be a prime number prime to $p$. Let $\mathcal{F}$ be a constructible étale $\mathbb{Q}_{\ell}$-sheaf on $s$. In the derived category of complexes of étale $\mathbb{Q}_{\ell}$-sheaves with bounded constructible cohomology on $Y$ and on $\tilde{Y}$, we have distinguished triangles $i_{*} i^{!} f_{Y}^{!} \mathcal{F} \rightarrow f_{Y}^{!} \mathcal{F} \rightarrow$ $j_{*} j^{*} f_{Y}^{!} \mathcal{F} \rightarrow$, and $\widetilde{i}_{*} \widetilde{i}^{!} f_{\widetilde{Y}}^{!} \mathcal{F} \rightarrow f_{\widetilde{Y}}^{!} \mathcal{F} \rightarrow \widetilde{j}_{*} \widetilde{j}^{*} f_{\widetilde{Y}}^{!} \mathcal{F} \rightarrow$. Consider the following diagram:

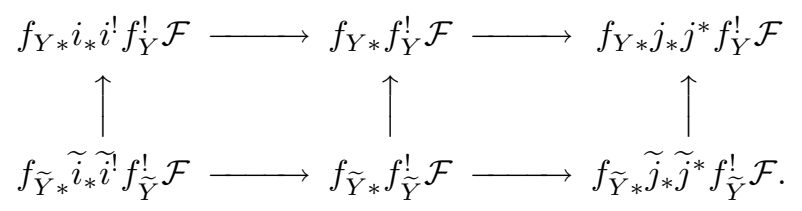

The rows are obtained from the triangles above by applying $f_{Y *}$ and $f_{\widetilde{Y}^{*}}$ respectively. Using base change, we see that $f_{\widetilde{Y} *} \widetilde{i}_{*} \widetilde{i}^{!} f_{\widetilde{Y}}^{!}=f_{Y *} i_{*} i^{!} g_{*} g^{!} f_{Y}^{!}$and $f_{\widetilde{Y} *} \widetilde{j}_{*} \widetilde{j}^{*} f_{\widetilde{Y}}^{!}=$ $f_{Y *} j_{*} j^{*} g_{*} g^{!} f_{Y}^{!}$. We define the vertical arrows to be those induced from the trace $g_{*} g^{!} \rightarrow \mathrm{Id}$, so the diagram is commutative.

We define the Borel-Moore étale homology group of a scheme $X$ of finite type over $s$ to be $H_{a}^{\mathrm{BM}}(X, \mathcal{F})=\mathbb{H}_{\text {ét }}^{-a}\left(X, f_{X}^{!} \mathcal{F}\right)$ where $f_{X}$ is the structure morphism and $\mathcal{F}$ is a constructible étale sheaf of $\mathbb{Z} / l^{n}$-modules on $s$ (see [Ja-Sa, p.488, Example $2.2(\mathrm{a})]$ ). As the rows of the diagram above induce the localization sequence and the vertical arrows induce the pushforward maps (see loc. cit.), we obtain the following commutative diagram:

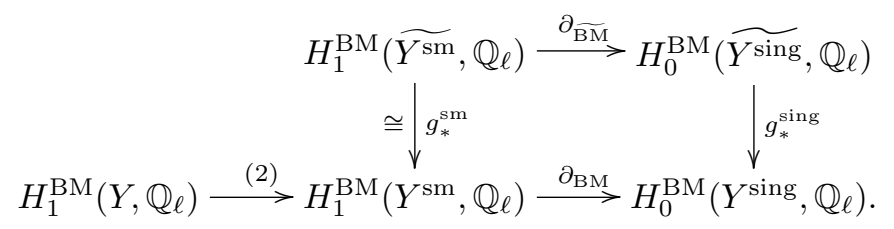

Since $H_{1}^{\mathrm{BM}}\left(Y^{\text {sing }}, \mathbb{Q}_{\ell}\right) \cong H_{\text {ét }}^{1}\left(Y^{\text {sing }}, \mathbb{Q}_{\ell}(1)\right)=0$, the map $(2)$ is injective. 
2.1.4. Now we compare $G$-theory and étale Borel-Moore homology. We consider the following diagram:

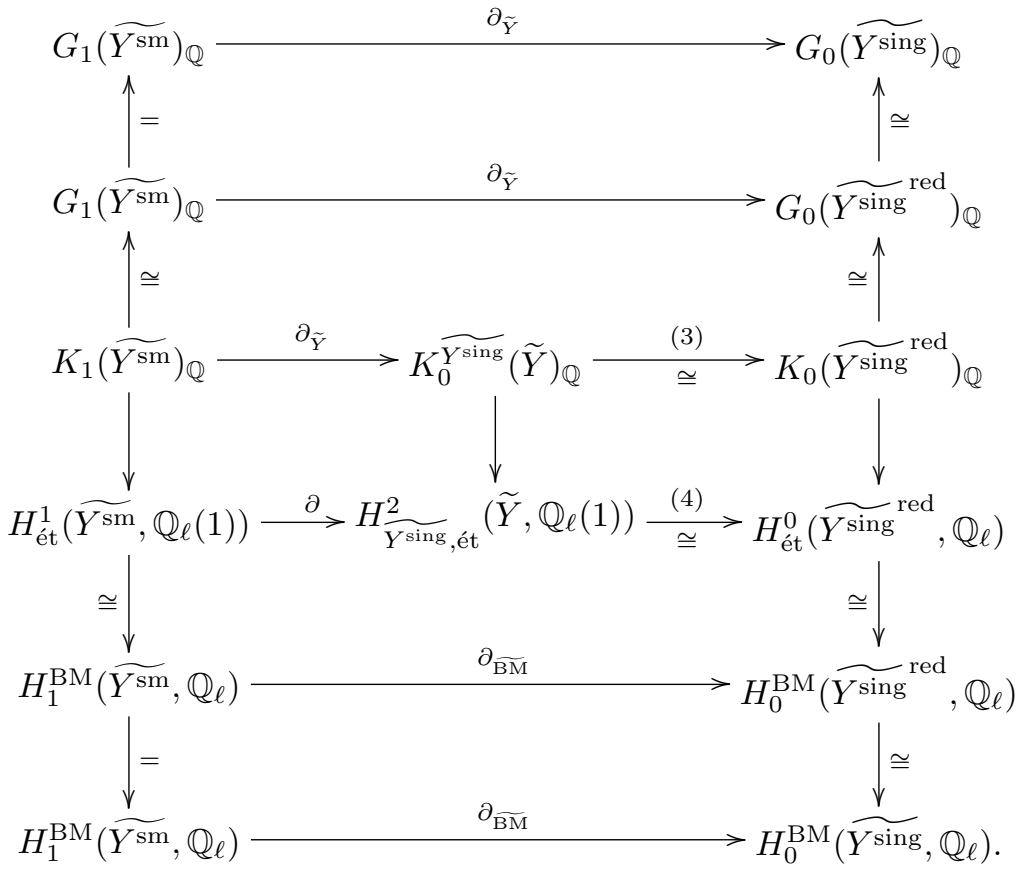

Here we let $\widetilde{Y^{\text {sing }}}$ red denote the reduced scheme associated with $\widetilde{Y^{\text {sing }}}$. Note that it is a reduced zero dimensional scheme hence regular. In the top commutative rectangle, the right vertical arrow is the pushforward by the canonical closed immersion.

In the second rectangle, the vertical maps are the canonical isomorphism of G-groups and K-groups of noetherian regular schemes. The $\partial_{\widetilde{Y}}$ means the boundary map in the respective localization sequence. Here $\widehat{K_{0}^{Y^{\operatorname{sing}}}}(\widetilde{Y})$ is K-theory with support. The rectangle is known to be commutative.

In the third rectangle, the entries of the bottom row (the fourth row of the whole diagram) are the étale cohomology and étale cohomology with support. The three vertical arrows are the Chern class maps and the maps (3) and (4) are the isomomorphisms in [Gi, p.235, Theorem 3.1]. It is known that both squares are commutative ([Gi] loc. cit.). We also know that the first and the third vertical arrows induce isomorphisms when the sources are tensored by $\mathbb{Q}_{\ell}$.

In the fourth rectangle, the vertical maps are the canonical maps induced from $f_{\widetilde{Y^{\mathrm{sm}}}}^{!}=f_{\widetilde{Y^{\mathrm{sm}}}}^{*}[2](1)$ and $f_{Y^{\text {sing }}}^{!}=f_{\widetilde{Y^{\text {sing }}}}^{*}$. The horizontal arrow $\partial_{\widetilde{\mathrm{BM}}}$ is the boundary map. The rectangle is commutative.

In the fifth rectangle, the right vertical arrow is the morphism

$$
\begin{aligned}
& f_{\widetilde{Y^{\text {sing }}}}^{\text {red }} f_{\overparen{Y^{\text {sing }}}}^{!} \mathbb{Q}_{\ell}=f_{\widetilde{Y^{\text {sing }} *}} r_{*} r^{!} f_{\widetilde{Y^{\text {sing }}}} \mathbb{Q}_{\ell} \\
& =D\left(f_{\widetilde{Y^{\text {sing }} *}} r_{*} r^{*} f_{\widetilde{Y^{\text {sing }}}} \mathbb{Q}_{\ell}\right) \rightarrow D\left(f_{\widetilde{Y^{\text {sing }} *}} f_{\widetilde{Y^{\text {sing }}}} \mathbb{Q}_{\ell}\right)=f_{\widetilde{Y^{\text {sing }} *}} f_{\widetilde{Y^{\text {sing }}}}^{!} \mathbb{Q}_{\ell}
\end{aligned}
$$

induced by the adjunction id $\rightarrow r_{*} r^{*}$. Here $D$ denotes the duality. It follows from [SGA4-2, p.367, Theorem 1.1] that the morphism is an isomorphism. 
Some diagram chasing now yields

$$
\operatorname{dim}_{\mathbb{Q}} G_{1}(Y)_{\mathbb{Q}}=\operatorname{dim}_{\mathbb{Q}_{\ell}} H_{1}^{\mathrm{BM}}\left(Y, \mathbb{Q}_{\ell}\right) .
$$

2.1.5. To compute $H_{1}^{\mathrm{BM}}\left(Y, \mathbb{Q}_{\ell}\right)$, note that we have an isomorphism (see [Ja-Sa, p.489, Example $2.2(\mathrm{e})]) H_{1}^{\mathrm{BM}}\left(Y, \mathbb{Q}_{\ell}\right) \cong H_{Y \text {,ét }}^{3}\left(X, \mathbb{Q}_{\ell}(2)\right)$. We use the exact sequence

$$
\begin{aligned}
& H_{\text {ét }}^{2}\left(X, \mathbb{Q}_{\ell}(2)\right) \rightarrow H_{\text {ét }}^{2}\left(X_{\eta}, \mathbb{Q}_{\ell}(2)\right) \stackrel{\alpha}{\rightarrow} H_{Y, \text { ét }}^{3}\left(X, \mathbb{Q}_{\ell}(2)\right) \\
& \rightarrow H_{\text {ét }}^{3}\left(X, \mathbb{Q}_{\ell}(2)\right)
\end{aligned}
$$

of étale cohomology groups. Note that by proper base change, we have $H_{\text {ét }}^{m}\left(X, \mathbb{Q}_{\ell}(2)\right) \cong$ $H_{\text {ét }}^{m}\left(Y, \mathbb{Q}_{\ell}(2)\right)$. It is zero when $m=2$ or 3 by the weight argument. Hence $\alpha$ is an isomorphism. This completes the proof of Proposition 2.1.

2.2. We give an application of Proposition 2.1. Let $\mathcal{E} \rightarrow C$ and $E$ be as in the setup of Theorem 1.1.

Lemma 2.2. Assume that $E \rightarrow$ Spec $k$ has a section, so that $E$ is an elliptic curve. Let $S \subset C$ denote the set of places of $k$ at which $E$ has split multiplicative reduction. Then we have

$$
\operatorname{dim}_{\mathbb{Q}} G_{1}\left(\mathcal{E}_{\wp}\right)_{\mathbb{Q}}= \begin{cases}1 & \text { if } \wp \in S, \\ 0 & \text { if } \wp \notin S .\end{cases}
$$

Proof. Observe that using Proposition 2.1, the left hand side depends only on the generic fiber, which means that it is independent of the choice of the model. Suppose $\mathcal{E}$ is a minimal model. The reduction of a minimal model is classified (see [Liu, p.484, 10.2.1]). One can compute directly the Borel-Moore étale homology (or the G-theory) and see that the claim of the lemma holds true.

\section{FROM SPECIAL FIBER TO GENERIC FIBER}

The aim of this section is to prove Proposition 3.1 below. It compares the boundary map which appeared in the statement of Theorem 1.1 with the Chern class map. This result will be used only for the proof of Theorem 1.1 in Section 11.

3.1. Let the notations (such as $X, S, s$, etc.) be as in Section 2. We let $Y$ denote the complement $X \backslash X_{\eta}$ with the reduced scheme structure. We consider the boundary map

$$
\partial_{\mathbb{Q}}: K_{2}\left(X_{\eta}\right)_{\mathbb{Q}} \rightarrow G_{1}(Y)_{\mathbb{Q}}
$$

supplied by the localization sequence [Qu, p. 127, Proposition 3.2] of the $G$-theory for the pair $(X, Y)$. We fix a prime number $\ell$ different from $p$. We consider the étale Chern class map

$$
c_{2,2, \mathbb{Q}}: K_{2}\left(X_{\eta}\right)_{\mathbb{Q}} \rightarrow H_{\text {ét }}^{2}\left(X_{\eta}, \mathbb{Q}_{\ell}(2)\right),
$$

(or more precisely, the limit of étale Chern class maps with finite coefficients) introduced in [Gi, p. 228-229, Definition 2.22].

Proposition 3.1. For every element $x \in K_{2}\left(X_{\eta}\right)_{\mathbb{Q}}$, we have $\partial_{\mathbb{Q}}(x)=0$ if and only if $c_{2,2, \mathbb{Q}}(x)=0$.

We give two lemmas then the proof. 
3.2. Let $R$ denote the ring of global sections of the sheaf of total quotient rings of $\mathcal{O}_{Y}$. Let $j: G_{1}(Y)_{\mathbb{Q}} \rightarrow G_{1}(R)_{\mathbb{Q}}=\left(R^{\times}\right)_{\mathbb{Q}}$ denote the canonical map. Let $c_{1,1, \mathbb{Q}}:\left(R^{\times}\right)_{\mathbb{Q}} \rightarrow H_{\text {ét }}^{1}\left(\operatorname{Spec} R, \mathbb{Q}_{\ell}(1)\right)$ be the homomorphism supplied by the Kummer theory

$$
R^{\times} /\left(R^{\times}\right)^{\ell^{n}} \cong H_{\text {ét }}^{1}\left(\operatorname{Spec} R, \mathbb{Z} / \ell^{n}(1)\right)
$$

Lemma 3.2. For an element $x \in K_{2}\left(X_{\eta}\right)_{\mathbb{Q}}$, we have $\partial_{\mathbb{Q}}(x)=0$ if and only if $c_{1,1, \mathbb{Q}} \circ j \circ \partial_{\mathbb{Q}}(x)=0$.

Proof. Note that, since the residue field of a closed point $Y$ is a finite field, the group $G_{1}(y)$ is finite for each closed point $y$ of $Y$. This implies that the canonical homomorphism $j: G_{1}(Y)_{\mathbb{Q}} \rightarrow G_{1}(R)_{\mathbb{Q}}=\left(R^{\times}\right)_{\mathbb{Q}}$ is injective.

Let $\widetilde{Y}$ be the normalization of $Y$, and let $\operatorname{Div}(\widetilde{Y})$ denote the group of divisors on $\tilde{Y}$. Since the homomorphism div $: R^{\times} \rightarrow \operatorname{Div}(\tilde{Y})$, which sends $f \in R^{\times}$to the principal divisor $\operatorname{div}(f)$, has a finite kernel and the group $\operatorname{Div}(\tilde{Y})$ is a free abelian group, the intersection $\bigcap_{n}\left(R^{\times}\right)^{\ell^{n}}$ must be finite. Hence the homomorphism $c_{1,1, \mathbb{Q}}$ is injective. The claim is proved.

3.3. Let us consider the exact sequence

$$
H_{\text {ét }}^{2}\left(X, \mathbb{Q}_{\ell}(2)\right) \rightarrow H_{\text {ét }}^{2}\left(X_{\eta}, \mathbb{Q}_{\ell}(2)\right) \stackrel{\partial_{\text {ét }}^{\longrightarrow}}{\longrightarrow} H_{Y, \text { ét }}^{3}\left(X, \mathbb{Q}_{\ell}(2)\right) .
$$

Let $Y^{\text {sing }}$ denote the singular locus of $Y$ equipped with the reduced scheme structure. Let $j_{\text {ét }}: H_{Y \text {,ét }}^{3}\left(X, \mathbb{Q}_{\ell}(2)\right) \rightarrow H_{\text {ét }}^{1}\left(\operatorname{Spec} R, \mathbb{Q}_{\ell}(1)\right)$ denote the composite $H_{Y \text {,ét }}^{3}\left(X, \mathbb{Q}_{\ell}(2)\right) \rightarrow$ $H_{Y \backslash Y^{\text {sing }}}^{3}\left(X \backslash Y^{\text {sing }}, \mathbb{Q}_{\ell}(2)\right) \rightarrow H_{\text {ét }}^{1}\left(Y \backslash Y^{\text {sing }}, \mathbb{Q}_{\ell}(1)\right) \rightarrow H_{\text {ét }}^{1}\left(\operatorname{Spec} R, \mathbb{Q}_{\ell}(1)\right)$, where the first map is the pullback map, the second map is the isomorphism in [Gi, p.235, Theorem 3.1], and the third map is the pullback map.

Lemma 3.3. For an element $x \in K_{2}\left(X_{\eta}\right)_{\mathbb{Q}}$, we have $c_{2,2, \mathbb{Q}}(x)=0$ if and only if $j_{\text {ét }} \circ \partial_{\text {ét }} \circ c_{2,2, \mathbb{Q}}(x)=0$.

Proof. By [SGA4-3, XII, Corollaire 5.5, p. 90], we have $H_{\text {ét }}^{2}\left(X, \mathbb{Q}_{\ell}(2)\right) \cong H_{\text {ét }}^{2}\left(X \times_{S}\right.$ $\left.s, \mathbb{Q}_{\ell}(2)\right) \cong H_{\text {ét }}^{2}\left(Y, \mathbb{Q}_{\ell}(2)\right)$. The weight argument shows that the group $H_{\text {ét }}^{2}\left(Y, \mathbb{Q}_{\ell}(2)\right)$ is zero. Hence the homomorphism $\partial_{\text {ét }}$ is injective.

Let $Z \subset Y$ be a closed subscheme of dimension zero. Consider the exact sequence

$$
H_{Z \text {,ét }}^{3}\left(X, \mathbb{Q}_{\ell}(2)\right) \rightarrow H_{Y, \text { ét }}^{3}\left(X, \mathbb{Q}_{\ell}(2)\right) \rightarrow H_{Y \backslash Z \text {,ét }}^{3}\left(X \backslash Z, \mathbb{Q}_{\ell}(2)\right)
$$

Since $X$ is regular, the absolute purity theorem (cf. [Th, Corollary 1.10 (1.14), p.123] and its proof. See [Fu, Introduction, p. 153] for a general statement and references.) implies that the group $H_{Z \text {,ét }}^{3}\left(X, \mathbb{Q}_{\ell}(2)\right)$ is zero.

The group $H_{\text {ét }}^{1}\left(\operatorname{Spec} R, \mathbb{Q}_{\ell}(1)\right)$ is, again by purity, canonically isomorphic to the inductive limit $\lim _{Z} H_{Y \backslash Z \text {,ét }}^{3}\left(X \backslash Z, \mathbb{Q}_{\ell}(2)\right)$ where $Z$ runs over the closed subschemes of $Y$ of dimension zero. It follows that $j_{\text {ét }}$ is injective. This proves the claim.

3.4. Proof of Proposition 3.1. Let $K$ denote the ring of global sections of the sheaf of total quotient rings of $\mathcal{O}_{X}$. Let $j^{\prime}: K_{2}\left(X_{\eta}\right)_{\mathbb{Q}} \rightarrow K_{2}(K)_{\mathbb{Q}}$ be the pullback 
homomorphism. We have the following commutative diagram

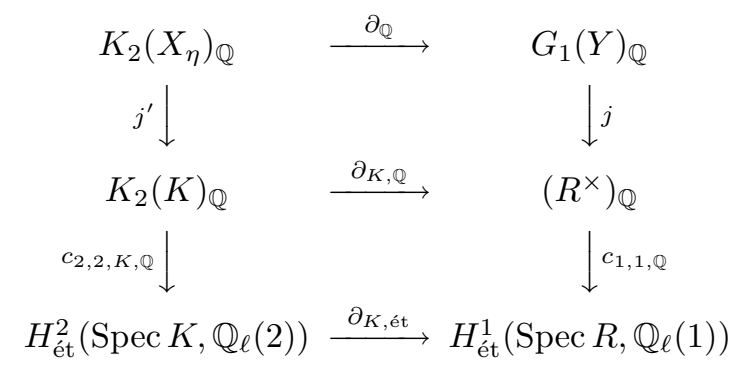

where the maps $\partial_{K, \mathbb{Q}}$ and $\partial_{K \text {,ét }}$ are (the limit of) the maps in the localization sequence of $G$-theory and étale cohomology respectively.

Let $j_{\text {ét }}^{\prime}: H_{\text {ét }}^{2}\left(X_{\eta}, \mathbb{Q}_{\ell}(2)\right) \rightarrow H_{\text {ét }}^{2}\left(\operatorname{Spec} K, \mathbb{Q}_{\ell}(2)\right)$ be the pullback homomorphism. Then the diagram

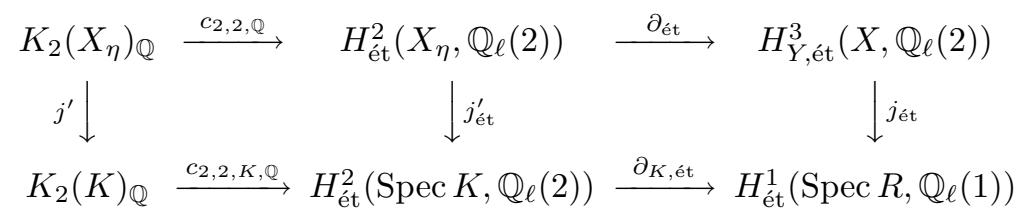

is commutative. From the two commutative diagrams above, we obtain the equalities

$$
c_{1,1, \mathbb{Q}} \circ j \circ \partial_{\mathbb{Q}}=\partial_{K, \text { ét }} \circ c_{2,2, K, \mathbb{Q}} \circ j^{\prime}=j_{\text {ét }} \circ \partial_{\text {ét }} \circ c_{2,2, \mathbb{Q}}
$$

of homomorphisms from $K_{2}\left(X_{\eta}\right)_{\mathbb{Q}}$ to $H_{\text {ét }}^{1}\left(\operatorname{Spec} R, \mathbb{Q}_{\ell}(1)\right)$. Using Lemmas 3.2 and 3.3, the claim of Proposition 3.1 is proved.

\section{Elliptic Sheaves AND the DETERMinant MORPhism}

When Katz and Mazur compute the bad reduction of elliptic modular curves in their book [Ka-Ma], they use extensively the Weil pairing ([Ka-Ma, p.87, (2.8)]) associated to an elliptic curve. Actually they use the morphism from the elliptic modular curves to the spectrum of a ring of cyclotomic integers, which is induced by the Weil pairing (see [Ka-Ma, p.281, 9.4.1]).

The aim of this section is to provide an analogue for Drinfeld modular curves. For this, we do not give the actual pairing for Drinfeld modules, but construct what we call the determinant morphism from the moduli of rank $d$ Drinfeld modules to the moduli of rank 1 Drinfeld modules (Section 4.4). The rough idea is to send a Drinfeld module of rank $d$ to its "determinant Drinfeld module", that is, a maximal exterior power. In the case of rank 2, this may be understood as the morphism induced from the Weil pairing. For application, we only need the case of rank 2, but since the exposition does not become simpler even if we restrict to the rank 2 case, we give the construction in the case of general rank.

There are at least two ways of understanding the notion of the determinant of a Drinfeld module. One is to use the theory of $A$-motives of Anderson and the other is to use the theory of elliptic sheaves. The category of $A$-motives, which contains the category of Drinfeld modules, is equipped with a tensor structure. This direction is pursued by van den Heiden [vdHe]. See his introduction for the details.

We use elliptic sheaves in this article. This notion is equivalent to that of Drinfeld modules (see [Dr3], [Bl-St, Theorem 3.2.1, p. 149]). An elliptic sheaf is by definition a vector bundle with some additional structure, and one expects the determinant 
vector bundle to be equipped with the structure of an elliptic sheaf of rank 1 . This is indeed the case (Lemma 4.3) and gives rise to the desired morphism. An advantage of using elliptic sheaves is that they are equipped with certain canonical structure at the infinity prime. This is helpful when giving the adelic description (using the uniformization of the moduli space) of the determinant morphism.

In Section 4.1, we recall the basic properties of Drinfeld modular varieties. Via the equivalence of the category of elliptic sheaves and the category of Drinfeld modules, we regard them as the properties of the moduli of ellitpic sheaves. We recall the definition of elliptic sheaves and its level structures in Section 4.2.1. In section 4.3, we recall the uniformization of Drinfeld modular varieties. For Drinfeld modular varieties, this has been given in the original paper by Drinfeld. For the moduli of elliptic sheaves, the statement is given in [Bl-St, p.167, Theorem 4.3.5]. We give the details. In particular, the explicit construction of the morphism $\alpha_{d}$ (Section 4.3.3) is given. This will be needed in the proof of Proposition 4.5. Section 4.4 is devoted to the construction of the determinant morphism. Using the moduli interpretation, we obatain the morphism only over $C \backslash(\operatorname{Spec}(A / I) \cup\{\infty\})$ (we refer to the text for notation). Then the regularity of the moduli will give a unique extention to the morphism over $C \backslash\{\infty\}$ (Section 4.4.2).

The results of this section will (directly) be used only in Sections 6.1 and 9.3. In Section 6.1, we will need that the field of constants of the modular curve $M_{I}^{2}$ is the coordinate ring of $M_{I}^{1}$. The structure morphism is precisely given by the determinant morphism. In Section 9.4, we use the uniformization of modular varieties (Section 4.3.4, (4.2)).

We mention that the first work in the actual construction of (the analogue of) the Weil pairing is by Hamahata [Ha] for the base ring $A=\mathbb{F}_{q}[t]$ (see below for notation) and for Drinfeld modules of rank two using the theory of $t$-motives. Van den Heiden [vdHe] also constructs the pairing for general $A$ and for Drinfeld modules of general rank using the theory of $A$-motives. We have not pursued if their constructions give a morphism of moduli spaces.

4.1. Setting. Let $C$ be a projective smooth geometrically irreducible curve over a finite field $\mathbb{F}_{q}$ of $q$ elements of characteristic $p$. For a closed point $\wp \in C$, let $\kappa(\wp)$ denote the residue field at $\wp$. We fix a closed point $\infty \in C$. Let $A=$ $H^{0}\left(C \backslash\{\infty\}, \mathcal{O}_{C}\right)$ denote the coordinate ring of $C \backslash\{\infty\}$.

We let $k=\mathbb{F}_{q}(C)$ denote the function field of $C$. For a prime $\wp$ of $k$, we let $k_{\wp}$ denote the completion of $k$ at $\wp$. We regard a prime of $k$ as a closed point of $C$ and vice versa. If $\wp \neq \infty$ we denote by the same symbol $\wp$ the prime ideal of $A$ corresponding to $\wp$. Then the $\wp$-adic completion $A_{\wp}$ of $A$ is equal to the ring of integers of $k_{\wp}$. We let $\mathcal{O}_{\infty}$ denote the ring of integers of $k_{\infty}$.

Let $d$ be a positive integer. Let $I \subset A$ be a non-zero ideal. We let $M_{I}^{d}$ denote the functor which classifies Drinfeld modules of rank $d$ with a Drinfeld level $I$ structure. When $\operatorname{Spec}(A / I)$ contains two or more closed points, it is representable by an affine scheme over Spec $A$ ([Dr1, Proposition 5.3, p. 576]), and we will denote the latter by the same symbol. We will recall the definition of a Drinfeld module and that of a Drinfeld level $I$ structure in Section 5.1. The scheme $M_{I}^{d}$ has the following properties.

Lemma 4.1. Suppose that $\operatorname{Spec}(A / I)$ contains two or more closed points.

(1) The scheme $M_{I}^{d}$ is a regular scheme of pure dimension $d$. 
(2) The structure morphism $M_{I}^{d} \rightarrow \operatorname{Spec} A$ is of finite type, flat, and surjective. It is smooth when restricted to the open subscheme $U_{I}=\operatorname{Spec} A \backslash$ $\operatorname{Spec}(A / I) \subset \operatorname{Spec} A$.

(3) For an ideal $J \subset I$, the canonical "level-lowering" morphism $M_{J}^{d} \rightarrow M_{I}^{d}$ is finite flat.

Proof. All assertions, except the flatness and the surjectivity in (2), are immediate consequences of [Dr1, Proposition 5.3, p 576] and its corollary. The flatness follows from the local description of $M_{I}^{d}$ in [Dr1, Proposition 5.4, p. 576]. The surjectivity follows from [Dr1, §8, Corollary, p 585].

We recall the fundamental properties of the moduli scheme $M_{I}^{1}$. Let $M_{I / k}^{1}=$ $M_{I}^{1} \times \operatorname{Spec} A \operatorname{Spec} k$.

Lemma 4.2. Suppose that $\operatorname{Spec}(A / I)$ contains two or more closed points. The scheme $M_{I / k}^{1}$ over Spec $k$ is isomorphic to the spectrum of a finite abelian extension of $k$ which is completely split at $\infty$ and unramified outside the primes dividing I. The scheme $M_{I}^{1}$ over $\operatorname{Spec} A$ is isomorphic to the integral closure of Spec $A$ in $M_{I / k}^{1}$.

Proof. This follows from [Dr1, $\S 8$, Theorem 1, p. 585] and its proof.

\subsection{Elliptic sheaves.}

4.2.1. We recall the definition of an elliptic sheaf. Let $S$ be a scheme over $C$. Let $d$ be a positive integer. An elliptic sheaf of rank $d$ over $S$ is a sequence $\left(\mathcal{E}_{i}, j_{i}, t_{i}\right)_{i \in \mathbb{Z}}$,

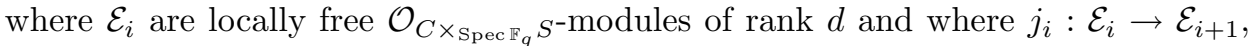
$t_{i}:{ }^{\tau} \mathcal{E}_{i} \rightarrow \mathcal{E}_{i+1}$ are injective $\mathcal{O}_{C \times{ }_{\mathrm{Spec} \mathbb{F}} S}$-linear homomorphisms. Here we put ${ }^{\tau} \mathcal{E}_{i}:=\left(\mathrm{id}_{C} \times \operatorname{Frob}_{S}\right)^{*} \mathcal{E}_{i}$, where Frob ${ }_{S}$ denotes the endomorphism on $S$ which is the identity on the underlying topological space of $S$ and which is the $q$-th power endomorphism on the structure sheaf of $S$. The following conditions should hold.

(1) For each $i$ the following diagram is commutative:

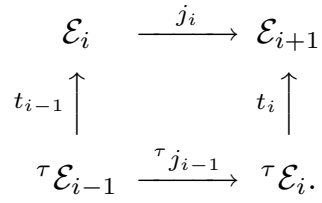

(2) For each $i$ there exists an isomorphism

$$
\mathcal{E}_{i+d \cdot \operatorname{deg}(\infty)} \cong \mathcal{E}_{i}(\infty):=\mathcal{E}_{i} \otimes_{\mathcal{O}_{C \times \mathrm{Spec}_{\mathbb{P}} S}}\left(\mathcal{O}_{C}(\infty) \otimes \mathcal{O}_{S}\right)
$$

where $\mathcal{O}_{C}(\infty) \otimes \mathcal{O}_{S}=\operatorname{pr}_{1}^{*}\left(\mathcal{O}_{C}(\infty)\right) \otimes \operatorname{pr}_{2}^{*}\left(\mathcal{O}_{S}\right)$ is an invertible sheaf on $C \times \times_{\operatorname{Spec} \mathbb{F}_{q}} S$ such that the composite

$$
\mathcal{E}_{i} \hookrightarrow \mathcal{E}_{i+1} \hookrightarrow \cdots \hookrightarrow \mathcal{E}_{i+d \cdot \operatorname{deg}(\infty)} \cong \mathcal{E}_{i}(\infty)
$$

is the canonical embedding.

(3) The direct image of $\mathcal{E}_{i+1} / j_{i}\left(\mathcal{E}_{i}\right)$ under the projection to the second factor $\operatorname{pr}_{S}: C \times_{\operatorname{Spec} \mathbb{F}_{q}} S \rightarrow S$ is a locally free $\mathcal{O}_{S}$-module of rank one.

(4) For each $i \in \mathbb{Z}$, the cokernel Coker $t_{i}$ is supported on the graph of a morphism $\iota_{i}: S \rightarrow C$ over $\operatorname{Spec} \mathbb{F}_{q}$, and is the direct image of a locally free module on $S$ of rank one by the morphism $S \stackrel{\left(\iota_{i}, \mathrm{id}_{S}\right)}{\longrightarrow} C \times \times_{\mathrm{Spec}_{\mathrm{F}}} S$. Moreover, when $i=-d(g-1)$, the morphism $\iota_{-d(g-1)}$ equals the structure morphism $S \rightarrow C$, where $g$ is the genus of the curve $C$. 
(5) For any closed point $s \in S$, one has $\operatorname{deg}\left(\left.\mathcal{E}_{0}\right|_{C \times_{\mathrm{Spec}_{q}}\{s\}}\right)=d(g-1)+1$.

4.2.2. Level structure. Let $I \subset A$ be a nonzero ideal. Let $S$ be a scheme over $C \backslash$ $\operatorname{Spec}(A / I)$. Let $\left(\mathcal{E}_{i}, j_{i}, t_{i}\right)_{i \in \mathbb{Z}}$ be an elliptic sheaf of rank $d$ over $S$. The morphisms

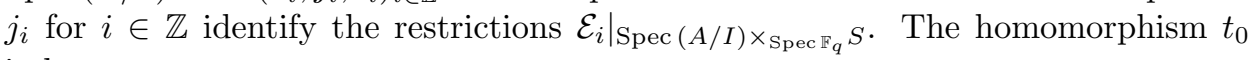
induces

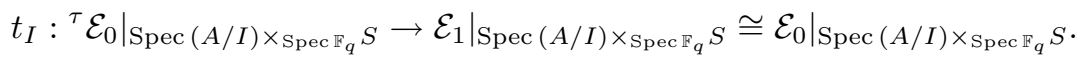

A level $I$ structure on $\left(\mathcal{E}_{i}, j_{i}, t_{i}\right)_{i \in \mathbb{Z}}$ is an isomorphism

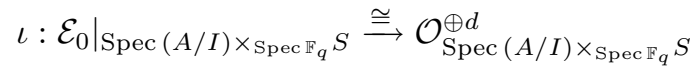

such that the composite

$$
\tau_{\iota}:\left.{ }^{\tau} \mathcal{E}_{0}\right|_{\operatorname{Spec}(A / I) \times_{\mathrm{Spec} \mathbb{q}} S} \stackrel{\cong}{\longrightarrow}\left(\mathcal{O}_{\mathrm{Spec}(A / I) \times_{\mathrm{Spec} \mathbb{q}} S}\right)^{\oplus d}=\mathcal{O}_{\mathrm{Spec}(A / I) \times_{\mathrm{Spec} \mathbb{F}} S}^{\oplus d}
$$

equals $\iota \circ t_{I}$.

If $S$ is a scheme over $\operatorname{Spec} A$, there is a canonical one-to-one correspondence between the isomorphism classes of Drinfeld modules of rank $d$ over $S$ with a Drinfeld level $I$ structure and those of elliptic sheaves of rank $d$ over $S$ with a level $I$ structure ([Dr3], [Bl-St, Theorem 3.2.1, p. 149]).

Let $M_{I, C \backslash \operatorname{Spec}(A / I)}^{d}$ denote the moduli stack over $C \backslash \operatorname{Spec}(A / I)$ of the elliptic sheaves of rank $d$ with a level $I$ structure, introduced and denoted by $\mathcal{E} \ell_{X, I}^{(d)}$ in [Bl-St, Definition 3.1.11, p. 148]. It is an algebraic stack in the sense of DeligneMumford. Using the one-to-one correspondence mentioned in the previous paragraph, we identify the base change $M_{I, C \backslash \operatorname{Spec}(A / I)}^{d} \times_{C \backslash \operatorname{Spec}(A / I)}(\operatorname{Spec} A \backslash \operatorname{Spec}(A / I))$ with the stack $M_{I}^{d} \times_{\operatorname{Spec} A}(\operatorname{Spec} A \backslash \operatorname{Spec}(A / I))$.

4.3. Uniformization of Drinfeld modular varieties. We recall in this section the uniformization of Drinfeld modular varieties at infinity. We give the adelic description using elliptic sheaves. Many of the statements are found in the paper [Bl-St, §4] by Blum and Stuhler; we give the explicit details for later use.

We denote by $\mathbb{A}$ the $k$-algebra of adeles of $k$, by $\mathbb{A}^{\infty}$ the $k$-algebra of finite adeles of $k$, and by $\widehat{A}$ the projective $\operatorname{limit} \lim _{I} A / I$ where $I$ runs over all non-zero ideals of $A$. We recall that $\widehat{A}$ is a subring of $\mathbb{A}^{\infty}$ and $\mathbb{A}^{\infty}=\widehat{A} \otimes_{A} k$. Let $d$ be a positive integer. Let $k_{\infty}$ denote the completion of $k$ at $\infty$, and $\mathcal{O}_{\infty}$ be the ring of integers. We let $\pi_{\infty} \in \mathcal{O}_{\infty}$ denote a uniformizer.

4.3.1. Elliptic sheaves at infinity. We follow [Bl-St, p.163] and define a functor $\widehat{\text { Ell }}^{(d)}$ from Nilp to the category of sets as follows. Here Nilp denotes the category of commutative $\mathcal{O}_{\infty}$-algebras $R$ such that the image of $\pi_{\infty}$ in $R$ is nilpotent.

For an object $R$ in Nilp, we denote by Frob $_{R}$ the $q$-power endomorphism of $R$. For a finitely generated $\mathcal{O}_{\infty}$-module $M$ and an $\mathbb{F}_{q}$-algebra $N$, we let $M \widehat{\otimes}_{\mathbb{F}_{q}} N$ denote the $\left(\pi_{\infty} \otimes 1\right)$-adic completion of $M \otimes_{\mathbb{F}_{q}} N$. For a finite dimensional $k_{\infty}$-vector space $V$ and an $\mathbb{F}_{q}$-algebra $N^{\prime}$, we let $V \widehat{\otimes}_{\mathbb{F}_{q}} N^{\prime}$ denote the inductive limit $\lim _{\Lambda} \Lambda \widehat{\otimes}_{\mathbb{F}_{q}} N^{\prime}$ where $\Lambda$ runs over the $\mathcal{O}_{\infty}$-lattices of $V$.

Let $R$ be an object of Nilp. Then $\widehat{\operatorname{Ell}}^{(d)}(R)$ is the set of isomorphism classes of sequences $\left(\Lambda_{i}, t_{i}\right)_{i \in \mathbb{Z}}$ whose descriptions are given below:

- For each $i \in \mathbb{Z}$, the $\Lambda_{i}$ is an $\mathcal{O}_{\infty} \widehat{\otimes}_{\mathbb{F}_{q}} R$-submodule of $k_{\infty}^{\oplus d} \widehat{\otimes}_{\mathbb{F}_{q}} R$. The $\Lambda_{i}$ 's satisfy 
(1) $\cdots \subset \Lambda_{i-1} \subset \Lambda_{i} \subset \Lambda_{i+1} \subset \cdots$, and

(2) $\Lambda_{i+d \cdot \operatorname{deg}(\infty)}=\left(\pi_{\infty}^{-1} \otimes 1\right) \Lambda_{i}$.

- Each $\Lambda_{i}$ is, as an $\mathcal{O}_{\infty} \widehat{\otimes}_{\mathbb{F}_{q}} R$-module, locally free of rank $d$.

- For each $i$, there exists a morphism $\alpha_{i}$ : Spec $R \rightarrow \operatorname{Spec} \kappa(\infty)$ such that the quotient $\Lambda_{i+1} / \Lambda_{i}$ is supported on the graph of the composite $\operatorname{Spec} R \stackrel{\alpha_{i}}{\longrightarrow}$ $\operatorname{Spec} \kappa(\infty) \rightarrow \operatorname{Spec} \mathcal{O}_{\infty}$ where the second morphism is the canonical closed immersion.

- For each $i$, the quotient $\Lambda_{i} / \Lambda_{i+1}$, regarded as an $R$-module via the canonical homomorphism $R \rightarrow \mathcal{O}_{\infty} \widehat{\otimes}_{\mathbb{F}_{q}} R$ which sends $r \in R$ to $1 \otimes r$, is locally free of rank one.

- For each $i$, the $t_{i}$ is an injective homomorphism ${ }^{\tau} \Lambda_{i} \rightarrow \Lambda_{i+1}$ of $\mathcal{O}_{\infty} \widehat{\otimes}_{\mathbb{F}_{q}} R$ modules. Here for an $\mathcal{O}_{\infty} \widehat{\otimes}_{\mathbb{F}_{q}} R$-module $\Lambda$, we denote by ${ }^{\tau} \Lambda$ the base change

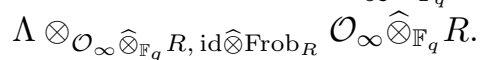

- For each $i$, the diagram

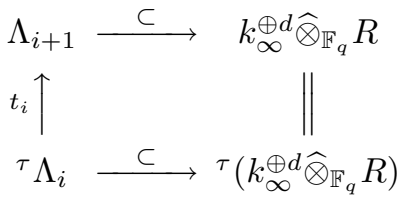

is commutative.

- For each $i$, the cokernel of $t_{i}$ is supported on the graph of a morphism $\operatorname{Spec} R \rightarrow \operatorname{Spec} \mathcal{O}_{\infty}$ over $\operatorname{Spec} \mathbb{F}_{q}$. Moreover, when $i=-d(g-1)$, this homomorphism equals the structure morphism $\operatorname{Spec} R \rightarrow \operatorname{Spec} \mathcal{O}_{\infty}$. Here $g$ denotes the genus of $C$.

- Each Coker $t_{i}$, regarded as an $R$-module via the canonical homomorphism $R \rightarrow \mathcal{O}_{\infty} \widehat{\otimes}_{\mathbb{F}_{q}} R$ which sends $r \in R$ to $1 \otimes r$, is locally free of rank one.

4.3.2. Let $I \subset A$ be a non-zero ideal. Let $\mathbb{K}_{I}$ denote the compact open subgroup $\operatorname{Ker}\left[\mathrm{GL}_{d}(\widehat{A}) \rightarrow \mathrm{GL}_{d}(A / I)\right]$ of $\mathrm{GL}_{d}\left(\mathbb{A}^{\infty}\right)$. For a formal scheme $F$ over Spf $\mathcal{O}_{\infty}$ on which the group $\mathrm{GL}_{d}(k)$ acts from the left, we define the formal stack

$$
\mathrm{GL}_{d}(k) \backslash\left(\mathrm{GL}_{d}\left(\mathbb{A}^{\infty}\right) / \mathbb{K}_{I} \times F\right)
$$

as the quotient of the product $\left(\coprod_{\mathrm{GL}_{d}\left(\mathbb{A}^{\infty}\right) / \mathbb{K}_{I}} \operatorname{Spf} \mathcal{O}_{\infty}\right) \times_{\operatorname{Spf} \mathcal{O}_{\infty}} F$ by the diagonal action of $\mathrm{GL}_{d}(k)$. Here $\mathrm{GL}_{d}(k)$ acts on $\coprod_{\mathrm{GL}_{d}\left(\mathbb{A}^{\infty}\right) / \mathbb{K}_{I}}$ Spf $\mathcal{O}_{\infty}$ from the left on the index set.

If we regard $F$ as a covariant functor from Nilp to the category of sets, then the formal stack $\mathrm{GL}_{d}(k) \backslash\left(\mathrm{GL}_{d}\left(\mathbb{A}^{\infty}\right) / \mathbb{K}_{I} \times F\right)$ is the category fibered in groupoids over the category Nilp ${ }^{\text {op }}$ whose description is given as follows:

- An object in $\mathrm{GL}_{d}(k) \backslash\left(\mathrm{GL}_{d}\left(\mathbb{A}^{\infty}\right) / \mathbb{K}_{I} \times F\right)$ is a triple $(R, Q, \alpha)$ which consists of an object $R$ in Nilp, a left $\mathrm{GL}_{d}(k)$-torsor $Q$ over $\operatorname{Spec} R$, and a $\mathrm{GL}_{d}(k)$-equivariant morphism $\alpha: Q \rightarrow \mathrm{GL}_{d}\left(\mathbb{A}^{\infty}\right) / \mathbb{K}_{I} \times\left(F \times \operatorname{Spf} \mathcal{O}_{\infty} \operatorname{Spec} R\right)$. Here $F \times_{\text {Spf } \mathcal{O}_{\infty}}$ Spec $R$ denote the product of $F$ and the covariant functor represented by $R$ from Nilp to the category of sets, which we regard as a scheme over $\operatorname{Spec} R$, and $\mathrm{GL}_{d}\left(\mathbb{A}^{\infty}\right) / \mathbb{K}_{I} \times\left(F \times_{\operatorname{Spf} \mathcal{O}_{\infty}} \operatorname{Spec} R\right)$ denotes the disjoint union of copies of the scheme $F \times_{\operatorname{Spf}} \mathcal{O}_{\infty}$ Spec $R$ indexed by the set $\mathrm{GL}_{d}\left(\mathbb{A}^{\infty}\right) / \mathbb{K}_{I}$ where the group $\mathrm{GL}_{d}(k)$ acts both on the index set $\mathrm{GL}_{d}\left(\mathbb{A}^{\infty}\right) / \mathbb{K}_{I}$ and the scheme $F \times_{\text {Spf } \mathcal{O}_{\infty}} \operatorname{Spec} R$. 
- For two objects $(R, Q, \alpha),\left(R^{\prime}, Q^{\prime}, \alpha^{\prime}\right)$ in $\mathrm{GL}_{d}(k) \backslash\left(\mathrm{GL}_{d}\left(\mathbb{A}^{\infty}\right) / \mathbb{K}_{I} \times F\right)$, the set of morphisms from $(R, Q, \alpha)$ to $\left(R^{\prime}, Q^{\prime}, \alpha^{\prime}\right)$ is the set of pairs $(f, \phi)$ where $f: R^{\prime} \rightarrow R$ is a morphism in Nilp and $\phi: Q^{\prime} \times_{\operatorname{Spec}} R^{\prime} \operatorname{Spec} R \stackrel{\cong}{\longrightarrow} Q$ is an isomorphism of $\mathrm{GL}_{d}(k)$-torsors over $\operatorname{Spec} R$ such that the diagram

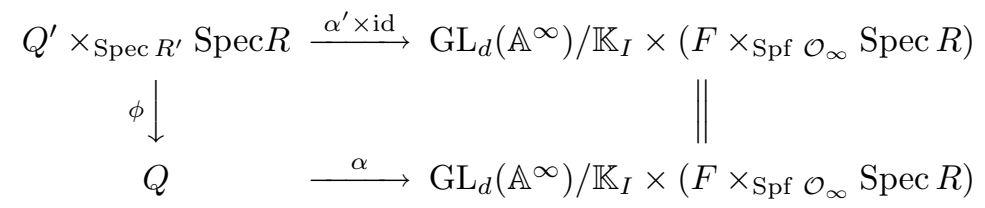

is commutative.

- The functor $\mathrm{GL}_{d}(k) \backslash\left(\mathrm{GL}_{d}\left(\mathbb{A}^{\infty}\right) / \mathbb{K}_{I} \times F\right) \rightarrow$ Nilp ${ }^{\text {op }}$ is given by sending $(R, Q, \alpha)$ to $R$.

For an object $R$ in Nilp, we denote by $\left(\mathrm{GL}_{d}(k) \backslash\left(\mathrm{GL}_{d}\left(\mathbb{A}^{\infty}\right) / \mathbb{K}_{I} \times F\right)\right)(R)$ the fiber of $\mathrm{GL}_{d}(k) \backslash\left(\mathrm{GL}_{d}\left(\mathbb{A}^{\infty}\right) / \mathbb{K}_{I} \times F\right)$ over $R$. We will use the notation introduced in this paragraph only for $F=\widehat{\operatorname{Ell}}^{(d)}$ and for $F=\widehat{\Omega}^{(d)}$, where the formal scheme $\widehat{\Omega}^{(d)}$ will appear in Section 4.3.4. In both cases, $F$ admits a left action of the group $\mathrm{GL}_{d}\left(k_{\infty}\right)$, and hence of $\mathrm{GL}_{d}(k)$.

4.3.3. Let $\widehat{M}_{I, \infty}^{d}$ denote the formal completion of the algebraic stack $M_{I, C \backslash \operatorname{Spec}(A / I)}^{d}$ along its special fiber $M_{I, \infty}^{d}$ at $\infty \in C$ (see [Bl-St, Theorem 4.3.5, p. 167]). We define a morphism $\alpha_{d}: \widehat{M}_{I, \infty}^{d} \rightarrow \mathrm{GL}_{d}(k) \backslash\left(\mathrm{GL}_{d}\left(\mathbb{A}^{\infty}\right) / \mathbb{K}_{I} \times \widehat{\mathrm{Ell}}^{(d)}\right)$ of formal stacks.

We use the following notation. For a projective $A$-module $P$ of finite rank, we denote by $\widetilde{P}$ the locally free $\mathcal{O}_{\text {Spec } A}$-module of finite rank corresponding to $P$. For two schemes $X, S$ over Spec $\mathbb{F}_{q}$, we denote by $X \times S$ the fiber product $X \times_{\text {Spec } \mathbb{F}_{q}} S$. For an $\mathcal{O}_{X}$-module $\mathcal{F}$, we denote by $\mathcal{F} \bigotimes_{\mathbb{F}_{q}} \mathcal{O}_{S}$ the pullback of $\mathcal{F}$ under the first projection $X \times S \rightarrow X$.

Let $R$ be an object in Nilp. We construct a map of $R$-valued points. Let $\mathcal{E}=\left(\mathcal{E}_{i}, j_{i}, t_{i}\right)_{i \in \mathbb{Z}}$ be an elliptic sheaf over $S=\operatorname{Spec} R$ with a level $I$ structure. The restriction $\left.\mathcal{E}_{i}\right|_{(\operatorname{Spec} A) \times S}$ does not depend on $i$ in the sense that the injection $j_{i}: \mathcal{E}_{i} \hookrightarrow \mathcal{E}_{i+1}$ induces an isomorphism $\left.\left.\mathcal{E}_{i}\right|_{(\operatorname{Spec} A) \times S} \cong \mathcal{E}_{i+1}\right|_{(\operatorname{Spec} A) \times S}$. By [St, $\S 3$, Proposition1, p. 508], there exists a projective $A$-module $P=P_{\mathcal{E}}$ of rank $d$ such that the contravariant functor $Q_{\mathcal{E}}^{\prime}$ from the category of schemes over $S$ to the category of sets which associates, to a scheme $T$ over $S$, the set of isomorphisms

$$
\psi:\left.\mathcal{E}_{i+1}\right|_{(\operatorname{Spec} A) \times T} \cong \widetilde{P} \bigotimes_{\mathbb{F}_{q}} \mathcal{O}_{T}
$$

of $\mathcal{O}_{(\operatorname{Spec} A) \times T}$-modules such that the diagram

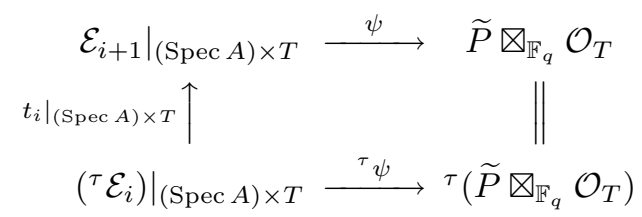

is commutative, is representable by an étale left $\operatorname{Aut}_{A}(P)$-torsor over $S$. Here the symbol $\left.\right|_{(\operatorname{Spec} A) \times T}$ denotes the pullback to $(\operatorname{Spec} A) \times T$.

We fix an isomorphism $\mu=\mu_{\mathcal{E}}: P \otimes_{A} k \stackrel{\cong}{\rightrightarrows} k^{\oplus d}$. We regard the group $\operatorname{Aut}_{A}(P)$ as a subgroup of $\mathrm{GL}_{d}(k)$ via this isomorphism. We define the left $\mathrm{GL}_{d}(k)$-torsor $Q_{\mathcal{E}}=Q_{\mathcal{E}, \mu}$ over $S$ as $Q_{\mathcal{E}}=\mathrm{GL}_{d}(k) \times_{\operatorname{Aut}_{A}(P)} Q_{\mathcal{E}}^{\prime}$. 
Let $T=\operatorname{Spec} R^{\prime}$ be a connected affine scheme over $S$ and let $\psi \in Q_{\mathcal{E}}^{\prime}(T)$. (Note that $R^{\prime}$ is then an object in Nilp.) For each $i \in \mathbb{Z}$, let us consider the completion of the pullback of $\mathcal{E}_{i}$ to $C \times T$ along Spec $\kappa(\infty) \times T$. Via the isomorphism $\psi$, this completion gives an $\mathcal{O}_{\infty} \widehat{\otimes}_{\mathbb{F}_{q}} R^{\prime}$-lattice $\Lambda_{\psi, i}^{\prime}$ in $\left(P \otimes_{A} k_{\infty}\right) \widehat{\otimes}_{\mathbb{F}_{q}} R^{\prime}$. We denote by $\Lambda_{\psi, i}$ the image of $\Lambda_{\psi, i}^{\prime}$ under the isomorphism $(\mu \otimes \mathrm{id}) \widehat{\otimes} \mathrm{id}:\left(P \otimes_{A} k_{\infty}\right) \widehat{\otimes}_{\mathbb{F}_{q}} R^{\prime}=$ $\left(\left(P \otimes_{A} k\right) \otimes_{k} k_{\infty}\right) \widehat{\otimes}_{\mathbb{F}_{q}} R^{\prime} \cong\left(k^{\oplus d} \otimes_{k} k_{\infty}\right) \widehat{\otimes}_{\mathbb{F}_{q}} R^{\prime}=k_{\infty}^{\oplus d} \widehat{\otimes}_{\mathbb{F}_{q}} R^{\prime}$. The homomorphisms $j_{i}: \mathcal{E}_{i} \rightarrow \mathcal{E}_{i+1}$ and $t_{i}:{ }^{\tau} \mathcal{E}_{i} \rightarrow \mathcal{E}_{i+1}$ then induce homomorphisms $j_{\psi, i}: \Lambda_{\psi, i} \rightarrow \Lambda_{\psi, i+1}$ and $t_{\psi, i}:{ }^{\tau} \Lambda_{\psi, i} \rightarrow \Lambda_{\psi, i+1}$. These data define an element $\left(\Lambda_{\psi, i}, t_{\psi, i}\right)_{i \in \mathbb{Z}}$ in $\widehat{\operatorname{Ell}}^{(d)}\left(R^{\prime}\right)$. Suppose that the level $I$ structure on the elliptic sheaf $\left(\mathcal{E}_{i}, t_{i}, j_{i}\right)_{i \in \mathbb{Z}}$ is given. Via the isomorphism $\psi$, this level structure gives an isomorphism $\left.\widetilde{P} \otimes_{\mathbb{F}_{q}} \mathcal{O}_{T}\right|_{\operatorname{Spec}(A / I) \times T} \cong$ $\mathcal{O}_{\text {Spec }(A / I) \times T}^{\oplus d}$. Since $T$ is connected, to give such an isomorphism is equivalent to giving an isomorphism $\iota_{\psi}: P / I P \cong(A / I)^{\oplus d}$ of $A$-modules.

For each prime $\wp \neq \infty$ of $k$, take an isomorphism $\widetilde{\iota}_{\psi, \wp}: A_{\wp}^{\oplus d} \stackrel{\cong}{\longrightarrow} P \otimes_{A} A_{\wp}$ such that the diagram

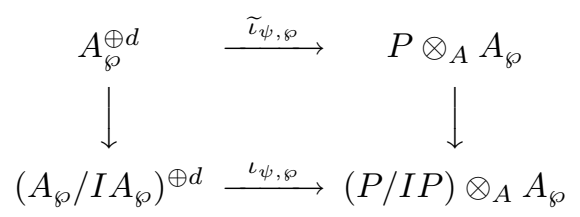

is commutative, where the vertical homomorphisms are the natural projections and the isomorphism $\iota_{\psi, \wp}$ is the one induced by the isomorphism $\iota_{\psi}$. Let $g_{\psi, \wp}$ be the $d \times d$ matrix with entries in $k_{\wp}$ such that the composite

$k_{\wp}^{\oplus d}=A_{\wp}^{\oplus d} \otimes_{A_{\wp}} k_{\wp} \stackrel{\tau_{\psi, \wp} \otimes \mathrm{id}}{\longrightarrow} P \otimes_{A} A_{\wp} \otimes_{A_{\wp}} k_{\wp}=P \otimes_{A} k \otimes_{k} k_{\wp} \stackrel{\mu \otimes \mathrm{id}}{\longrightarrow} k^{\oplus d} \otimes_{k} k_{\wp}=k_{\wp}^{\oplus d}$

is supplied by the right multiplication by $g_{\psi, \wp}$ (here we regard $k_{\wp}^{\oplus d}$ as the space of row vectors). Then class of the element $\left(g_{\psi, \wp}\right)_{\wp \neq \infty} \in \mathrm{GL}_{d}\left(\mathbb{A}^{\infty}\right)$ in $\mathrm{GL}_{d}\left(\mathbb{A}^{\infty}\right) / \mathbb{K}_{I}$ does not depend on the choice of $\widetilde{\iota}_{\psi, \wp}$.

We define the $\operatorname{Aut}_{A}(P)$-equivariant morphism $\alpha_{\mathcal{E}}^{\prime}: Q_{\mathcal{E}}^{\prime} \rightarrow \mathrm{GL}_{d}\left(\mathbb{A}^{\infty}\right) / \mathbb{K}_{I} \times$ $\left(\widehat{\mathrm{Ell}}^{(d)} \times \operatorname{Spec} R\right)$ as the morphism which send $\psi \in Q_{\mathcal{E}}^{\prime}(T)$ for each connected affine scheme $T$ over $S$ to $\left(\left(g_{\psi, \wp}\right)_{\wp \neq \neq},\left(\Lambda_{\psi, i}, t_{\psi, i}\right)_{i \in \mathbb{Z}}\right)$. The morphism $\alpha_{\mathcal{E}}^{\prime}$ induces a $\mathrm{GL}_{d}(k)$-equivariant morphism $\alpha_{\mathcal{E}}: Q_{\mathcal{E}} \rightarrow \mathrm{GL}_{d}\left(\mathbb{A}^{\infty}\right) / \mathbb{K}_{I} \times\left(\widehat{\operatorname{Ell}}^{(d)} \times \operatorname{Spec} R\right)$ which does not depend on the choice in the sense that for two isomorphisms $\mu_{1}, \mu_{2}: P \otimes_{A} k \stackrel{\cong}{\rightrightarrows} k^{\oplus d}$, there exists a canonical isomorphism $\nu: Q_{\mathcal{E}, \mu_{1}} \cong Q_{\mathcal{E}, \mu_{2}}$ of left $\mathrm{GL}_{d}(k)$-torsors such that the diagram

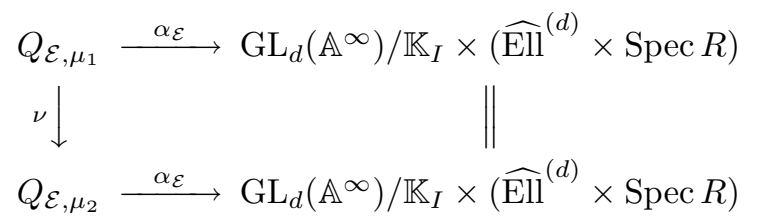

is commutative. We define the morphism $\alpha_{d}$ as the morphism induced by the functor

$$
\widehat{M}_{I, \infty}^{d}(R) \rightarrow\left(\mathrm{GL}_{d}(k) \backslash\left(\mathrm{GL}_{d}\left(\mathbb{A}^{\infty}\right) / \mathbb{K}_{I} \times \widehat{\mathrm{Ell}}^{(d)}\right)(R)\right.
$$

which sends $\left(\mathcal{E}_{i}, t_{i}, j_{i}\right)_{i \in \mathbb{Z}}$ to $\left(Q_{\mathcal{E}}, \alpha_{\mathcal{E}}\right)$ for each object $R$ in Nilp.

Using the argument in the proof of [St, §3, Theorem 2, p. 514] (where the level structure is not considered), one can prove that $\alpha_{d}$ is an isomorphism. 
4.3.4. Let $\mathfrak{X}_{d}$ be the Drinfeld symmetric space (see [Bl-St, 4.2, p. 162] where it is denoted by $\left.\Omega_{\text {rig }}^{(d)}\right)$ for $\mathrm{GL}_{d}$. It is a rigid analytic space over $k_{\infty}$, and the group $\mathrm{GL}_{d}\left(k_{\infty}\right)$ acts from the left on $\mathfrak{X}_{d}$. When $d=1$, the space $\mathfrak{X}_{1}$ is just one point. The rigid analytic space $\mathfrak{X}_{d}$ has a canonical formal model $\widehat{\Omega}^{(d)}$ over $\mathcal{O}_{\infty}$, which is constructed by Deligne and whose definition is given in [Gen, III, Définition 1.2.2, p. 73] (where it is denoted by $\widehat{\Omega}^{d}$ ).

One can verify that the functor $\widehat{\mathrm{Ell}}^{(d)}$ and the functor $G_{\mathcal{O}}$ of Genestier ([Gen, III, Définition I.4.3.3, p. 29]) (where their $\mathcal{O}$ is our $\mathcal{O}_{\infty}$ ) are isomorphic by comparing our definition of $\widehat{\mathrm{Ell}}^{(d)}$ in Section 4.3.1 and the definition by Genestier [Gen, Variant 4.4 .2 de la définition 4.3.3, p. 30]. It is proved in [Gen, 3.1, Théorème 1.1, p. 94] that the functors $\widehat{\Omega}^{(d)}$ and $G_{\mathcal{O}}$ are isomorphic. Hence we obtain a uniformization

$$
\widehat{M}_{I, \infty}^{d} \cong \mathrm{GL}_{d}(k) \backslash\left(\mathrm{GL}_{d}\left(\mathbb{A}^{\infty}\right) / \mathbb{K}_{I} \times \widehat{\Omega}^{(d)}\right)
$$

where the isomorphism is given by the composition of the isomorphism $\alpha_{d}$ and the isomorphism between $\widehat{\Omega}^{(d)}$ and $\widehat{\mathrm{Ell}}^{(d)}$ (Recall the convention in Section 4.3.2). Here $\mathrm{GL}_{d}\left(\mathbb{A}^{\infty}\right) / \mathbb{K}_{I} \times \widehat{\Omega}^{(d)}$ denotes the disjoint union of the copies of $\widehat{\Omega}^{(d)}$ indexed by the set $\mathrm{GL}_{d}\left(\mathbb{A}^{\infty}\right) / \mathbb{K}_{I}$, on which the group $\mathrm{GL}_{d}(k)$ acts from the left via its action on $\mathrm{GL}_{d}\left(\mathbb{A}^{\infty}\right) / \mathbb{K}_{I}$ and on $\widehat{\Omega}^{(d)}$.

For a quasi-projective scheme $X$ over Spec $k_{\infty}$, let $X^{\text {an }}$ denote the rigid analytic space associated to $X$. By taking the generic fiber in the sense of Raynaud of both sides of (4.1), one obtains the uniformization of analytic Drinfeld modular varieties (cf. [Dr1], [Bl-St, Remark 4.3.1 ii), p. 166, Theorem 4.3.5, p. 167]):

$$
\left(M_{I / k_{\infty}}^{d}\right)^{\mathrm{an}} \cong \mathrm{GL}_{d}(k) \backslash\left(\mathrm{GL}_{d}\left(\mathbb{A}^{\infty}\right) / \mathbb{K}_{I} \times \mathfrak{X}_{d}\right) .
$$

4.4. The determinant morphism. In this section, we construct what we call a determinant morphism.

4.4.1. Let $I \varsubsetneqq A$ be a non-zero ideal. Let $S$ be a scheme over $C \backslash \operatorname{Spec}(A / I)$. Given an elliptic sheaf $\left(\mathcal{E}_{i}, t_{i}, j_{i}\right)_{i \in \mathbb{Z}}$ over $S$ of rank $d$ with a level $I$ structure $\iota$ : $\left.\mathcal{E}_{0}\right|_{\operatorname{Spec}(A / I) \times_{\text {Spec } \mathbb{F}_{q}} S} \cong \mathcal{O}_{\text {Spec }(A / I) \times_{\text {Spec } \mathbb{F}_{q}} S}^{\oplus d}$, we define its determinant elliptic sheaf to be the triple $\left(\mathcal{F}_{i}, t_{i}^{\prime \prime}, j_{i}^{\prime \prime}\right)$, where $\mathcal{F}_{i}$ is the exterior power $\mathcal{F}_{i}=\wedge^{d} \mathcal{E}_{i-(d-1)(g-1)}$

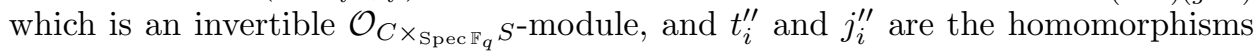
$t_{i}^{\prime \prime}=\wedge^{d} t_{i-(d-1)(g-1)}:{ }^{\tau} \mathcal{F}_{i} \cong \wedge^{d \tau} \mathcal{E}_{i-(d-1)(g-1)} \rightarrow \mathcal{F}_{i+1}$, and $j_{i}^{\prime \prime}=\wedge^{d} j_{i-(d-1)(g-1)}:$ $\mathcal{F}_{i} \rightarrow \mathcal{F}_{i+1}$, respectively.

Lemma 4.3. The triple $\left(\mathcal{F}_{i}, t_{i}^{\prime \prime}, j_{i}^{\prime \prime}\right)_{i \in \mathbb{Z}}$ is an elliptic sheaf of rank one.

Proof. We need to check Conditions (1)-(5) in Section 4.2 .1 for $\left(\mathcal{F}_{i}, t_{i}^{\prime \prime}, j_{i}^{\prime \prime}\right)_{i \in \mathbb{Z}}$. Condition (1) follows from the functoriality of exterior power operations. Conditions (3) and (4) follow from Lemma 4.4 below. Condition (5) follows since the highest exterior power operation does not change the degree of locally free sheaves. Hence it remains to check Condition (2).

Let us give a proof that $\mathcal{F}_{i+\operatorname{deg}(\infty)} \cong \mathcal{F}_{i}(\infty)$. We may assume that $S$ is connected and is of finite type over $\operatorname{Spec} A$. By Conditions (2) and (3) in the definition of elliptic sheaves, the scheme $\{\infty\} \times S$ decomposes into a disjoint union $\{\infty\} \times$ $S=\coprod_{j=1}^{\operatorname{deg}(\infty)} S_{j}$ of $\operatorname{deg}(\infty)$ connected components, and for each $i$ the $\mathcal{O}_{C \times \times_{\text {Spec } \mathbb{F}_{q}} S^{-}}$ module $\mathcal{F}_{i+1} / j_{i}^{\prime \prime}\left(\mathcal{F}_{i}\right)$ is the direct image of an invertible module on $S_{j_{i}}$ for some $j_{i}$. By Conditions (3) and (4) in the definition of elliptic sheaves, the map $t_{i}$ 
induces an isomorphism ${ }^{\tau}\left(\mathcal{F}_{i} / \mathcal{F}_{i-1}\right) \cong \mathcal{F}_{i+1} / \mathcal{F}_{i}$ for each $i$. This implies that the components $S_{j_{i}}, \cdots, S_{j_{i+\operatorname{deg}(\infty)-1}}$ are pairwise distinct. Hence $\mathcal{F}_{i+\operatorname{deg}(\infty)} / \mathcal{F}_{i}$ is the direct image of an invertible module on $\{\infty\} \times S$. Since $\mathcal{F}_{i+\operatorname{deg}(\infty)} / \mathcal{F}_{i}$ is a submodule of $\mathcal{F}_{i+d \cdot \operatorname{deg}(\infty)} / \mathcal{F}_{i} \cong \mathcal{F}_{i}(d \infty) / \mathcal{F}_{i}$, it equals $\mathcal{F}_{i}(-\infty) / \mathcal{F}_{i}$

Lemma 4.4. If $f: \mathcal{E} \rightarrow \mathcal{E}^{\prime}$ is a homomorphism of locally free sheaves of rank $d$ on a scheme $T$ such that Coker $f$ is isomorphic to the direct image of an invertible sheaf on a closed subscheme $T^{\prime} \subset T$, then the cokernel of the homomorphism $\wedge^{d} \mathcal{E} \rightarrow \wedge^{d} \mathcal{E}^{\prime}$ is also isomorphic to the direct image of an invertible sheaf on $T^{\prime}$.

Proof. It suffices to prove the case where $T=\operatorname{Spec} R$ is the spectrum of a commutative local ring $R$, and $T^{\prime}$ is the closed point of $T$. Let $f: R^{\oplus d} \rightarrow R^{\oplus d}$ be an $R$-linear endomorphism which is expressed by a $d \times d$ matrix $F$. Suppose that the cokernel Coker $f$ of $f$ is generated as an $R$-module by at most one element. The claim is that Coker $f$ is isomorphic to $R /(\operatorname{det} F)$.

Let $e \in R^{\oplus d}$ be an element in the preimage of a generator of Coker $f$ under the quotient map $R^{\oplus d} \rightarrow R^{\oplus d} / f\left(R^{\oplus d}\right)=$ Coker $f$. Let $e_{1}, \ldots, e_{d}$ be the standard basis of $R^{\oplus d}$.

It follows from Nakayama's lemma that there exists an integer $i \in\{1, \ldots, d\}$ such that $R^{\oplus d}$ is generated by $e$ and $\left\{f\left(e_{j}\right) \mid j \neq i\right\}$. Let $h: R^{\oplus d} \rightarrow R^{\oplus d}$ be the endomorphism which sends $e_{i}$ to $e$ and sends $e_{j}$ to $f\left(e_{j}\right)$ for $j \neq i$. Then $h$ is surjective.

Let $\mathfrak{m} \subset R$ denote the maximal ideal. Because $h$ is an isomorphism modulo $\mathfrak{m}$, its determinant is non-zero modulo $\mathfrak{m}$ which means that $\operatorname{det}(h)$ is invertible. This implies, using Cramer's rule, that $h$ is an isomorphism.

Hence $\{e\} \cup\left\{f\left(e_{j}\right) \mid j \neq i\right\}$ forms a basis of $R^{\oplus d}$. Write $f\left(e_{i}\right)$ in the form $f\left(e_{i}\right)=b e+\sum_{j \neq i} b_{j} f\left(e_{j}\right)$ with $b, b_{j} \in R$. Then we see that Coker $f \cong R /(b)$. On the other hand the composite $h^{-1} \circ f$ sends $e_{i}$ to $b e_{i}+\sum_{j \neq i} b_{j} e_{j}$ and sends $e_{j}$ to $e_{j}$ for $j \neq i$. Hence the determinant of the $d \times d$ matrix corresponding to $h^{-1} \circ f$ is equal to $b$. This proves the claim.

4.4.2. Using the isomorphism which sends the element $(1,0, \ldots, 0) \wedge \cdots \wedge(0, \ldots, 0,1)$

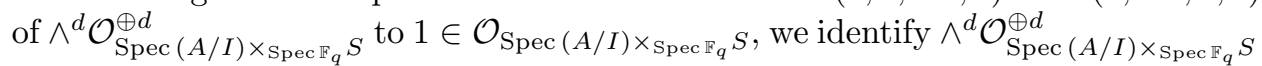
with $\mathcal{O}_{\text {Spec }}(A / I) \times_{\operatorname{Spec}_{q}} S$. Since $\mathcal{F}_{0}=\wedge^{d} \mathcal{E}_{0}$, the level $I$ structure

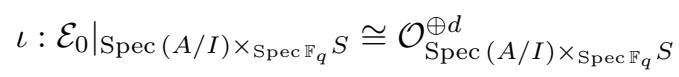

on $\left(\mathcal{E}_{i}, t_{i}, j_{i}\right)_{i \in \mathbb{Z}}$ induces a level $I$ structure

$$
\wedge^{d} \iota:\left.\mathcal{F}_{0}\right|_{\operatorname{Spec}(A / I) \times_{\operatorname{Spec} \mathbb{F}} S} \cong \mathcal{O}_{\operatorname{Spec}(A / I) \times_{\operatorname{Spec~} \mathbb{F}} S}
$$

on $\left(\mathcal{F}_{i}, t_{i}^{\prime \prime}, j_{i}^{\prime \prime}\right)_{i \in \mathbb{Z}}$.

The correspondence, which takes an elliptic sheaf to its determinant elliptic sheaf, gives rise to a morphism

$$
w_{C \backslash \operatorname{Spec}(A / I)}: M_{I, C \backslash \operatorname{Spec}(A / I)}^{d} \rightarrow M_{I, C \backslash \operatorname{Spec}(A / I)}^{1}
$$

over $C \backslash \operatorname{Spec}(A / I)$. We set $U_{I}=\operatorname{Spec} A \backslash \operatorname{Spec}(A / I)$. The morphism $w_{C \backslash \operatorname{Spec}(A / I)}$ induces the morphism $M_{I}^{d} \times_{\operatorname{Spec} A} U_{I} \rightarrow M_{I}^{1} \times_{\operatorname{Spec} A} U_{I}$. Suppose that $\operatorname{Spec}(A / I)$ contains two or more closed points. From Lemma 4.2 , we know that the scheme $M_{I}^{1}$ is isomorphic to the integral closure of $\operatorname{Spec} A$ in $M_{I}^{1} \times_{\operatorname{Spec} A} U_{I}$. Since $M_{I}^{d}$ is regular (in particular normal), the morphism $M_{I}^{d} \times_{\operatorname{Spec} A} U_{I} \rightarrow M_{I}^{1} \times_{\operatorname{Spec} A} U_{I}$ 
extends uniquely to a morphism $w: M_{I}^{d} \rightarrow M_{I}^{1}$ by [EGAII, (6.3.9), p. 119]. We call the morphism $w$ the determinant morphism.

4.5. Adelic description of the determinant morphism. Let $d$ be a positive integer. Since $\mathfrak{X}_{1}$ is a point, there exists a canonical morphism

$$
\begin{aligned}
r: & \mathrm{GL}_{d}(k) \backslash\left(\mathrm{GL}_{d}\left(\mathbb{A}^{\infty}\right) / \mathbb{K}_{d, I} \times \mathfrak{X}_{d}\right) \rightarrow \mathrm{GL}_{d}(k) \backslash\left(\mathrm{GL}_{d}\left(\mathbb{A}^{\infty}\right) / \mathbb{K}_{d, I} \times \mathfrak{X}_{1}\right) \\
& \stackrel{r^{\prime}}{\longrightarrow} k^{\times} \backslash\left(\left(\mathbb{A}^{\infty}\right)^{\times} / \mathbb{K}_{1, I} \times \mathfrak{X}_{1}\right)
\end{aligned}
$$

where the last map $r^{\prime}$ is the one induced by the determinant homomorphism det : $\mathrm{GL}_{d}\left(\mathbb{A}^{\infty}\right) \rightarrow\left(\mathbb{A}^{\infty}\right)^{\times}$.

Proposition 4.5. The morphism $r^{\prime}$ in (4.3) is bijective. The morphism $r$ in (4.3) is compatible with the determinant morphism $w: M_{I}^{d} \rightarrow M_{I}^{1}$, that is, the diagram

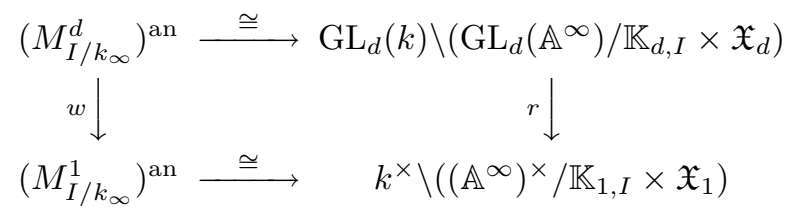

is commutative. Here, by abuse of notation, we let $w$ denote the analytification of the base change to $k_{\infty}$ of the determinant morphism $w$. The horizontal isomorphisms are those of (4.2).

Proof. The surjectivity of $r^{\prime}$ is clear. To show the injectivity, let $\mathfrak{X}_{1}=\{x\}$ and suppose that $r^{\prime}\left(\mathrm{GL}_{d}(k) g_{1} \mathbb{K}_{d, I}, x\right)=r^{\prime}\left(\mathrm{GL}_{d}(k) g_{2} \mathbb{K}_{d, I}, x\right)$. Replacing $g_{2}$ by another element in $\mathrm{GL}_{d}(k) g_{2} \mathbb{K}_{d, I}$, we may assume that $\operatorname{det}\left(g_{1}\right)=\operatorname{det}\left(g_{2}\right)$. Since $\mathrm{SL}_{d}(k)$ is dense in $\mathrm{SL}_{d}\left(\mathbb{A}^{\infty}\right)$ by the strong approximation theorem, the intersection $\mathrm{SL}_{d}(k) \cap$ $g_{1} \mathbb{K}_{d, I} g_{2}^{-1}$ is non-empty. This implies that $\mathrm{GL}_{d}(k) g_{1} \mathbb{K}_{d, I}=\mathrm{GL}_{d}(k) g_{2} \mathbb{K}_{d, I}$. Hence $r^{\prime}$ is injective.

Let $\widehat{w}_{\infty}: \widehat{M}_{I, \infty}^{d} \rightarrow \widehat{M}_{I, \infty}^{1}$ be the morphism of formal stacks over Spf $\mathcal{O}_{\infty}$ induced by the morphism $w_{C \backslash \operatorname{Spec}(A / I)}$. We will show that the following diagram

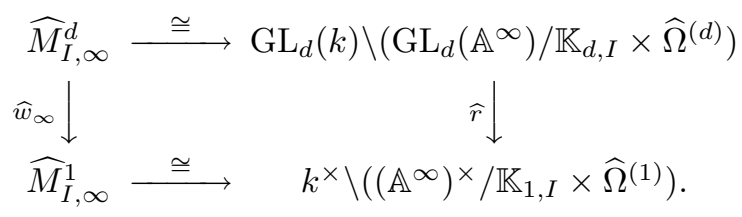

where the horizontal maps are the isomorphisms given in (4.1) and the right vertical map $\widehat{r}$ is the one induced by det $: \mathrm{GL}_{d}\left(\mathbb{A}^{\infty}\right) \rightarrow\left(\mathbb{A}^{\infty}\right)^{\times}$and the structure morphism $\widehat{\Omega}^{(d)} \rightarrow \operatorname{Spf} \mathcal{O}_{\infty}=\widehat{\Omega}^{(1)}$, is commutative. Then by taking the generic fiber in the sense of Raynaud, we obtain the commutativity of the diagram (4.4). Since the isomorphism (4.1) is obtained as the composite

$$
\widehat{M}_{I, \infty}^{d} \stackrel{\alpha_{d}}{\longrightarrow} \mathrm{GL}_{d}(k) \backslash\left(\mathrm{GL}_{d}\left(\mathbb{A}^{\infty}\right) / \mathbb{K}_{I} \times \widehat{\mathrm{Ell}}{ }^{(d)}\right) \cong \mathrm{GL}_{d}(k) \backslash\left(\mathrm{GL}_{d}\left(\mathbb{A}^{\infty}\right) / \mathbb{K}_{I} \times \widehat{\Omega}^{(d)}\right),
$$

where the second isomorphism is the one induced by the isomorphism $\widehat{\operatorname{Ell}}^{(d)} \cong$ $\widehat{\Omega}^{(d)}$ which we have explained in Section 4.3.4, the claim follows from Lemma 4.6 below. 
Lemma 4.6. The following diagram of formal stacks is commutative:

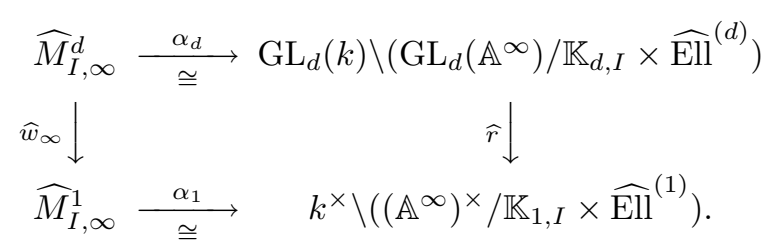

Here the right vertical map is the one induced by $\operatorname{det}: \mathrm{GL}_{d}\left(\mathbb{A}^{\infty}\right) \rightarrow\left(\mathbb{A}^{\infty}\right)^{\times}$.

Proof. Let $R$ be an object in Nilp. It suffices to prove that the diagram

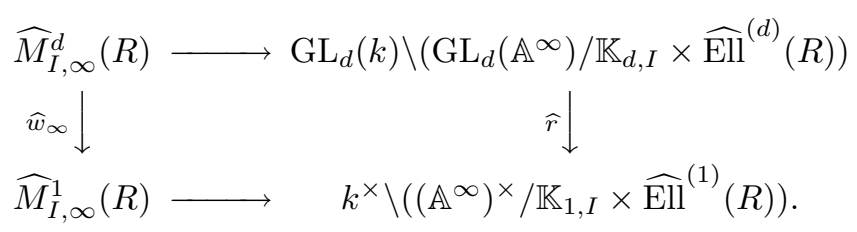

of groupoids is commutative up to functorial isomorphisms. Let $y$ be an object in $\widehat{M}_{I, \infty}^{d}(R)$, that is, $y$ is an elliptic sheaf $\mathcal{E}=\left(\mathcal{E}_{i}, j_{i}, t_{i}\right)$ over $\operatorname{Spec} R$ with a level $I$ structure. Let $z=\widehat{w}_{\infty}(y)$. Then, by definition, $z$ is an elliptic sheaf $\mathcal{F}=$ $\left(\mathcal{F}_{i}, j_{i}^{\prime \prime}, t_{i}^{\prime \prime}\right)$ over Spec $R$ with a level $I$ structure, where $\mathcal{F}_{i}=\wedge^{d} \mathcal{E}_{i-(d-1)(g-1)}, t_{i}^{\prime \prime}=$ $\wedge^{d} t_{i-(d-1)(g-1)}$ and $j_{i}^{\prime \prime}=\wedge^{d} j_{i-(d-1)(g-1)}$.

For a commutative ring $L$, let $\sigma_{L}: \wedge^{d}\left(L^{\oplus d}\right) \cong L$ denote the $L$-linear isomorphism which sends the element $(1,0, \ldots, 0) \wedge \cdots \wedge(0, \ldots, 0,1)$ of $\wedge^{d}\left(L^{\oplus d}\right)$ to $1 \in L$. Let $P_{\mathcal{E}}$, $Q_{\mathcal{E}}^{\prime}, Q_{\mathcal{E}}$, and $\mu_{\mathcal{E}}$ be as in Section 4.3.3. We can take $P_{\mathcal{F}}$ and $\mu_{\mathcal{F}}$ so that $P_{\mathcal{F}}=\wedge^{d} P_{\mathcal{E}}$ and $\mu_{\mathcal{F}}=\sigma_{k} \circ \wedge^{d} \mu_{\mathcal{E}}$. Let $T=$ Spec $R^{\prime}$ be a connected affine scheme over Spec $R$ such that the $\operatorname{Aut}_{A}\left(P_{\mathcal{E}}\right)$-set $Q_{\mathcal{E}}^{\prime}(T)$ is non-empty. Take an element $\psi_{\mathcal{E}}$ in $Q_{\mathcal{E}}^{\prime}(T)$. Then the isomorphism $\psi_{\mathcal{F}}:=\wedge^{d} \psi_{\mathcal{E}}$ is an element in $Q_{\mathcal{F}}^{\prime}(T)$.

Let $\Lambda_{\psi_{\mathcal{E}}, i}$ for each $i \in \mathbb{Z}$, and $\widetilde{\iota}_{\psi_{\mathcal{E}, \wp}}, g_{\psi_{\mathcal{E}, \wp}}$ for each prime $\wp \neq \infty$ of $k$ be as in Section 4.3.3. Then we can take $\widetilde{\iota}_{\psi_{\mathcal{F}, \wp}}$ so that $\widetilde{\iota}_{\psi_{\mathcal{F}, \wp}}=\wedge^{d} \widetilde{\iota}_{\psi_{\mathcal{E}}, \wp} \circ \sigma_{A_{\wp}}^{-1}$. Let $\Lambda_{\psi_{\mathcal{F}, i}}$ for each $i \in \mathbb{Z}$ be as in Section 4.3.3. Then we have $\Lambda_{\psi_{\mathcal{F}}, i}=\sigma_{k_{\infty} \widehat{\otimes}_{\mathbb{F}_{q}} R^{\prime}}\left(\wedge^{d} \Lambda_{\psi_{\mathcal{E}}, i}\right)$. By the definition of the matrix $g_{\psi_{\mathcal{E}}, \wp}$, the composite

$$
\begin{gathered}
\wedge^{d} k_{\wp}^{\oplus d}=\left(\wedge^{d} A_{\wp}^{\oplus d}\right) \otimes_{A_{\wp}} k_{\wp} \stackrel{\wedge^{d}{\widetilde{\tau_{\psi}}}_{\mathcal{E}, \wp} \otimes \mathrm{id}}{\longrightarrow}\left(\wedge^{d}\left(P \otimes_{A} A_{\wp}\right)\right) \otimes_{A_{\wp}} k_{\wp} \\
=\left(\wedge^{d}\left(P \otimes_{A} k\right)\right) \otimes_{k} k_{\wp} \stackrel{\wedge^{d} \mu_{\mathcal{E}} \otimes \mathrm{id}}{\longrightarrow}\left(\wedge^{d} k^{\oplus d}\right) \otimes_{k} k_{\wp}=\wedge^{d} k_{\wp}^{\oplus d}
\end{gathered}
$$

is supplied by the multiplication by $\operatorname{det} g_{\psi_{\mathcal{E}, \wp}}$. Hence we see from the description of the isomorphism $\alpha_{d}$ given in Section 4.3.3 that the image of $z$ under the equivalence $\widehat{M}_{I, \infty}^{1}(R) \stackrel{\cong}{\longrightarrow}\left(k^{\times} \backslash\left(\left(\mathbb{A}^{\infty}\right)^{\times} / \mathbb{K}_{1, I} \times \widehat{\mathrm{Ell}}^{(1)}\right)(R)\right.$ is equal to the $k^{\times}$equivariant morphism $k^{\times} \times \operatorname{Aut}_{A}\left(P_{\mathcal{F}}\right) Q_{\mathcal{F}}^{\prime} \rightarrow k^{\times} \backslash\left(\left(\mathbb{A}^{\infty}\right)^{\times} / \mathbb{K}_{1, I} \times\left(\widehat{\operatorname{Ell}}^{(1)} \times{ }_{\operatorname{Spf} \mathcal{O}_{\infty}}\right.\right.$ $\operatorname{Spec}(R))$ which is induced by the $\operatorname{Aut}\left(P_{\mathcal{F}}\right)$-equivariant morphism $\alpha_{1}^{\prime}(z): Q_{\mathcal{F}}^{\prime} \rightarrow$ $k^{\times} \backslash\left(\left(\mathbb{A}^{\infty}\right)^{\times} / \mathbb{K}_{1, I} \times\left(\widehat{\operatorname{Ell}}^{(1)} \times \operatorname{Spf} \mathcal{O}_{\infty} \operatorname{Spec}(R)\right)\right.$ characterized by the following property: for each connected affine scheme $T=\operatorname{Spec} R^{\prime}$ over $\operatorname{Spec} R$ and for each element $\psi_{\mathcal{E}}$ in $Q_{\mathcal{E}}^{\prime}(T)$, the image $\alpha_{1}^{\prime}(z)\left(\psi_{\mathcal{F}}\right)$ of $\psi_{\mathcal{F}} \in Q_{\mathcal{F}}^{\prime}(T)$ under $\alpha_{1}^{\prime}(z)$ is class of $\left(\left(\operatorname{det} g_{\psi_{\mathcal{E}, \wp}}\right)_{\wp \neq \infty},\left(\Lambda_{\psi_{\mathcal{F}}, i}, \sigma_{k_{\infty} \widehat{\otimes}_{\mathbb{F}_{q}} R^{\prime}} \circ \wedge^{d} t_{i}\right)_{i \in \mathbb{Z}}\right) \in\left(\mathbb{A}^{\infty}\right)^{\times} \times \widehat{\operatorname{Ell}}^{(1)}\left(R^{\prime}\right)$. This proves the claim. 


\section{DEURING'S THEORY FOR DRINFELD MODULES OF RANK TWO}

The main goal of this section is to construct an isomorphism (5.4), and to prove Proposition 5.4. They are the only results to be used in other sections.

Deuring's theory for elliptic curves applies to the Drinfeld modular context. (We refer to the introduction in [Gek3] for the historical account including the case of Drinfeld modules.) This gives a description of the category of supersingular Drinfeld modules of rank two in terms of the category of left $\mathcal{O}$-modules, where $\mathcal{O}$ is the endomorphism ring of a supersingular Drinfeld module.

As a consequence of the equivalence of categories (Proposition 5.1), we obtain the isomorphism (5.4), describing the set of supersingular points as a double coset space. This gives rise to an action of the non- $(\wp, \infty)$-part $\operatorname{GL}_{2}\left(\mathbb{A}^{\wp, \infty}\right)$ of the adeles. On the other hand, there is a natural action of $\mathrm{GL}_{2}\left(\mathbb{A}^{\infty}\right)$ coming from the action on the moduli spaces as described by Drinfeld. We show in Proposition 5.4 that the two actions on the set of supersingular points are compatible.

The story is classical but we were not able to find a written account of this material. We think it is necessary to give the details when one is to check the compatibility of the actions (Proposition 5.4).

In Section 5.1, we recall the definition of Drinfeld modules for completeness. Section 5.2.1 is devoted to Deuring's theory (Proposition 5.1). We note that the correspondence between the isomorphism classes (i.e., the objects of the categories) are usually found in the literature; we need the correspondence between the morphisms and of some isogenies. In Section 5.2.2, we consider the case with level structures. Section 5.2.3 contains the main statements (the isomorphism (5.4) and Proposition 5.4) to be used in Lemmas 7.1 and 7.2.

\subsection{Drinfeld modules.}

5.1.1. We recall the definition of a Drinfeld module. Let $S$ be a scheme over Spec $A$. Let $d$ be a positive integer. A Drinfeld module of rank $d$ over $S$ is a scheme $E$ of $A$-modules over $S$ satisfying the following three conditions.

(1) Zariski locally on $S$, the scheme $E$ is isomorphic to additive group scheme $\mathbb{G}_{a}$ as a commutative group scheme.

(2) If we denote the $A$-action on $E$ by $\varphi: A \rightarrow \operatorname{End}_{S \text {-group }}(E)$, then, for every $a \in A \backslash\{0\}$, the $a$-action $\varphi(a): E \rightarrow E$ on $E$ is finite, locally free of constant degree $|a|_{\infty}^{d}$.

(3) The $A$-action on Lie $E$ induced by $\varphi$ coincides with the $A$-action on Lie $E$ which comes from the structure homomorphism $A \rightarrow \Gamma\left(S, \mathcal{O}_{S}\right)$.

5.1.2. Drinfeld level structure and modular varieties. Let $d$ be a positive integer. Let $E$ be a Drinfeld module of rank $d$ over a scheme $S$ over $\operatorname{Spec} A$. Let $E(S)$ be the $A$-module of the sections of $E \rightarrow S$. We regard an element in $E(S)$ as an effective Cartier divisor in $E / S$ (in the sense of [Ka-Ma, 1.1.1, p. 3]). Let $I \subset A$ be a non-zero ideal. Let $E[I]$ denote the $I$-torsion part of $E$. If we take generators $a_{1}, \ldots, a_{m} \in I$ of $I$, then $E[I]$ is equal to the fiber product of the zero section $S \rightarrow E^{m}$ (here $E^{m}$ denotes the $m$-fold fiber product of $E$ over $S$ ) and the morphism $E \rightarrow E^{m}$ which is the composition of the morphism $\left(a_{1}, \ldots, a_{m}\right): E^{m} \rightarrow E^{m}$ with the diagonal embedding $E \rightarrow E^{m}$. A Drinfeld level $I$ structure on $E$ is a homomorphism $\phi:\left(I^{-1} / A\right)^{\oplus d} \rightarrow E(S)$ of $A$-modules such that $\sum_{a \in\left(I^{-1} / A\right)^{\oplus d}} \phi(a)$ equals $E[I]$ as an effective Cartier divisor in $E / S$. 
5.2. Supersingular Drinfeld modules of rank two. A Drinfeld module of rank two over Spec $\overline{\kappa(\wp)}$ is called ordinary (resp. supersingular) if the corresponding formal $A_{\wp}$-module is of height one (resp. two) (see [Dr1, §1, p. 563] for the definition of formal $A_{\wp}$-modules). We refer to [Gek3, Proposition 4.1, p. 195] for other equivalent definitions.

5.2.1. Adelic description. A supersingular Drinfeld module $E$ of rank two over $\operatorname{Spec} \overline{\kappa(\wp)}$ is known to exist. We fix one such $E$ and denote it by $E_{0}$. Put

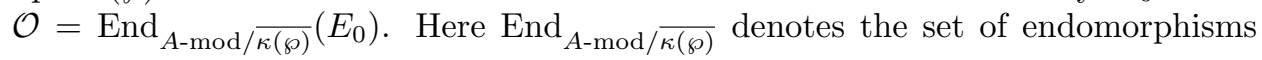
of schemes of $A$-modules over $\operatorname{Spec} \overline{\kappa(\wp)}$. Then $\mathcal{O}$ is an $A$-algebra. Moreover, $B=\mathcal{O} \otimes_{A} k$ is a quaternion algebra over $k$ which ramifies exactly at $\wp$ and at $\infty$, and $\mathcal{O}$ is a maximal $A$-order of $B$ (this is due to Drinfeld ([Dr2, §2, p. 161-163])). We say that a left $\mathcal{O}$-module $M$ is invertible if $M \otimes_{A} A_{\wp^{\prime}}$ is a free $\mathcal{O} \otimes_{A} A_{\wp^{\prime}}$-module of rank one for each prime $\wp^{\prime} \neq \infty$. Let $M_{1}$ and $M_{2}$ be two invertible left $\mathcal{O}$ modules. We say that a homomorphism $M_{1} \rightarrow M_{2}$ of left $\mathcal{O}$-modules is an isogeny if it is injective. We say that an isogeny $M_{1} \rightarrow M_{2}$ is prime to $\wp$ if its cokernel has no $\wp$-torsion.

For a supersingular Drinfeld module $E$ of rank two over $\operatorname{Spec} \overline{\kappa(\wp)}$, we put $\mathcal{F}_{E_{0}}(E)=\operatorname{Hom}_{A-\bmod / \overline{\kappa(\wp)}}\left(E, E_{0}\right)$. Here $\operatorname{Hom}_{A-\bmod / \overline{\kappa(\wp)}}$ denotes the set of homomorphisms of schemes of $A$-modules over $\operatorname{Spec} \overline{\kappa(\wp)}$. The composition law

$$
\operatorname{End}_{A-\bmod / \overline{\kappa(\wp)}}\left(E_{0}\right) \times \operatorname{Hom}_{A-\bmod / \overline{\kappa(\wp)}}\left(E, E_{0}\right) \rightarrow \operatorname{Hom}_{A-\bmod / \overline{\kappa(\wp)}}\left(E, E_{0}\right)
$$

provides $\mathcal{F}_{E_{0}}(E)$ with a structure of a left $\mathcal{O}$-module. Let $E^{\prime}$ be another supersingular Drinfeld module of rank two over Spec $\overline{\kappa(\wp)}$ and let $f: E \rightarrow E^{\prime}$ be a morphism of schemes of $A$-modules over Spec $\overline{\kappa(\wp)}$. Then the map $\mathcal{F}_{E_{0}}(f): \mathcal{F}_{E_{0}}\left(E^{\prime}\right) \rightarrow \mathcal{F}_{E_{0}}(E)$ given by the composition with $f$ is a homomorphism of left $\mathcal{O}$-modules.

For a scheme $G$ of $A$-modules over Spec $\overline{\kappa(\wp)}$, we denote by $G(\overline{\kappa(\wp)})$ the $A$ module of scheme theoretic sections of $G \rightarrow \operatorname{Spec} \overline{\kappa(\wp)}$. We let $M_{0}$ denote the torsion $A$-module $E_{0}(\overline{\kappa(\wp)})$ equipped with a canonical left $\mathcal{O}$-module structure.

Proposition 5.1. Let us consider the following two categories.

(1) The category whose objects are the supersingular Drinfeld modules of rank two over Spec $\overline{\kappa(\wp)}$, and whose morphisms are the morphisms of schemes of A-modules over $\operatorname{Spec} \overline{\kappa(\wp)}$.

(2) The category whose objects are the invertible left $\mathcal{O}$-modules, and whose morphisms are the homomorphisms of left $\mathcal{O}$-modules.

Then the contravariant functor $\mathcal{F}=\mathcal{F}_{E_{0}}$ gives an anti-equivalence from the category (1) to the category (2).

Moreover the notions of isogeny and prime-to- $\wp$ isogeny in both categories coincide. If $E_{1} \rightarrow E_{2}$ is a prime-to-ø isogeny in the category (1), then there is a canonical isomorphism

$$
\operatorname{Coker}\left[\mathcal{F}\left(E_{2}\right) \rightarrow \mathcal{F}\left(E_{1}\right)\right] \cong \operatorname{Hom}_{A}\left(\operatorname{Ker}\left[E_{1}(\overline{\kappa(\wp)}) \rightarrow E_{2}(\overline{\kappa(\wp)})\right], M_{0}\right) .
$$

of left $\mathcal{O}$-modules. Here Coker (resp. Ker) is the cokernel in the category of left $\mathcal{O}$-modules (resp. the kernel in the category of A-modules) and the structure of a left $\mathcal{O}$-module on $\operatorname{Hom}_{A}\left(\operatorname{Ker}\left[E_{1}(\overline{\kappa(\wp)}) \rightarrow E_{2}(\overline{\kappa(\wp)})\right], M_{0}\right)$ is the one induced by the left action of $\mathcal{O}$ on $M_{0}$. 
Remark 5.2. The essential part of this proposition is a consequence of the results in $\S 2$ of [Dr2, p. 161-163]. Let us remark that the description using left ideals is an analogue of Deuring's result for elliptic curves and is adapted from [Gek3].

Proof. First we show that for any supersingular Drinfeld module $E$ of rank 2 over $\operatorname{Spec} \overline{\kappa(\wp)}$, the left $\mathcal{O}$-module $\mathcal{F}(E)=\operatorname{Hom}_{A \text {-mod } / \overline{\kappa(\wp)}}\left(E, E_{0}\right)$ is an invertible left $\mathcal{O}$-module. Note that any two Drinfeld modules of rank 2 over $\operatorname{Spec} \overline{\kappa(\wp)}$ are isomorphic to each other. Note that any two supersingular Drinfeld modules over Spec $\overline{\kappa(\wp)}$ are isogenous. Hence $\mathcal{F}(E)$ is not zero. Since any isogeny $E_{0} \rightarrow E$ induces an injective homomorphism $\mathcal{F}(E) \hookrightarrow \mathcal{F}\left(E_{0}\right)=\mathcal{O}$ of left $\mathcal{O}$-modules, the left $\mathcal{O}$-module $\mathcal{F}(E)$ is isomorphic to a non-zero left ideal of $\mathcal{O}$. Since $\mathcal{O}$ is a maximal order of the quaternion division algebra $B$, any non-zero left ideal of $\mathcal{O}$ is an invertible left $\mathcal{O}$-module. Therefore $\mathcal{F}(E)$ is an invertible left $\mathcal{O}$-module.

Let $\mathcal{C}$ denote the full subcategory of the category (2) whose objects are the non-zero left ideals of $\mathcal{O}$. We note that the canonical inclusion functor from $\mathcal{C}$ to the category (2) gives an equivalence of categories. We construct a contravariant functor $\mathcal{G}$ from $\mathcal{C}$ to the category (1) which gives a quasi-inverse to the functor $\mathcal{F}$.

In [Gek3, 3.7. Lemma, p. 193], Gekeler constructed, for any non-zero left $\mathcal{O}$ ideal $\mathfrak{A} \subset \mathcal{O}$, a supersingular Drinfeld module $\mathcal{G}(\mathfrak{A})$ over $\operatorname{Spec} \overline{\kappa(\wp)}$. Let us recall the construction of $\mathcal{G}(\mathfrak{A})$. Let $\tau$ denote the endomorphism of the group scheme $\mathbb{G}_{a}$ over Spec $\overline{\kappa(\wp)}$ which induces, for any scheme $S$ over Spec $\overline{\kappa(\wp)}$, the $q$-power endomorphism of the ring $\mathbb{G}_{a}(S)=\Gamma\left(S, \mathcal{O}_{S}\right)$, where $q$ is as in Section 4.1. For $x \in \overline{\kappa(\wp)}$, we also denote by $x$ the endomorphism of $\mathbb{G}_{a}$ over Spec $\overline{\kappa(\wp)}$ which induces, for any scheme $S$ over Spec $\overline{\kappa(\wp)}$, the endomorphism of the additive group $\mathbb{G}_{a}(S)=\Gamma\left(S, \mathcal{O}_{S}\right)$ supplied by the multiplication by $x$. We fix an isomorphism $E_{0} \cong \mathbb{G}_{a}$ as group schemes over Spec $\overline{\kappa(\wp)}$. By abuse of notation, we denote by $\tau, x$ the endomorphisms of $E_{0}$ as a group scheme over Spec $\overline{\kappa(\wp)}$ corresponding to the endomorphisms $\tau, x$, respectively. Let $R$ be the subring of the ring of endomorphisms of $E_{0}$ as a group scheme over $\operatorname{Spec} \overline{\kappa(\wp)}$, which is generated by $\tau$ and by all $x \in \overline{\kappa(\wp)}$. Then $R$ is a non-commutative polynomial algebra over $\overline{\kappa(\wp)}$ in one variable $\tau$. The ring $R$ contains no zero divisor and any left ideal of $R$ is generated by one element. Note that the $\operatorname{ring} \mathcal{O}=\operatorname{End}_{A-\bmod / \overline{\kappa(\wp)}}\left(E_{0}\right)$ is a subring of $R$. For a left ideal $\mathfrak{A} \subset \mathcal{O}$, let $u_{\mathfrak{A}} \in R$ denote the unique monic polynomial in $\tau$ satisfying $R \mathfrak{A}=R u_{\mathfrak{A}}$. The structure of a scheme of $A$-modules over $\operatorname{Spec} \overline{\kappa(\wp)}$ on $E_{0}$ gives a ring homomorphism $\iota_{0}: A \rightarrow R$, and $\mathcal{O}$ equals the commutant of $\iota_{0}(A)$ in $R$. Let $a \in A$. Since $R u_{\mathfrak{A}} \iota_{0}(a)=R \mathfrak{A} \iota_{0}(a) \subset R \mathfrak{A}=R u_{\mathfrak{A}}$, there exists a unique element $\iota_{\mathfrak{A}}(a) \in R$ such that $u_{\mathfrak{A}} \iota_{0}(a)=\iota_{\mathfrak{A}}(a) u_{\mathfrak{A}}$. The map $\iota_{\mathfrak{A}}: A \rightarrow R$ is a ring homomorphism. The pair $\left(E_{0}, \iota_{\mathfrak{A}}\right)$ defines a Drinfeld module $\mathcal{G}(\mathfrak{A})$ of rank 2 over $\operatorname{Spec} \overline{\kappa(\wp)}$.

Let $\mathfrak{A}, \mathfrak{A}^{\prime} \subset \mathcal{O}$ be two left ideals, and let $f: \mathfrak{A} \rightarrow \mathfrak{A}^{\prime}$ be a homomorphism of left $\mathcal{O}$-modules. We construct a morphism $\mathcal{G}(f): \mathcal{G}\left(\mathfrak{A}^{\prime}\right) \rightarrow \mathcal{G}(\mathfrak{A})$ of Drinfeld modules over $\operatorname{Spec} \overline{\kappa(\wp)}$. There exists a unique element $b \in B$ such that the diagram

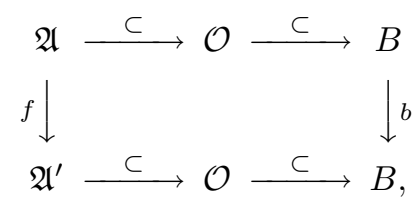


where the horizontal maps are the canonical inclusions and the right vertical map is supplied by the right multiplication by $b$, is commutative. Since the endomorphism $R \otimes_{\iota_{0}, A} k \rightarrow R \otimes_{\iota_{0}, A} k$ supplied by the right multiplication by $b$ sends an element in $R \mathfrak{A}$ to an element in $R \mathfrak{A}^{\prime}$, it induces a homomorphism $f_{R}: R \mathfrak{A} \rightarrow R \mathfrak{A}^{\prime}$ of left $R$-modules. By construction, the homomorphism $f_{R}$ commutes with the right multiplication by any element in $\iota_{0}(A)$. Recall that $R \mathfrak{A}=R u_{\mathfrak{A}}$ and $R \mathfrak{A}^{\prime}=R u_{\mathfrak{A}^{\prime}}$. Let $r_{f} \in R$ be the unique element satisfying $f_{R}\left(u_{\mathfrak{A}}\right)=r_{f} u_{\mathfrak{A}^{\prime}}$. Then for any $a \in A$ we have

$$
\begin{gathered}
r_{f} \iota_{\mathfrak{A}^{\prime}}(a) u_{\mathfrak{A}^{\prime}}=r_{f} u_{\mathfrak{A}^{\prime}} \iota_{0}(a)=f_{R}\left(u_{\mathfrak{A}}\right) \iota_{0}(a)=f_{R}\left(u_{\mathfrak{A}} \iota_{0}(a)\right) \\
=f_{R}\left(\iota_{\mathfrak{A}}(a) u_{\mathfrak{A}}\right)=\iota_{\mathfrak{A}}(a) f_{R}(u)=\iota_{\mathfrak{A}}(a) r_{f} u_{\mathfrak{A}^{\prime}} .
\end{gathered}
$$

Hence we have $r_{f} \iota_{\mathfrak{A}^{\prime}}(a)=\iota_{\mathfrak{A}}(a) r_{f}$ for any $a \in A$. Therefore, the element $r_{f} \in R$ gives a homomorphism $\mathcal{G}(f): \mathcal{G}\left(\mathfrak{A}^{\prime}\right) \rightarrow \mathcal{G}(\mathfrak{A})$ of Drinfeld modules over Spec $\overline{\kappa(\wp)}$. If $\mathfrak{A}^{\prime \prime} \subset \mathcal{O}$ is another left ideal and if $f^{\prime}: \mathfrak{A}^{\prime} \rightarrow \mathfrak{A}^{\prime \prime}$ is a homomorphism of left $\mathcal{O}$-modules, then it is easily checked that $\mathcal{G}\left(f^{\prime} \circ f\right)=\mathcal{G}(f) \circ \mathcal{G}\left(f^{\prime}\right)$. Hence $\mathcal{G}$ is a contravariant functor from the category $\mathcal{C}$ to the category (1). By [Gek3, 4.3. Theorem, p. 196], the functor $\mathcal{G}$ is essentially surjective.

We show that the composition $\mathcal{F} \circ \mathcal{G}$ is naturally isomorphic to the canonical inclusion functor from $\mathcal{C}$ to the category (2). Let $\mathfrak{A} \subset \mathcal{O}$ be a left ideal. The image of the injective homomorphism $\mathcal{F} \circ \mathcal{G}(\mathfrak{A}) \hookrightarrow \mathcal{F} \circ \mathcal{G}(\mathcal{O})=\mathcal{F}\left(E_{0}\right)=\mathcal{O}$ induced by the inclusion $\mathfrak{A} \subset \mathcal{O}$ is equal to the set $R \mathfrak{A} \cap \mathcal{O}$ which we denote by $\mathfrak{A}^{\prime}$. The set $\mathfrak{A}^{\prime}$ is a left ideal of $\mathcal{O}$ with $\mathfrak{A} \subset \mathfrak{A}^{\prime}$ and, since $R \mathfrak{A}=R \mathfrak{A}^{\prime}$, the homomorphism $\mathcal{G}\left(\mathfrak{A}^{\prime}\right) \rightarrow \mathcal{G}(\mathfrak{A})$ induced by the canonical inclusion $\mathfrak{A} \subset \mathfrak{A}^{\prime}$ is an isomorphism. Let nrd $: B \rightarrow k$ denote the reduced norm. By [Gek3, 3.10. Lemma, p. 194], the ideal of $A$ generated by $\left\{\operatorname{nrd}\left(b^{\prime}\right) \mid b^{\prime} \in \mathfrak{A}\right\}$ is equal to the ideal of $A$ generated by $\left\{\operatorname{nrd}\left(b^{\prime}\right) \mid b^{\prime} \in \mathfrak{A}^{\prime}\right\}$. Using this, one can check that $\mathfrak{A} \otimes_{A} A_{\wp^{\prime}}=\mathfrak{A}^{\prime} \otimes_{A} A_{\wp^{\prime}}$ as left ideals of $\mathcal{O} \otimes_{A} A_{\wp^{\prime}}$ for every nonzero prime ideal $\wp^{\prime} \subset A$. Hence we have $\mathfrak{A}=\mathfrak{A}^{\prime}$. We easily see that the isomorphism

$$
\mathcal{F} \circ \mathcal{G}(\mathfrak{A}) \cong \operatorname{Image}\left[\mathcal{F} \circ \mathcal{G}(\mathfrak{A}) \hookrightarrow \mathcal{F} \circ \mathcal{G}(\mathcal{O})=\mathcal{F}\left(E_{0}\right)=\mathcal{O}\right]=\mathfrak{A}
$$

gives a functorial isomorphism from $\mathcal{F} \circ \mathcal{G}$ to the canonical inclusion functor from $\mathcal{C}$ to the category $(2)$.

Therefore the functor $\mathcal{F}$ is essentially surjective and for any two supersingular Drinfeld modules $E, E^{\prime}$ of rank two over Spec $\overline{\kappa(\wp)}$, the map

$$
\operatorname{Hom}_{A-\bmod / \overline{\kappa(\wp)}}\left(E, E^{\prime}\right) \rightarrow \operatorname{Hom}_{\mathcal{O}}\left(\mathcal{F}\left(E^{\prime}\right), \mathcal{F}(E)\right)
$$

is surjective. Since both the source and the target of (5.1) are locally free $A$ modules of rank 4, the map (5.1) is an isomorphism. Hence $\mathcal{F}$ gives an equivalence of categories.

Let $\varphi: E_{1} \rightarrow E_{2}$ be a prime-to- $\wp$ isogeny of supersingular Drinfeld modules of rank 2 over $\operatorname{Spec} \overline{\kappa(\wp)}$. Let $X$ denote the kernel of $\varphi$. The exact sequence

$$
0 \rightarrow X \rightarrow E_{1} \stackrel{\varphi}{\longrightarrow} E_{2} \rightarrow 0
$$

of schemes of $A$-modules over Spec $\overline{\kappa(\wp)}$ gives an exact sequence

$$
0 \rightarrow \mathcal{F}\left(E_{2}\right) \rightarrow \mathcal{F}\left(E_{1}\right) \rightarrow \operatorname{Hom}_{A-\bmod / \overline{\kappa(\wp)}}\left(X, E_{0}\right)
$$

of left $\mathcal{O}$-modules. Since $\varphi$ is a prime-to- $\wp$ isogeny, we have an isomorphism $\operatorname{Hom}_{A-\bmod / \overline{\kappa(\wp)}}\left(X, E_{0}\right) \cong \bigoplus_{\wp^{\prime}} \operatorname{Hom}_{A-\bmod / \overline{\kappa(\wp)}}\left(X, E_{0}\right) \otimes_{A} A_{\wp^{\prime}}$, where $\wp^{\prime}$ runs over the primes of $k$ different from $\infty, \wp$. Let $\wp^{\prime}$ be a prime of $k$ different from $\infty, \wp$. For 
a scheme $G$ of $A$-modules over Spec $\overline{\kappa(\wp)}$, let $G\left[\wp^{\prime \infty}\right]$ denote the $A$-module of $\wp^{\prime}$ primary torsion elements of $G(\overline{\kappa(\wp)})$. Take a finite subfield $L \subset \overline{\kappa(\wp)}$ such that $E_{0}$, $E_{1}, E_{2}$, and $\varphi$ are defined over $L$. Then for $i=0,1,2$, the group $\operatorname{Gal}(\overline{\kappa(\wp)} / L)$ acts on the $A$-module $E_{i}\left[\wp^{\prime \infty}\right]$. By [Yu, Theorem 2, p. 164], we have an isomorphism

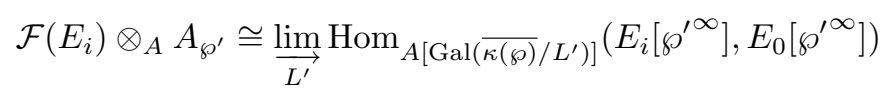

of left $\mathcal{O}$-modules for $i=1,2$, where $L^{\prime}$ runs over the finite subfields of $\overline{\kappa(\wp)}$ containing L. By [Yu, EXAMPLE 0, p. 166-167], the right hand side of (5.2) is isomorphic to $\operatorname{Hom}_{A}\left(E_{i}\left[\wp^{\prime \infty}\right], E_{0}\left[\wp^{\prime \infty}\right]\right)=\operatorname{Hom}_{A}\left(E_{i}\left[\wp^{\prime \infty}\right], M_{0}\right)$. The $A$-module $M_{0}$ is an injective $A$-module, since it is divisible as an $A$-module. Hence $\operatorname{Coker}\left[\mathcal{F}\left(E_{2}\right) \rightarrow\right.$ $\left.\mathcal{F}\left(E_{1}\right)\right] \otimes_{A} A_{\wp^{\prime}}$ is canonically isomorphic to

$$
\operatorname{Hom}_{A}\left(X\left[\wp^{\prime \infty}\right], E_{0}\left[\wp^{\prime \infty}\right]\right)=\operatorname{Hom}_{A}\left(X\left[\wp^{\prime \infty}\right], M_{0}\right) .
$$

Hence $\operatorname{Coker}\left[\mathcal{F}\left(E_{2}\right) \rightarrow \mathcal{F}\left(E_{1}\right)\right]$ is canonically isomorphic to $\operatorname{Hom}_{A}\left(X(\overline{\kappa(\wp)}), M_{0}\right)$.

5.2.2. For a ring $L$, let $\operatorname{Mat}_{2}(L)$ denote the $L$-algebra of two-by-two matrices with entries in $L$. Let $(k / A)^{\prime}=\bigoplus_{\wp^{\prime} \neq \wp, \infty} k_{\wp} / A_{\wp}$ be the prime-to- $\wp$ part of the torsion $A$ module $k / A$. Then $M_{0}$ is, as an $A$-module, isomorphic to the $\operatorname{direct} \operatorname{sum}(k / A)^{\prime \oplus 2}$ of two copies of $(k / A)^{\prime}$. We fix one such isomorphism $\xi:(k / A)^{\prime \oplus 2} \cong M_{0}$. Let $\widehat{A}^{\prime}=\prod_{\wp^{\prime} \neq \wp, \infty} A_{\wp^{\prime}}$ be the prime-to- $\wp$ part of $\widehat{A}$ and put $\widehat{\mathcal{O}}^{\prime}=\mathcal{O} \otimes_{A} \widehat{A}^{\prime}$. We regard $(k / A)^{\prime \oplus 2}$ as the $A$-module of column vectors. Then $(k / A)^{\prime \oplus 2}$ has a canonical structure of a left $\operatorname{Mat}_{2}\left(\widehat{A}^{\prime}\right)$-module and the action of $\operatorname{Mat}_{2}\left(\widehat{A}^{\prime}\right)$ on $(k / A)^{\prime \oplus 2}$ gives an isomorphism $\operatorname{Mat}_{2}\left(\widehat{A}^{\prime}\right) \cong \operatorname{End}_{A}\left((k / A)^{\prime \oplus 2}\right)$ of rings. The composite

$$
\mathcal{O} \rightarrow \operatorname{End}_{A}\left(M_{0}\right) \cong \operatorname{End}_{A}\left((k / A)^{\prime \oplus 2}\right) \cong \operatorname{Mat}_{2}\left(\widehat{A}^{\prime}\right)
$$

where the isomorphism in the middle is the one induced by the fixed isomorphism $\xi$, induces an isomorphism $\eta: \widehat{\mathcal{O}}^{\prime} \cong \operatorname{Mat}_{2}\left(\widehat{A}^{\prime}\right)$.

Let $E$ be a supersingular Drinfeld module of rank two over $\operatorname{Spec} \overline{\kappa(\wp)}$. Let $I \subset A$ be a non-zero ideal which is prime to $\wp$. To each level $I$ structure $\varphi:\left(I^{-1} / A\right)^{\oplus 2} \cong$ $E[I]$ on $E$, we associate the map

$$
\Phi_{E_{0}, \xi}(\varphi): \mathcal{F}_{E_{0}}(E) \rightarrow \mathcal{O} / I \mathcal{O}
$$

which is defined as the composite of the following four maps

- the canonical map $\mathcal{F}_{E_{0}}(E)=\operatorname{Hom}_{A \text {-mod } / \overline{\kappa(\wp)}}\left(E, E_{0}\right) \rightarrow \operatorname{Hom}_{A}\left(E(\overline{\kappa(\wp)}), M_{0}\right)$.

- the restriction map $\operatorname{Hom}_{A}\left(E(\overline{\kappa(\wp)}), M_{0}\right) \rightarrow \operatorname{Hom}_{A}\left(E[I](\overline{\kappa(\wp)}), M_{0}\right)$.

- the isomorphism

$$
\operatorname{Hom}_{A}\left(E[I](\overline{\kappa(\wp)}), M_{0}\right) \cong \operatorname{Hom}_{A}\left(\left(I^{-1} / A\right)^{\oplus 2}, M_{0}\right)
$$

induced by $\varphi$.

- the inverse of the isomorphism

$$
\mathcal{O} / I \mathcal{O} \cong \operatorname{Hom}_{A}\left(\left(I^{-1} / A\right)^{\oplus 2}, M_{0}\right)
$$

of left $\mathcal{O}$-modules which sends $1 \in \mathcal{O} / I \mathcal{O}$ to the restriction of the fixed isomorphism $\xi$ to the submodule $\left(I^{-1} / A\right)^{\oplus 2} \subset(k / A)^{\prime \oplus 2}$.

Then, as a consequence of the anti-equivalence in Proposition 5.1, we have the following. 
Corollary 5.3. Let $E$ be a supersingular Drinfeld module of rank two over $\operatorname{Spec} \overline{\kappa(\wp)}$. Let $I \subset A$ be a non-zero ideal which is prime to $\wp$.

(1) For each level I structure $\varphi:\left(I^{-1} / A\right)^{\oplus 2} \cong E[I]$ on $E$, the map $\Phi_{E_{0}, \xi}(\varphi)$ is a surjective homomorphism of left $\mathcal{O}$-modules.

(2) The map $\varphi \mapsto \Phi_{E_{0}, \xi}(\varphi)$ gives a bijection between the set of Drinfeld level I structures on $E$ and the set of surjective homomorphisms $\mathcal{F}_{E_{0}}(E) \rightarrow \mathcal{O} / I \mathcal{O}$ of left $\mathcal{O}$-modules.

5.2.3. We set $\widehat{\mathcal{O}}=\mathcal{O} \otimes_{A} \widehat{A}$. Let $\mathbb{K}_{I}^{\prime}$ denote the kernel of the homomorphism $(\widehat{\mathcal{O}})^{\times} \rightarrow(\mathcal{O} / I \mathcal{O})^{\times}$. For $x \in\left(B \otimes_{k} \mathbb{A}^{\infty}\right)^{\times}$, let $M_{x}$ denote the intersection $B \cap\left(\widehat{\mathcal{O}} \cdot x^{-1}\right)$ in $B \otimes_{k} \mathbb{A}^{\infty}$. Then $M_{x}$ is an invertible left $\mathcal{O}$-module and the composite

$$
M_{x} \hookrightarrow \widehat{\mathcal{O}} \cdot x^{-1} \cong \widehat{\mathcal{O}} \rightarrow \mathcal{O} / I \mathcal{O},
$$

where the injection on the left is the canonical inclusion and the isomorphism in the middle is the homomorphism supplied by the right multiplication by $x$, gives a surjection $M_{x} \rightarrow \mathcal{O} / I \mathcal{O}$.

Fix a supersingular Drinfeld module $E_{0}$ over Spec $\overline{\kappa(\wp)}$. Let $M_{0}$ be as in Proposition 5.1 and fix an isomorphism $\xi:(k / A)^{\oplus \oplus 2} \cong M_{0}$ of $A$-modules. By Proposition 5.1 and Corollary 5.3, the invertible left $\mathcal{O}$-module $M_{x}$ with the surjection $M_{x} \rightarrow \mathcal{O} / I \mathcal{O}$ defined above corresponds to a supersingular Drinfeld module $E_{x}$ of rank two over Spec $\overline{\kappa(\wp)}$ with a Drinfeld level $I$ structure $\varphi_{x}:\left(I^{-1} / A\right)^{\oplus 2} \cong E_{x}[I]$ on $E_{x}$.

By Proposition 5.1 and Corollary 5.3, the map which sends $x \in\left(B \otimes_{k} \mathbb{A}\right)^{\times}$to the supersingular Drinfeld module $E_{x}$ of rank two over Spec $\overline{\kappa(\wp)}$ with a Drinfeld level $I$ structure $\varphi_{x}$ gives a bijection (depending on $E_{0}$ and $\xi$ ) between the set $\Sigma_{I, \wp}$ of the isomorphism classes of supersingular Drinfeld modules of rank two over Spec $\overline{\kappa(\wp)}$ with a Drinfeld level $I$ structure and the double coset

$$
B^{\times} \backslash\left(B \otimes_{k} \mathbb{A}^{\infty}\right)^{\times} / \mathbb{K}_{I}^{\prime} .
$$

Let $\Sigma_{\lim , \wp}$ denote the profinite set $\lim _{I} \Sigma_{I, \wp}$ where $I$ runs over the non-zero ideals $I \subset A$ such that $\operatorname{Spec}(A / I)$ contains two or more closed points and that $I$ is prime to $\wp$. Passing to the projective limit we have an isomorphism

$$
\Sigma_{\lim , \wp} \cong B^{\times} \backslash\left(B \otimes_{k} \mathbb{A}^{\infty}\right)^{\times} /\left(\mathcal{O} \otimes_{A} A_{\wp}\right)^{\times}
$$

of sets.

Drinfeld $[\mathrm{Dr} 1, \S 5, \mathrm{D})]$ introduced the right action of the group $\mathrm{GL}_{d}\left(\mathbb{A}^{\infty}\right)$ on the projective limit $\lim _{I} M_{I}^{d}$, where $I$ runs over the non-zero ideals $I \subset A$ (we note that this projective limit exists in the category of schemes over Spec $A$ since, when $\operatorname{Spec}(A / I)$ contains two or more closed points, every $M_{I}^{d}$ is an affine scheme). We restrict our attention to the case where $d=2$; we have a continuous right action of the group $\mathrm{GL}_{2}\left(\mathbb{A}^{\infty}\right)$ on the set $\Sigma_{\lim , \wp}$.

We let $\mathbb{A}^{\infty, \wp}$ denote the prime-to- $\wp$-part of $\mathbb{A}^{\infty}$. In this paper, we often restrict our attention to the subgroup $\mathrm{GL}_{2}\left(\mathbb{A}^{\infty, \wp}\right) \subset \mathrm{GL}_{2}\left(\mathbb{A}^{\infty}\right)$. Recall that in Section 5.2.2 we have obtained an isomorphism $\eta: \mathcal{O} \otimes_{A} \widehat{A}^{\prime} \cong \operatorname{Mat}_{2}\left(\widehat{A}^{\prime}\right)$, where $\widehat{A}^{\prime}=\prod_{\wp^{\prime} \neq \infty, \wp} A_{\wp^{\prime}}$ is the prime-to- $\wp-$-part of $\widehat{A}$, from the fixed isomorphism $\xi$. This induces an isomor$\operatorname{phism}\left(B \otimes_{k} \mathbb{A}^{\infty, \wp}\right)^{\times} \cong \mathrm{GL}_{2}\left(\mathbb{A}^{\infty, \wp}\right)$. 
Proposition 5.4. The action of $\mathrm{GL}_{2}\left(\mathbb{A}^{\infty, \wp}\right)$ on the left hand side of (5.4) coincides with the action of $\left(B \otimes_{k} \mathbb{A}^{\infty, \wp}\right)^{\times}$under the isomorphism $\left(B \otimes_{k} \mathbb{A}^{\infty, \wp}\right)^{\times} \cong \mathrm{GL}_{2}\left(\mathbb{A}^{\infty, \wp}\right)$ above.

Proof. This follows immediately from the definition of the action of the group $\mathrm{GL}_{2}\left(\mathbb{A}^{\infty, \wp}\right)$ on $\lim _{I} M_{I}$ and Lemma 5.5 below.

Lemma 5.5. Let $E$ be a supersingular Drinfeld module of rank two over $\operatorname{Spec} \overline{\kappa(\wp)}$. Let $I \subset A$ be a non-zero ideal which is prime to $\wp$. Let $M_{0}$ be as in Proposition 5.1 and fix an isomorphism $\xi:(k / A)^{\prime \oplus 2} \cong M_{0}$ of $A$-modules.

(1) Let $g$ be an element in $\operatorname{Mat}_{2}(A / I)$. We also denote by $g$ the endomorphism of $\left(I^{-1} / A\right)^{\oplus 2}$ supplied by the left multiplication by $g$. Let $X \subset\left(I^{-1} / A\right)^{\oplus 2}$ denote the kernel of $g$. Let $I^{\prime} \subset A$ be an ideal containing $I$ such that $\left(I^{\prime-1} / A\right)^{\oplus 2} \subset g\left(I^{-1} / A\right)^{\oplus 2} \subset\left(I^{-1} / A\right)^{\oplus 2}$. Let $\varphi:\left(I^{-1} / A\right)^{\oplus 2} \cong E[I]$ be a level $I$ structure on $E$. Let $E^{\prime}$ be the quotient of $E$ by $\varphi(X)$ and let $f: E \rightarrow E^{\prime}$ be the canonical isogeny. Let $\eta_{I}: \operatorname{Mat}_{2}(A / I) \cong \mathcal{O} / I \mathcal{O}$ be the isomorphism induced by the isomorphism $\eta$. Then the image of the composite $\mathcal{F}_{E_{0}}\left(E^{\prime}\right) \stackrel{\mathcal{F}_{E_{0}}(f)}{\longrightarrow} \mathcal{F}_{E_{0}}(E) \stackrel{\Phi_{E_{0}, \xi}(\varphi)}{\longrightarrow} \mathcal{O} / I \mathcal{O}$ is equal to the image of the map $\mathcal{O} / I \mathcal{O} \rightarrow \mathcal{O} / I \mathcal{O}$ supplied by the right multiplication by $\eta_{I}(g)$.

(2) Let the notation be as in (1). Let $\varphi^{\prime}:\left(I^{\prime-1} / A\right)^{\oplus 2} \cong E^{\prime}\left[I^{\prime}\right]$ be the unique level $I^{\prime}$-structure on $E^{\prime}$ satisfying the following property: if $a \in\left(I^{-1} / A\right)^{\oplus 2}$ satisfies $g a \in\left(I^{\prime-1} / A\right)^{\oplus 2}$, then $\varphi^{\prime}(g a)$ is equal to the image of $\varphi(a)$ under the map $E[I] \rightarrow E^{\prime}[I]$ induced by $f$. Then the map $\Phi_{E_{0}, \xi}\left(\varphi^{\prime}\right): \mathcal{F}_{E_{0}}\left(E^{\prime}\right) \rightarrow$ $\mathcal{O} / I^{\prime} \mathcal{O}$ is characterized by the following property: if $b \in \mathcal{F}_{E_{0}}\left(E^{\prime}\right)$ and if we take an element $c \in \mathcal{O} / I \mathcal{O}$ such that $\left(\Phi_{E_{0}, \xi}(\varphi)\right)\left(\mathcal{F}_{E_{0}}(f)(b)\right)=c \eta_{I}(g)$, then $\left(\Phi_{E_{0}, \xi}(\varphi)\right)(b)$ is equal to the image of $c$ under the natural projection $\mathcal{O} / I \mathcal{O} \rightarrow \mathcal{O} / I^{\prime} \mathcal{O}$.

Proof. Let $G$ denote the image of $E[I] \subset E$ under the isogeny $f: E \rightarrow E^{\prime}$ in the category of schemes of $A$-modules over Spec $\overline{\kappa(\wp)}$. Then $G$ contains $E^{\prime}\left[I^{\prime}\right]$ and the isogeny $f$ induces an isomorphism $f_{I}: E[I] / \varphi(X) \cong G$. We have the commutative diagram

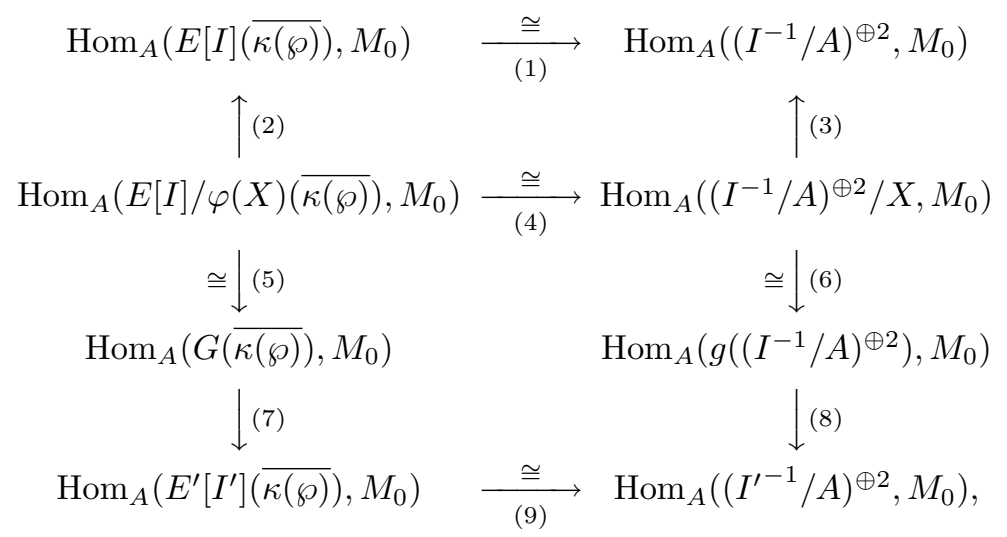

where the maps are defined as follows:

- The maps (1) and (4) are the isomorphisms induced by $\varphi$.

- The map (2) (resp. (3)) is the injective homomorphism induced by the canonical surjection $E[I] \rightarrow E[I] / \varphi(X)$ (resp. $\left.\left(I^{-1} / A\right)^{\oplus 2} \rightarrow\left(I^{-1} / A\right)^{\oplus 2} / X\right)$. 
- The map (5) (resp. (6)) is the isomorphism induced by the isomorphism $f_{I}$ (resp. the isomorphism $\left(I^{-1} / A\right)^{\oplus 2} / X \cong g\left(\left(I^{-1} / A\right)^{\oplus 2}\right)$ supplied by the left multiplication by $g$ ).

- The map (7) (resp. (8)) is the homomorphism induced by the inclusion $E^{\prime}\left[I^{\prime}\right] \subset G$ (resp. $\left.\left(I^{\prime-1} / A\right)^{\oplus 2} \subset g\left(\left(I^{-1} / A\right)^{\oplus 2}\right)\right)$.

- The map (9) is the isomorphism induced by $\varphi^{\prime}$.

It is easily seen from the definition of the isomorphism $\eta_{I}$ that the image of the injection $\operatorname{Hom}_{A}\left(\left(I^{-1} / A\right)^{\oplus 2} / X, M_{0}\right) \hookrightarrow \operatorname{Hom}_{A}\left(\left(I^{-1} / A\right)^{\oplus 2}, M_{0}\right)$ maps isomorphically onto the image $(\mathcal{O} / I \mathcal{O}) \eta_{I}(g)$ of the endomorphism $\mathcal{O} / I \mathcal{O} \rightarrow \mathcal{O} / I \mathcal{O}$ supplied by the right multiplication by $\eta_{I}(g)$ under the isomorphism $\mathcal{O} / I \mathcal{O} \cong \operatorname{Hom}_{A}\left(\left(I^{-1} / A\right)^{\oplus 2}, M_{0}\right)$ in (5.3). Moreover we have the commutative diagram

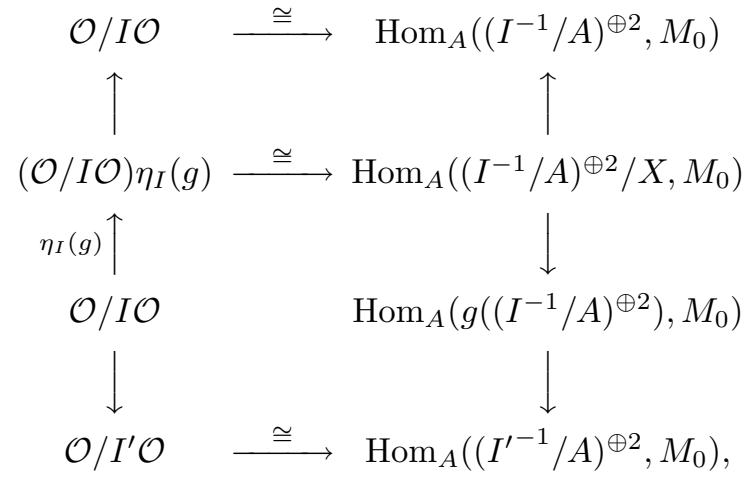

where the maps are defined as follows:

- The left vertical map at the top is the inclusion.

- The left vertical map in the middle is the map supplied by the right multiplication by $\eta_{I}(g)$.

- The left vertical map at the bottom is the canonical surjection $\mathcal{O} / I \mathcal{O} \rightarrow$ $\mathcal{O} / I^{\prime} \mathcal{O}$.

- The right vertical maps are the same as those in the diagram (5.5).

- The horizontal map at the top is the isomorphism (5.3).

- The horizontal map in the middle is the isomorphism induced by the isomorphism (5.3).

- The horizontal map at the bottom is the isomorphism (5.3) with $I$ replaced by $I^{\prime}$.

Hence the claims follow from the definition of the map $\Phi_{E_{0}, \xi}(\varphi)$.

\section{Bad REDuction of Drinfeld modular CuRVES}

The results in this section (to be used in Section 7) are Lemmas 6.4 and 6.5. The reduction modulo $\wp$ of the Drinfeld modular curve of level $I \wp^{n}$ is described. This can be regarded as the Drinfeld modular analogue of [Ka-Ma, p.427, Theorem 13.7.6]. The method is classical. We use the Serre-Tate theory and exotic Igusa curves. The picture of the bad reduction is the same as in [Ka-Ma, p.395].

In Section 6.1, we give a description of the Drinfeld modular curve at a good prime using the determinant morphism from Section 4.4. Lemma 6.1 says that $M_{I / \kappa(\wp)}^{1}$ (which is a spectrum of a field and is an analogue of the reduction $\bmod p$ 
of the ring of cyclotomic integers $\left.\mathbb{Z}\left[\zeta_{n}\right]\right)$ is the field of constants of $M_{I}^{2} \times_{M_{I}^{1}} M_{I / \kappa(\wp)}^{1}$, and the structure morphism is smooth.

The local study of the moduli using Serre-Tate theory is due to Drinfeld [Dr1]. We give the details in Sections 6.2.1 and 6.2.2. In Section 6.2.3, we express the set of connected components and of cusps of the reduction modulo $\wp$ as a coset space. The modular curves considered in Section 6.3 are the analogues of the exotic Igusa curves in the elliptic modular case [Ka-Ma, p.385]. The main results of this section (Lemmas 6.4 and 6.5) are proved in Section 6.3 and 6.4.

We mention that the description of deformation spaces in the case of elliptic $\mathcal{D}$-sheaves is found in $[\mathrm{Bo}]$. He treats the higher dimensional cases as well.

6.1. Throughout this section we will fix a non-zero prime ideal $\wp \subset A$. We denote by $A_{\wp}$ the $\wp$-adic completion of $A$. Let $\kappa(\wp)$ denote the residue field at $\wp$ of $A$. We fix an algebraic closure $\overline{\kappa(\wp)}$ of $\kappa(\wp)$.

Let $I \subset A$ be a non-zero ideal such that $\operatorname{Spec}(A / I)$ contains two or more closed points. For an $A$-algebra $L$ we write $M_{I / L}^{d}$ for $M_{I}^{d} \times_{\operatorname{Spec} A} \operatorname{Spec} L$. Let $w: M_{I}^{2} \rightarrow M_{I}^{1}$ be the determinant morphism on $M_{I}^{2}$ (defined in Section 4.4). Let us consider the following cartesian diagram:

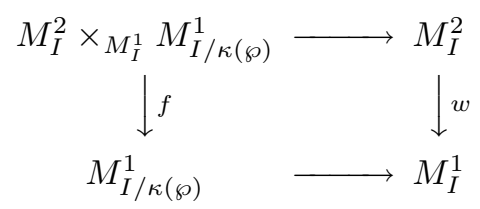

where the morphism at the bottom is the canonical closed immersion.

Lemma 6.1. Suppose that $I$ is prime to $\wp$. Then

(1) The morphism $f$ is smooth.

(2) The fibers of $f$ are geometrically connected.

Proof. Let $g$ be the structure morphism $M_{I}^{1} \rightarrow$ Spec $A$. Then the composite $w \circ g$ is the structure morphism $M_{I}^{2} \rightarrow \operatorname{Spec} A$ hence is smooth outside $\operatorname{Spec}(A / I)$ by Lemma $4.1(2)$. The pullback by $\operatorname{Spec} \kappa(\wp) \rightarrow \operatorname{Spec} A$ of the morphisms $w \circ g$ and $g$ are then smooth, hence so is $f$ which is the pullback of $w$. The claim (1) follows.

The compactification $\bar{M}_{I}^{2}$ of $M_{I}^{2}$, constructed by Drinfeld [Dr1, 99 , p. 585] (see also [Le] for the details), is a regular scheme which is proper over $\operatorname{Spec} A$. Since the generic fiber $\bar{M}_{I}^{2} \times_{\text {Spec } A}$ Spec $k$ is the smooth compactification of the curve $M_{I / k}^{2}$, the determinant morphism $M_{I}^{2} \rightarrow M_{I}^{1}$ gives a morphism $\bar{M}_{I}^{2} \times_{\operatorname{Spec} A} \operatorname{Spec} k \rightarrow M_{I / k}^{1}$. This morphism extends uniquely to a morphism $\bar{w}: \bar{M}_{I}^{2} \rightarrow M_{I}^{1}$ by [EGAII, (6.3.9), p. 119], since $\bar{M}_{I}^{2}$ is regular and $M_{I}^{1}$ equals the integral closure of $A$ in $M_{I / k}^{1}$ (by Lemma 4.2). We know from [Dr1, Proposition 9.3(3), p. 587] and [Dr1, §9 A), p. 585] that the boundary $\bar{M}_{I}^{2} \backslash M_{I}^{2}$ is the disjoint union of a finite number of copies of $M_{I}^{1}$. Let us write $M_{I}^{1}=\operatorname{Spec} R$. The formal completion of $\bar{M}_{I}^{2}$ along any of the components of $\bar{M}_{I}^{2} \backslash M_{I}^{2}$ is isomorphic to $\operatorname{Spf} R[[t]]$ (loc. cit., see also [Le, Chapter 5, Theorem 1.10, p. 72] and the preceding remark). Hence it suffices to prove that each fiber of $\bar{w}$ is geometrically connected. The morphism $\bar{w}$ is proper, since $\bar{M}_{I}^{2} \rightarrow \operatorname{Spec} A$ is proper. From the rigid analytic description of the determinant morphism $w$ given in Proposition 4.5, it follows that the $M_{I / k}^{1}$-scheme 
$M_{I / k}^{2}$ is geometrically connected (note that, by Lemma 4.2, the scheme $M_{I / k}^{1}$ is the spectrum of a field). This implies that the $M_{I / k}^{1}$-scheme $\bar{M}_{I / k}^{2}$ is also geometrically connected, and in particular $H^{0}\left(\bar{M}_{I / k}^{2}, \mathcal{O}_{\bar{M}_{I / k}^{2}}\right)=H^{0}\left(M_{I / k}^{1}, \mathcal{O}_{M_{I / k}^{1}}\right)$. We can then apply [EGAIII1, Corollaire (4.3.12), p. 134] to obtain the claim (2).

6.2. Local description of $M_{I / \overline{\kappa(\wp)}}^{2}$. Let $q_{\wp}$ denote the cardinality of $\kappa(\wp)$. We fix a prime element $\pi \in A_{\wp}$. The canonical projection $A_{\wp} \rightarrow \kappa(\wp)$ has a unique left inverse $\kappa(\wp) \rightarrow A_{\wp}$, which identifies $A_{\wp}$ with the ring $\kappa(\wp)[[\pi]]$ of formal power series.

6.2.1. Universal deformation space of formal $A_{\wp}$-modules over $\overline{\kappa(\wp)}$ with a Drinfeld level structure: the height one case. We define a formal $A_{\wp}$-module $\widehat{F}_{1}$ over $A_{\wp}$ as follows. As a formal group, $\widehat{F}_{1}=\widehat{\mathbb{G}}_{a}$. The action of $a \in \kappa(\wp) \subset A_{\wp}$ on $\widehat{F}_{1}$ is given by the power series $a X$, and the action of $\pi$ is given by the power series $f_{1}(X)=\pi X+X^{q_{\wp}}$. We put $F_{1}=\widehat{F}_{1} \otimes_{A_{\wp}} \kappa(\wp)$. Then $F_{1}$ is a formal $A_{\wp}$-module of height one over $\kappa(\wp)$. By [Dr1, Proposition 1.6, p. 566], any formal $A_{\wp}$-module over $\overline{\kappa(\wp)}$ of height one is isomorphic to $F_{1} \widehat{\otimes}_{\kappa(\wp)} \overline{\kappa(\wp)}$. Let $\widehat{A}_{\wp}^{\text {ur }}$ denote the completion of the strict henselization of $A_{\wp}$. Then it can be checked easily that the formal $A_{\wp}$-module $\widehat{F}_{1} \widehat{\otimes}_{A_{\wp}} \widehat{A}_{\wp}^{\text {ur }}$ is the universal deformation of $F_{1} \widehat{\otimes}_{\kappa(\wp)} \overline{\kappa(\wp)}$.

Let $D_{1, n}$ be the universal deformation ring of $F_{1} \widehat{\otimes}_{\kappa(\wp)} \overline{\kappa(\wp)}$. For $r \geq 0$ let $g_{1, r}=$ $f_{1} \circ \cdots \circ f_{1}$ be the $r$-th iteration of $f_{1}$. Then for $n \geq 1$ the ring $D_{1, n}$ is isomorphic to $\widehat{A}_{\wp}^{\text {ur }}[[x]] /\left(g_{1, n}(x) / g_{1, n-1}(x)\right)$ (cf. [Dr1, proof of Proposition 4.3, p. 573]). Let us note that the reduction modulo $\pi$ of $D_{1, n}$ is isomorphic to $\overline{\kappa(\wp)}[[x]] /\left(x^{q_{\wp}^{n}-q_{\wp}^{n-1}}\right)$.

Let $I \subset A$ be a non-zero ideal, which is prime to $\wp$, such that $\operatorname{Spec}(A / I)$ contains two or more closed points. We set $I_{n}=I \wp^{n}$. Let $\mathcal{P}_{n}$ denote the set of $A$-submodules of $\left(\wp^{-n} / A\right)^{\oplus 2}$ which is free of rank one over $A / \wp^{n}$.

Let $\bar{z} \in M_{I / \overline{\kappa(\wp)}}^{2}(\overline{\kappa(\wp)})$ be a $\overline{\kappa(\wp)}$-rational point which corresponds to an ordinary Drinfeld module $E_{\bar{z}}$ over $\overline{\kappa(\wp)}$. That is, if we regard $\bar{z}$ as a morphism $\operatorname{Spec} \overline{\kappa(\wp)} \rightarrow M_{I / \overline{\kappa(\wp)}}^{2}$, then $E_{\bar{z}}$ is the pullback by $\bar{z}$ of the universal Drinfeld module over $M_{I / \overline{\kappa(\wp)}}^{2}$. Let $M_{I_{n} / \overline{\kappa(\wp)}, \bar{z}}^{2}=\operatorname{Spec} \overline{\kappa(\wp)} \times_{M_{I / \overline{\kappa(\wp)}}^{2}} M_{I_{n} / \overline{\kappa(\wp)}}^{2}$ be the fiber product of $\bar{z}$ and the canonical morphism $M_{I_{n} / \overline{\kappa(\wp)}}^{2} \rightarrow M_{I / \overline{\kappa(\wp)}}^{2}$. The following description of the formal completion $\widehat{M}_{I_{n} / \overline{\kappa(\wp)}, \bar{z}}^{2}$ of $M_{I_{n} / \overline{\kappa(\wp)}}^{2}$ along $M_{I_{n} / \overline{\kappa(\wp)}, \bar{z}}^{2}$ is due to Drinfeld [Dr1, $\S 5$ C) Proposition 5.4, p. 576]. If we fix an isomorphism of the formal $A_{\wp}$-module associated to $E_{\bar{z}}$ to $F_{1}$, then $\widehat{M}_{I_{n} / \overline{\kappa(\wp)}, \bar{z}}^{2}$ is canonically isomorphic to the disjoint union $\coprod_{Q \in \mathcal{P}_{n}} \operatorname{Spf}\left(D_{1, n} /(\pi)[[y]]\right)$ of copies of $\operatorname{Spf}\left(D_{1, n} /(\pi)[[y]]\right) \cong$ $\operatorname{Spf} \overline{\kappa(\wp)}[[x, y]] /\left(x^{q_{\wp}^{n}}-q_{\wp}^{n-1}\right)$.

6.2.2. Universal deformation space of formal $A_{\wp}$-modules over $\overline{\kappa(\wp)}$ with a Drinfeld level structure: the height two case. We define a formal $A_{\wp}$-module $\widehat{F}_{2}$ over the ring $A_{\wp}[[t]]$ of formal power series as follows. As a formal group, $\widehat{F}_{2}=\widehat{\mathbb{G}}_{a}$. The action of $a \in \kappa(\wp) \subset A_{\wp}$ on $\widehat{F}_{2}$ is given by the power series $a X$, and the action of $\pi$ is given by the power series $f_{2}(X)=\pi X+t X^{q_{\wp}}+X^{q_{\wp}^{2}}$. We put $F_{2}=\widehat{F}_{2} \otimes_{A_{\wp}[[t]]} \kappa(\wp)$. Then $F_{2}$ is a formal $A_{\wp}$-module of height two over $\kappa(\wp)$. By [Dr1, Proposition 1.6, p. 566], any formal $A_{\wp}$-module over $\overline{\kappa(\wp)}$ of height two is isomorphic to $F_{2} \widehat{\otimes}_{\kappa(\wp)} \overline{\kappa(\wp)}$. 
Lemma 6.2. The formal $A_{\wp}$-module $\widehat{F}_{2} \widehat{\otimes}_{A_{\wp}[[t]]} \widehat{A}_{\wp}^{\mathrm{ur}}[[t]]$ is the universal deformation of $F_{2} \widehat{\otimes}_{\kappa(\wp)} \overline{\kappa(\wp)}$.

Proof. By [Dr1, Proposition 4.2, p. 570], the universal deformation ring of the formal $A_{\wp}$-module $F_{2}$ equals $\widehat{A}_{\wp}^{\text {ur }}\left[\left[t_{1}\right]\right]$. The universality gives the homomorphism $\varphi: \widehat{A}_{\wp}^{\mathrm{ur}}\left[\left[t_{1}\right]\right] \rightarrow \widehat{A}_{\wp}^{\mathrm{ur}}[[t]]$. Since the coefficient of $X$ in $f_{2}$ is $\pi$, the homomorphism $\varphi$ is a homomorphism of $\widehat{A}_{\wp}^{\text {ur }}$-algebra. It suffices to prove that the image $\varphi\left(t_{1}\right)$ is a topological generator of $\widehat{A}_{\wp}^{\text {ur }}[[t]]$. Let $\Lambda_{A_{\wp}}$ (resp. $\left.\widetilde{\Lambda}_{A_{\wp}}\right)$ be the graded $\operatorname{ring} \Lambda_{O}$ (resp. $\left.\widetilde{\Lambda}_{O}\right)$ defined in [Dr1, $\S 1$, p. 563] for $O=A_{\wp}$. In [Dr1, Proposition 1.4, p. 565], Drinfeld shows that $\Lambda_{A_{\wp}}$ equals the polynomial ring with generators $g_{1}, g_{2}, \cdots \in$ $\Lambda_{A_{\wp}}$ such that $\operatorname{deg} g_{i}=i$. In view of his construction of the $g_{j}$ 's, we may choose $g_{q_{\wp}-1}$ in such a way that the image of $g_{q_{\wp}-1}$ in $\widetilde{\Lambda}_{A_{\wp}}$ equals the element $u$ in [Dr1, Proposition $1.3(2)$, p. 564] for $n=q_{\wp}$. By the construction of $\widehat{A}_{\wp}^{\mathrm{ur}}\left[\left[t_{1}\right]\right]$, there exists a canonical ring homomorphism $\Lambda_{A_{\wp}} \rightarrow \widehat{A}_{\wp}^{\text {ur }}\left[\left[t_{1}\right]\right]$ such that the image of $g_{q_{\wp}-1}$ equals $t_{1}$. This implies that $\varphi\left(t_{1}\right)=-t /\left(1-\pi^{q_{\wp}-1}\right)+O\left(t^{2}\right)$.

The following description of the universal deformation ring $D_{2, n}$ of $F_{2} \widehat{\otimes}_{\kappa(\wp)} \overline{\kappa(\wp)}$ of level $n$ is due to Drinfeld [Dr1, proof of Proposition 4.3, p. 573]. For $n=1$, it is given by

$$
D_{2,1} \cong \widehat{A}_{\wp}^{\mathrm{ur}}\left[\left[t, \theta_{1}, \theta_{2}\right]\right] /\left(\pi+t \theta_{1}^{q_{\wp}-1}+\theta_{1}^{q_{\wp}^{2}-1}, t+\frac{\theta_{2}^{q_{\wp}^{2}-1}-\theta_{1}^{q_{\wp}^{2}-1}}{\theta_{2}^{q_{\wp}-1}-\theta_{1}^{q_{\wp}-1}}\right) .
$$

For $r \geq 0$ let $g_{2, r}=f_{2} \circ \cdots \circ f_{2}$ be the $r$-th iteration of $f_{2}$. Then for $n \geq 1$ the ring $D_{2, n}$ is isomorphic to

$$
D_{2,1}\left[\left[y_{1}, y_{2}\right]\right] /\left(g_{2, n-1}\left(y_{1}\right)-\theta_{1}, g_{2, n-1}\left(y_{2}\right)-\theta_{2}\right) \text {. }
$$

Let $\bar{z} \in M_{I / \overline{\kappa(\wp)}}^{2}(\overline{\kappa(\wp)})$ be a $\overline{\kappa(\wp)}$-rational point which corresponds to a supersingular Drinfeld module $E_{\bar{z}}$ over $\overline{\kappa(\wp)}$. That is, if we regard $\bar{z}$ as a morphism $\operatorname{Spec} \overline{\kappa(\wp)} \rightarrow M_{I / \overline{\kappa(\wp)}}^{2}$, then $E_{\bar{z}}$ is the pullback by $\bar{z}$ of the universal Drinfeld module over $M_{I / \overline{\kappa(\wp)}}^{2}$. Let $M_{I_{n} / \overline{\kappa(\wp)}, \bar{z}}^{2}=\operatorname{Spec} \overline{\kappa(\wp)} \times_{M_{I / \overline{\kappa(\wp)}}^{2}} M_{I_{n} / \overline{\kappa(\wp)}}^{2}$ be the fiber product of $\bar{z}$ and the canonical morphism $M_{I_{n} / \overline{\kappa(\wp)}}^{2} \rightarrow M_{I / \overline{\kappa(\wp)}}^{2}$. The following description of the formal completion $\widehat{M}_{I_{n} / \overline{\kappa(\wp)}, \bar{z}}^{2}$ of $M_{I_{n} / \overline{\kappa(\wp)}}^{2}$ along the fiber $M_{I_{n} / \overline{\kappa(\wp)}, \bar{z}}^{2}$ of $\bar{z}$ is due to Drinfeld [Dr1, $\S 5$ C) Proposition 5.4, p. 576]. If we fix an isomorphism of the formal $A_{\wp}$-module associated to $E_{\bar{z}}$ to $F_{2}$, then $\widehat{M}_{I_{n} / \overline{\kappa(\wp)}, \bar{z}}^{2}$ is canonically isomorphic to $\operatorname{Spf}\left(D_{2, n} /(\pi)\right)$.

6.2.3. Let $I \subset A$ be a non-zero ideal, which is prime to $\wp$, $\operatorname{such}$ that $\operatorname{Spec}(A / I)$ contains two or more closed points. A closed point in $M_{I / \kappa(\wp)}^{2}$ is called ordinary (resp. supersingular) if the corresponding Drinfeld module is ordinary (resp. supersingular). We denote by $\Sigma_{I, \wp}$ the set of supersingular points of $M_{I / \overline{\kappa(\wp)}}^{2}$. Let $S_{I, \wp}$ denote the set of connected components of $M_{I / \overline{\kappa(\wp)}}^{2}$. There is a canonical map $\Sigma_{I, \wp} \rightarrow S_{I, \wp}$ sending a point to the connected component to which it belongs. 
We fix an algebraic closure $\bar{k}_{\infty}$ of $k_{\infty}$. For an integer $n \geq 0$, let $\operatorname{Cusp}_{I, \wp^{n}}$ denote the set of cusps of $M_{I \wp^{n} / \bar{k}_{\infty}}^{2}$, that is, the set of the closed points of the complement of $M_{I_{\wp^{n}} / \bar{k}_{\infty}}^{2}$ in its smooth compactification.

Let $S_{\lim , \wp}\left(\right.$ resp. $\left.\operatorname{Cusp}_{\lim , \wp^{n}}\right)$ denote the profinite set $\lim _{I} S_{I, \wp},\left(\right.$ resp. $\left.\lim _{I} \operatorname{Cusp}_{I, \wp^{n}}\right)$ where $I$ runs over the non-zero ideals $I \subset A$ such that such that $\operatorname{Spec}(A / I)$ contains two or more closed points and that $I$ is prime to $\wp$. There is a canonical morphism $\Sigma_{\lim , \wp} \rightarrow S_{\lim , \wp}$ of profinite sets.

The right action the group $\mathrm{GL}_{d}\left(\mathbb{A}^{\infty}\right)$ on the projective limit $\lim _{I} M_{I}^{d}$, where $I$ runs over the non-zero ideals $I \subset A$ gives a continuous right action of the group $\mathrm{GL}_{2}\left(\mathbb{A}^{\infty}\right)$ on the set $S_{\lim , \wp}$, and the canonical morphism $\Sigma_{\lim , \wp} \rightarrow S_{\lim , \wp}$ described in the previous paragraph is $\mathrm{GL}_{2}\left(\mathbb{A}^{\infty, \wp)}\right)$-equivariant.

Lemma 6.3. Let $T \subset \mathrm{GL}_{2}$ (resp. $N \subset \mathrm{GL}_{2}$ ) denote the diagonal torus (resp. the subgroup of upper triangular unipotent matrices) of $\mathrm{GL}_{2}$.

(1) There are canonical isomorphisms

$$
\begin{aligned}
S_{I, \wp} & \cong k^{\times} \backslash\left(\mathbb{A}^{\infty}\right)^{\times} / \operatorname{Ker}\left(\widehat{A}^{\times} \rightarrow(A / I)^{\times}\right), \\
\operatorname{Cusp}_{I, \wp^{n}} & \cong T(k) N\left(\mathbb{A}^{\infty}\right) \backslash \operatorname{GL}_{2}\left(\mathbb{A}^{\infty}\right) / \mathbb{K}_{I \wp^{n}} .
\end{aligned}
$$

(2) There are canonical isomorphisms

$$
\begin{aligned}
S_{\lim , \wp} & \cong k^{\times} \backslash\left(\mathbb{A}^{\infty}\right)^{\times} / A_{\wp}^{\times}, \\
\operatorname{Cusp}_{\lim , \wp^{n}} & \cong T(k) N\left(\mathbb{A}^{\infty}\right) \backslash \mathrm{GL}_{2}\left(\mathbb{A}^{\infty}\right) /\left(1+\wp^{n} \operatorname{Mat}_{2}\left(A_{\wp}\right)\right)
\end{aligned}
$$

of $\mathrm{GL}_{2}\left(\mathbb{A}^{\infty, \wp}\right)$-sets. Here $\mathrm{GL}_{2}\left(\mathbb{A}^{\infty, \wp}\right)$ acts on $\left(\mathbb{A}^{\infty}\right)^{\times} / A_{\wp}^{\times}$via the determinant det : $\mathrm{GL}_{2}\left(\mathbb{A}^{\infty, \wp}\right) \rightarrow\left(\mathbb{A}^{\infty, \wp}\right)^{\times}$.

(Recall the definition of $\mathbb{K}_{*}$ from Section 4.3.2.)

Proof. The set of cusps $\operatorname{Cusp}_{I, \wp^{n}}$ is isomorphic to the double coset

$$
\mathrm{GL}_{2}(k) \backslash\left(\mathrm{GL}_{2}\left(\mathbb{A}^{\infty}\right) / \mathbb{K}_{I_{\wp}{ }^{n}} \times \mathbb{P}^{1}(k)\right) \cong T(k) N(k) \backslash \mathrm{GL}_{2}\left(\mathbb{A}^{\infty}\right) / \mathbb{K}_{I_{\wp^{n}}}
$$

(see, for example, [Gek2, (1.2.5), p. 354]). The claim for the cusps follows since $N(k)$ is dense in $N\left(\mathbb{A}^{\infty}\right)$.

We prove the claim for $S_{I, \wp}$. By Lemma 6.1, the set $S_{I, \wp}$ is canonically isomorphic to the set of connected components of $M_{I / \overline{\kappa(\wp)}}^{1}$. Since $M_{I}^{1} \times_{\operatorname{Spec} A} U_{I}$ is finite étale over $U_{I}$, it is also isomorphic to the set of connected components of $M_{I}^{1} \times_{\operatorname{Spec} A} \operatorname{Spec} \bar{k}_{\infty}$ By the adelic description (4.2) of $\left(M_{I}^{1} \times_{\operatorname{Spec} A} \operatorname{Spec} k_{\wp}\right)^{\text {an }}$, it is also isomorphic to the double coset

$$
k^{\times} \backslash\left(\mathbb{A}^{\infty}\right)^{\times} / \operatorname{Ker}\left(\widehat{A}^{\times} \rightarrow(A / I)^{\times}\right) .
$$

This proves (1). Taking the projective limit, we have (2).

6.3. Fix an integer $n \geq 1$. For a non-zero ideal $I \subset A$ such that $\operatorname{Spec}(A / I)$ contains two or more closed points and that $I$ is prime to $\wp$, we put $I_{n}=I \wp^{n}$. Let us describe the reduction at $\wp$ of the moduli scheme $M_{I_{n}}^{2}$.

For $Q \in \mathcal{P}_{n}$ (defined in Section 6.2.1), we let $M_{I_{n} / \kappa(\wp), Q}^{2} \subset M_{I_{n} / \kappa(\wp)}^{2}$ denote the closed subscheme which classifies the Drinfeld modules of rank two with a Drinfeld level $I_{n}$ structure $\phi$ such that $Q \subset \operatorname{Ker} \phi$. This is an analogue of the Igusa curve. We set $M_{I_{n} / \overline{\kappa(\wp)}, Q}^{2}=M_{I_{n} / \kappa(\wp), Q}^{2} \times_{\operatorname{Spec} \kappa(\wp)} \operatorname{Spec} \overline{\kappa(\wp)}$. 
Lemma 6.4. (1) The underlying topological space of $M_{I_{n} / \kappa(\wp)}^{2}$ equals the union of the underlying topological spaces of the $M_{I_{n} / \kappa(\wp), Q}^{2}$ 's.

(2) For $Q, Q^{\prime} \in \mathcal{P}_{n}$ with $Q \neq Q^{\prime}$, the scheme $M_{I_{n} / \kappa(\wp), Q}^{2}$ intersects $M_{I_{n} / \kappa(\wp), Q^{\prime}}^{2}$ only at supersingular points.

(3) The scheme $M_{I_{n} / \kappa(\wp), Q}^{2}$ is a smooth curve over Spec $\kappa(\wp)$.

(4) The morphism $M_{I_{n} / \kappa(\wp), Q}^{2} \rightarrow M_{I / \kappa(\wp)}^{2}$ is finite, flat, surjective, of constant degree and is totally ramified at every supersingular point on $M_{I / \kappa(\wp)}^{2}$. This also induces a bijection between the set of connected components of $M_{I_{n} / \overline{\kappa(\wp)}, Q}^{2}$ and that of $M_{I / \overline{\kappa(\wp)}}^{2}$.

Proof. We will prove the following four statements for every closed point $\bar{x} \in$ $M_{I / \overline{\kappa(\wp)}}^{2}$ :

(1)' The fiber $M_{I_{n} / \overline{\kappa(\wp)}, \bar{x}}^{2}$ of $M_{I_{n} / \overline{\kappa(\wp)}}^{2}$ at $\bar{x}$ is non-empty and is the union of the $M_{I_{n} / \overline{\kappa(\wp)}, Q, \bar{x}}^{2}$ 's.

$(2)^{\prime}$ If $\bar{x}$ is an ordinary point, then $M_{I_{n} / \overline{\kappa(\wp)}, \bar{x}}^{2}$ is the disjoint union of the $M_{I_{n} / \overline{\kappa(\wp)}, Q, \bar{x}}^{2}$ 's.

$(3)^{\prime}$ The scheme $M_{I_{n} / \overline{\kappa(\wp)}, Q}^{2}$ is smooth over Spec $\overline{\kappa(\wp)}$ in a neighborhood of $M_{I_{n} / \overline{\kappa(\wp)}, Q, \bar{x}}^{2}$

$(4)^{\prime}$ If $\bar{x}$ is a supersingular point, then the morphism $M_{I_{n} / \overline{\kappa(\wp)}, Q}^{2} \rightarrow M_{I / \overline{\kappa(\wp)}, Q}^{2}$ is totally ramified at $\bar{x}$, whose ramification index does not depend on the choice of $\bar{x}$.

It is clear that the assertions (1), (2) and (3) follow from $(1)^{\prime},(2)^{\prime}$, and $(3)^{\prime}$. Let us denote by $f$ the morphism $M_{I_{n} / \kappa(\wp), Q}^{2} \rightarrow M_{I / \kappa(\wp)}^{2}$ in the statement of (4). Then $f$ is finite flat since $M_{I \wp^{n}}^{2} \rightarrow M_{I}^{2}$ is finite flat. By (1), we see that $f$ is surjective. The remaining assertions in (4) follow from $(4)^{\prime}$ and the existence of a supersingular point on each connected component of $M_{I / \overline{\kappa(\wp)}}^{2}$.

Let $E \rightarrow \bar{x}$ denote the Drinfeld module of rank two over $\bar{x}$ corresponding to the geometric point $\bar{x}$. Then the $\wp^{n}$-torsion subgroup of the $A$-module $E(\bar{x})$ is a free $A / \wp^{n}$-module of rank $\leq 1$. Hence for any $A$-module homomorphism $\left(I_{n}^{-1} / A\right)^{\oplus 2} \rightarrow$ $E(\bar{x})$, its kernel contains a submodule isomorphic to $A / \wp^{n}$. We regard this as an element $Q \in \mathcal{P}_{n}$. This implies $(1)^{\prime}$.

The group $\mathrm{GL}_{2}\left(A / \wp^{n}\right)$ acts both on $\mathcal{P}_{n}$ and on $M_{I_{n} / \kappa(\wp)}^{2}$. The action of $g \in$ $\mathrm{GL}_{2}\left(A / \wp^{n}\right)$ on $M_{I_{n} / \overline{\kappa(\wp)}}^{2}$ maps $M_{I_{n} / \overline{\kappa(\wp)}, Q}^{2}$ isomorphically onto $M_{I_{n} / \overline{\kappa(\wp)}, g Q}^{2}$. Since the group $\mathrm{GL}_{2}\left(A / \wp^{n}\right)$ acts transitively on $\mathcal{P}_{n} \cong \mathbb{P}^{1}\left(A / \wp^{n}\right)$, all the $M_{I_{n} / \overline{\kappa(\wp)}, Q}^{2}$ 's are isomorphic as schemes over $M_{I / \overline{\kappa(\wp)}}^{2}$. Hence it suffices to show that $(3)^{\prime}$ and $(4)^{\prime}$ hold for $Q=Q_{0}$, where $Q_{0}$ is the second direct summand $\wp^{-n} / A$ of $\left(\wp^{-n} / A\right)^{\oplus 2}$.

It follows from the description in Section 6.2.1 that the completion of the local ring of $M_{I_{n} / \overline{\kappa(\wp)}, Q_{0}}^{2}$ at an ordinary point equals

$$
\left(D_{1, n} \otimes_{\widehat{A}_{\wp}^{\text {ur }}} \overline{\kappa(\wp)}\right)[[y]] /(x) \cong \overline{\kappa(\wp)}[[y]] .
$$

This proves $(2)^{\prime}$.

The claim $(3)^{\prime}$ for an ordinary point $\bar{z}$ immediately follows from the argument in the proof of $(2)^{\prime}$. Suppose that $\bar{z}$ is a supersingular point. It follows from the 
description in Section 6.2.2 that the coordinate ring of the completion of $M_{I_{n} / \overline{\kappa(\wp)}, Q_{0}}^{2}$ along the fiber of $\bar{z}$ equals

$$
\left(D_{2, n} \otimes_{\widehat{A}_{\wp}^{\text {ur }}} \overline{\kappa(\wp)}\right) /\left(y_{2}\right)=\overline{\kappa(\wp)}\left[\left[t, \theta_{1}, y_{1}\right]\right] /\left(t+\theta_{1}^{q_{\wp}^{2}-q_{\wp}}, g_{2, n}\left(y_{1}\right)-\theta_{1}\right) .
$$

From this description, we easily see that the canonical homomorphism $\overline{\kappa(\wp)}\left[\left[y_{1}\right]\right] \rightarrow$ $\left(D_{2, n} \otimes_{\widehat{A}_{\wp}^{\text {ur }}} \overline{\kappa(\wp)}\right) /\left(y_{2}\right)$ is an isomorphism. Hence $M_{I_{n} / \overline{\kappa(\wp)}, Q_{0}}^{2}$ is smooth over Spec $\overline{\kappa(\wp)}$ in a neighborhood of $M_{I_{n} / \overline{\kappa(\wp)}, Q_{0}, \bar{z}}^{2}$, and $M_{I_{n} / \overline{\kappa(\wp)}, Q_{0}}^{2} \rightarrow M_{I / \overline{\kappa(\wp)}}^{2}$ is totally ramified at $\bar{z}$. This proves $(3)^{\prime}$.

Let $\bar{z}$ be a supersingular point. Then, using the notations in the proof of $(3)^{\prime}$, the ring homomorphism from the completion of the local ring of $M_{I / \kappa(\wp)}^{2}$ at $\bar{z}$ to the coordinate ring of the completion of $M_{I_{n} / \overline{\kappa(\wp)}, Q_{0}}^{2}$ along the fiber of $\bar{z}$ is the homomorphism

$$
\overline{\kappa(\wp)}[[t]] \rightarrow\left(D_{2, n} \otimes_{\widehat{A_{\wp}}} \overline{\kappa(\wp)}\right) /\left(y_{2}\right) \cong \overline{\kappa(\wp)}\left[\left[y_{1}\right]\right] .
$$

This homomorphism is totally ramified and its ramification index is independent of the choice of $\bar{z}$. This proves $(4)^{\prime}$.

6.4. Fix a non-zero ideal $I \subset A$, which is prime to $\wp$, such that $\operatorname{Spec}(A / I)$ contains two or more closed points. Let $n \geq 0$ be an integer. We write $I_{n}=I \wp^{n}$. Let us consider the compactification $\bar{M}_{I_{n}}^{2}$ of $M_{I_{n}}^{2}$ constructed by Drinfeld [Dr1, §9, p. 585].

Lemma 6.5. For $Q \in \mathcal{P}_{n}$, let $\bar{M}_{I_{n} / \kappa(\wp), Q}^{2} \subset \bar{M}_{I_{n}}^{2}$ denote the closure of $M_{I_{n} / \kappa(\wp), Q}^{2}$. Then

(1) The scheme $\bar{M}_{I_{n} / \kappa(\wp), Q}^{2}$ is a projective smooth curve over $\kappa(\wp)$.

(2) For $Q^{\prime} \in \mathcal{P}_{n}$ with $Q \neq Q^{\prime}$, the schemes $\bar{M}_{I_{n} / \kappa(\wp), Q}^{2}$ and $\bar{M}_{I_{n} / \kappa(\wp), Q^{\prime}}^{2}$ do not intersect at a point in the boundary $\bar{M}_{I_{n}}^{2} \backslash M_{I_{n}}^{2}$.

Proof. We note that, to prove (1), it suffices to show that $\bar{M}_{I_{n} / \kappa(\wp), Q}^{2}$ is non-singular at the boundary $\bar{M}_{I_{n} / \kappa(\wp), Q}^{2} \backslash M_{I_{n} / \kappa(\wp), Q}^{2}$.

Since $M_{I_{n} / \kappa(\wp), Q}^{2}$ is a smooth curve and $\bar{M}_{I_{n}}^{2}$ is regular, the closure $\bar{M}_{I_{n} / \kappa(\wp), Q}^{2}$ is reduced. Hence both (1) and (2) follow if we prove that $\left(\bar{M}_{I_{n} / \kappa(\wp)}^{2}\right)_{\text {red }}$ is regular at the boundary.

Let us write $M_{I}^{1}=\operatorname{Spec} R$. As noted in the proof of Lemma 6.1, the boundary $\bar{M}_{I_{n}}^{2} \backslash M_{I_{n}}^{2}$ is the disjoint union of a finite number of copies of $\operatorname{Spec} R$, and for each component of $\bar{M}_{I_{n}}^{2} \backslash M_{I_{n}}^{2}$, the coordinate ring of the completion of $\bar{M}_{I_{n}}^{2}$ along it is isomorphic to $R[[t]]$. The claim follows since $\left(R \otimes_{A} \kappa(\wp)[[t]]\right)_{\text {red }}$ is regular.

\section{INTEGRALITY}

The main result of this section is Corollary 7.4. We use this in the form Proposition 7.6 in the proof of Proposition 9.1. This is the only result to be used in other sections.

We give in this section the Drinfeld modular analogue of the result of Beilinson on integrality in the form presented by Schappacher and Scholl [Sc-Sc, Section 7, p. 298-302]. Let us briefly recall (a part of) their result. Let $N \geq 1$ be an integer, which we assume to be divisible by at least two distinct primes. In his work on 
the $K_{2}$ of a modular curve, concerning his conjectures on L-functions, Beilinson constructs what are called Beilinson elements in the rational $K_{2}$ of the compactified modular curves $X(N)$ over $\mathbb{Q}$ where $N$ means the level. The method is, first consider the image of the symbol map $\mathcal{O}(Y(N))^{\times \otimes 2} \rightarrow K_{2}(Y(N))$ for (open) modular curve $Y(N)$, then to use Bloch's method (See the proof of Lemma 9.3; the crucial ingredient is the Drinfeld-Manin theorem on the finiteness of the cuspidal divisor class group.) to obtain elements in $K_{2}(X(N))_{\mathbb{Q}}$. The integrality of elements in $K_{2}(X(N))_{\mathbb{Q}}$ means that they lie in the kernel of the boundary map $K_{2}(X(N))_{\mathbb{Q}} \stackrel{\oplus_{p} \partial_{p}}{\longrightarrow} \oplus_{p} G_{1}\left(X(N)_{p}\right)_{\mathbb{Q}}$ where the subscript $p$ means the reduction modulo $p$ of the regular model over $\mathbb{Z}$.

As the $G_{1}$ (or equivalently $K_{1}$ ) of a projective smooth curve over a finite field is torsion (see Lemma 7.5), we only need to consider those primes $p$ dividing the level $N$. At those primes $p$ dividing the level $N$, one requires the study of the reduction of modular curves (we have presented the Drinfeld modular analogue in Section 6), and some facts from the theory of automorphic forms.

In Section 7.1, we give a basic result (Lemma 7.1), which is an application of the results of previous sections. In Section 7.2 , we give the analogue of $[\mathrm{Sc}-\mathrm{Sc}]^{* * *}$. In Section 7.3, we put the result of this section in the form to be used in later section. This requires some easy computation of the rational $G$-group of a curve over a finite field and some diagram chasing.

Let $I \subsetneq A$ be a non-zero ideal such that $\operatorname{Spec}(A / I)$ contains two or more closed points. For a scheme $X$, we let $\mathcal{O}(X)=H^{0}\left(X, \mathcal{O}_{X}\right)$.

7.1. Let $M$ be an abelian group. Let us recall that we have introduced the two sets $\Sigma_{I, \wp}$ and $S_{I, \wp}$ in Section 6.2.3. We write $M\left[\Sigma_{I, \wp}\right], M\left[S_{I, \wp}\right]$, for the groups of $M$-valued functions on the sets $\Sigma_{I, \wp}, S_{I, \wp}$, respectively. The canonical map $\Sigma_{I, \wp} \rightarrow S_{I, \wp}$ induces a homomorphism $\gamma_{I}: M\left[S_{I, \wp}\right] \rightarrow M\left[\Sigma_{I, \wp}\right]$. We set $\mathcal{C}\left(\Sigma_{\lim , \wp}, M\right)=\lim _{\longrightarrow} M\left[\Sigma_{I, \wp}\right]$ where $I$ runs over all non-zero ideals of $A$ prime to $\wp$ such that $\operatorname{Spec}(A / I)$ contains two or more closed points, and define the abelian groups $\mathcal{C}\left(S_{\lim , \wp}, M\right)$ and $\mathcal{C}\left(\operatorname{Cusp}_{\lim , \wp}, M\right)$, and the homomorphism $\gamma_{\text {lim }}$ in a similar manner. The action of $\mathrm{GL}_{d}\left(\mathbb{A}^{\infty}\right)$ on $\lim _{I} M_{I}^{2}$ which we have recalled in Section 6.2.3 induces actions of the group $\mathrm{GL}_{2}\left(\mathbb{A}^{\infty, \wp}\right)$ on the sets $\mathcal{C}\left(S_{\lim , \wp}, M\right)$ and $\mathcal{C}\left(\operatorname{Cusp}_{\lim , \wp}, M\right)$, and the map $\gamma_{\lim }$ is $\mathrm{GL}_{2}\left(\mathbb{A}^{\infty, \wp}\right)$-equivariant.

Lemma 7.1. The cokernel of $\gamma_{\lim }: \mathcal{C}\left(S_{\lim , \wp}, \mathbb{C}\right) \rightarrow \mathcal{C}\left(\Sigma_{\lim , \wp}, \mathbb{C}\right)$ is a direct sum of irreducible admissible representations of $\mathrm{GL}_{d}\left(\mathbb{A}^{\infty, \wp}\right)$. Each direct summand is cuspidal automorphic, that is, isomorphic to the $\mathbb{A}^{\infty, \wp}$-component of a cuspidal irreducible automorphic representations of $\mathrm{GL}_{d}(\mathbb{A})$.

Proof. By the isomorphisms in Proposition 5.4 and in Lemma 6.3, we can regard the canonical map $\Sigma_{\lim , \wp} \rightarrow S_{\lim , \wp}$ as a $\operatorname{GL}_{2}\left(\mathbb{A}^{\wp, \infty}\right)$-equivariant map

$$
B^{\times} \backslash\left(B \otimes_{k} \mathbb{A}^{\infty}\right)^{\times} /\left(\mathcal{O} \otimes_{A} A_{\wp}\right)^{\times} \rightarrow k^{\times} \backslash\left(\mathbb{A}^{\infty}\right)^{\times} / A_{\wp}^{\times}
$$

where the group $\mathrm{GL}_{2}\left(\mathbb{A}^{\wp, \infty}\right)$ acts on the left hand side via the isomorphism $\left(B \otimes_{k}\right.$ $\left.\mathbb{A}^{\infty, \wp}\right)^{\times} \cong \mathrm{GL}_{2}\left(\mathbb{A}^{\infty, \wp}\right)$ in Section 5.2.2.

Observe that any $\mathrm{GL}_{2}\left(\mathbb{A}^{\wp, \infty}\right)$-equivariant map from $B^{\times} \backslash\left(B \otimes_{k} \mathbb{A}^{\infty}\right)^{\times} /\left(\mathcal{O} \otimes_{A}\right.$ $\left.A_{\wp}\right)^{\times}$to $k^{\times} \backslash\left(\mathbb{A}^{\infty}\right)^{\times} / A_{\wp}^{\times}$equals the composite

$$
B^{\times} \backslash\left(B \otimes_{k} \mathbb{A}^{\infty}\right)^{\times} /\left(\mathcal{O} \otimes_{A} A_{\wp}\right)^{\times} \stackrel{\operatorname{nrd}}{\longrightarrow} k^{\times} \backslash\left(\mathbb{A}^{\infty}\right)^{\times} / A_{\wp}^{\times} \stackrel{\alpha}{\longrightarrow} k^{\times} \backslash\left(\mathbb{A}^{\infty}\right)^{\times} / A_{\wp}^{\times}
$$


for some $\left(\mathbb{A}^{\infty, \wp}\right)^{\times}$-equivariant isomorphism $\alpha$ of sets, where the map nrd is the map induced by the reduced norm $B \rightarrow k$ on $B$. This shows that the image of $\gamma_{\text {lim }}$ is equal to the $\mathbb{C}$-vector space of locally constant $\mathbb{C}$ valued functions $f$ on the set $B^{\times} \backslash\left(B \otimes_{k} \mathbb{A}^{\infty}\right)^{\times} /\left(\mathcal{O} \otimes_{A} A_{\wp}\right)^{\times}$such that $f$ is the pullback of a function on the set $k^{\times} \backslash\left(\mathbb{A}^{\infty}\right)^{\times} / A_{\wp}^{\times}$under the map nrd.

Observe that the reduced norm induces an isomorphism

$$
\left(B \otimes_{k} \mathbb{A}\right)^{\times a b} \stackrel{\text { nrd }}{\longrightarrow} \mathbb{A}^{\times} .
$$

Hence an automorphic representation of $\left(B \otimes_{k} \mathbb{A}\right)^{\times}$is not one dimensional if and only if it occurs in the cokernel of the pullback homomorphism by the reduced norm

$$
S\left(k^{\times} \backslash \mathbb{A}^{\times}\right) \rightarrow S\left(B^{\times} \backslash\left(B \otimes_{k} \mathbb{A}\right)^{\times}\right) .
$$

Therefore the claim follows from the following result which is stated in [Ja-La, Section 14, p. 470] and is worked out by Gelbart and Jacquet [Ge-Ja, Theorem 8.3, p. 245]: for any automorphic representation $\pi=\otimes_{\wp^{\prime}}^{\prime} \pi_{\wp^{\prime}}$ (here $\wp^{\prime}$ runs over the (possibly the infinity) places of $k$ ) of $\left(B \otimes_{k} \mathbb{A}\right)^{\times}$which is not one dimensional, its "non- $(\wp, \infty)$ " component $\pi^{\wp, \infty}=\otimes_{\wp^{\prime} \neq \wp, \infty}^{\prime} \pi_{\wp^{\prime}}$ is isomorphic to the "non- $(\wp, \infty)$ " component of a cuspidal automorphic representation of $\mathrm{GL}_{2}(\mathbb{A})$. Here we regard $\pi^{\wp, \infty}$ as a representation of $\mathrm{GL}_{2}\left(\mathbb{A}^{\wp, \infty}\right)$ via the isomorphism $\left(B \otimes_{k} \mathbb{A}^{\wp, \infty}\right)^{\times} \cong$ $\mathrm{GL}_{2}\left(\mathbb{A}^{\wp, \infty}\right)$ in Section 5.2.2.

7.2. Recall from Lemma 6.4 that the set of irreducible components of $M_{I_{n} / \kappa(\wp)}^{2}$ is isomorphic to $\mathcal{P}_{n}$ where $n \geq 1$. Let $Q \in \mathcal{P}_{n}$.

Let $M_{I_{n} / \kappa(\wp), Q}^{2, \text { ord }}$ denote the ordinary locus of $M_{I_{n} / \kappa(\wp), Q}^{2}$, that is, the complement of supersingular points of $M_{I_{n} / \kappa(\wp), Q}^{2}$.

In what follows, we consider the two dimensional scheme

$$
M_{I_{n}}^{2, Q}=M_{I_{n} / A_{\wp}}^{2} \backslash \underset{Q^{\prime} \in \mathcal{P}_{n}, Q^{\prime} \neq Q}{\bigcup} M_{I_{n} / \kappa(\wp), Q^{\prime}}^{2} .
$$

We have $M_{I_{n}}^{2, Q} \times_{\operatorname{Spec} A_{\wp}} \operatorname{Spec} \kappa(\wp)=M_{I_{n} / \kappa(\wp), Q}^{2, \text { ord }}$ and $M_{I_{n}}^{2, Q} \times_{\operatorname{Spec} A_{\wp}} \operatorname{Spec} k_{\wp}=$ $M_{I_{n} / k_{\wp}}^{2}$. The localization sequence induces a homomorphism

$$
\partial_{I_{n}, Q}: K_{2}\left(M_{I_{n} / k_{\wp}}^{2}\right) \rightarrow K_{1}\left(M_{I_{n} / \kappa(\wp), Q}^{2, \text { ord }}\right) \cong \mathcal{O}\left(M_{I_{n} / \kappa(\wp), Q}^{2, \text { ord }}\right)^{\times} .
$$

Here the last isomorphism follows from [Ba-Mi-Se, Corollary 4.3 a), p. 95] in view of [Mi, p.27-28].

7.2.1. Take a prime element $\pi \in A_{\wp}$. Let us consider the composite

$$
\begin{aligned}
\widetilde{\mu}_{I_{n}, Q}: & \mathcal{O}\left(M_{I_{n} / k}^{2}\right)^{\times} \hookrightarrow \mathcal{O}\left(M_{I_{n} / k_{\wp}}^{2}\right)^{\times} \\
& \stackrel{\{, \pi\}}{\longrightarrow} K_{2}\left(M_{I_{n} / k_{\wp}}^{2}\right) \stackrel{\partial_{I_{n}, Q}}{\longrightarrow} \mathcal{O}\left(M_{I_{n} / \kappa(\wp), Q}^{2, \text { ord }}\right)^{\times} .
\end{aligned}
$$

Lemma 7.2. For $u \in \mathcal{O}\left(M_{I_{n} / k}^{2}\right)^{\times}$, the function $\widetilde{\mu}_{I_{n}, Q}(u)$, restricted to a connected component of $M_{I_{n} / \kappa(\wp), Q}^{2, \text { ord }}$, has the same order of pole or zero at each supersingular point.

Proof. Using Lemma 6.4(4), we identify the set of supersingular points (resp. connected components) of $M_{I_{n} / \kappa(\wp), Q}^{2}$ with $\Sigma_{I, \wp}\left(\right.$ resp. $\left.S_{I, \wp}\right)$. An element in the image 
of $\gamma_{I}: \mathbb{Z}\left[S_{I, \wp}\right] \rightarrow \mathbb{Z}\left[\Sigma_{I, \wp}\right]$ (defined in Section 7.1) is a constant function on $\Sigma_{I, \wp}$ for each connected component. We show that the image of the composite map

$$
\mathcal{O}\left(M_{I_{n} / k}^{2}\right)^{\times} \stackrel{\widetilde{\mu}_{I_{n}, Q}}{\longrightarrow} \mathcal{O}\left(M_{I_{n} / \kappa(\wp), Q}^{2, \text { ord }}\right)^{\times} \stackrel{\text { div }}{\longrightarrow} \mathbb{Z}\left[\Sigma_{I, \wp}\right]
$$

lies in the image of $\gamma_{I}: \mathbb{Z}\left[S_{I, \wp}\right] \rightarrow \mathbb{Z}\left[\Sigma_{I, \wp}\right]$.

Consider the composite with the quotient map, and extend the scalars:

$$
\mathcal{O}\left(M_{I_{n} / k}^{2}\right)^{\times} \rightarrow \mathbb{C}\left[\Sigma_{I, \wp}\right] / \mathbb{C}\left[S_{I, \wp}\right] .
$$

We prove that this map is zero.

We write the above map as the composite

$$
\mathcal{O}\left(M_{I_{n} / k}^{2}\right)^{\times} \rightarrow \mathcal{O}\left(M_{I_{n} / \kappa(\wp), Q}^{2, \text { ord }}\right)^{\times} \otimes_{\mathbb{Z}} \mathbb{Q} \rightarrow \mathbb{C}\left[\Sigma_{I, \wp}\right] / \mathbb{C}\left[S_{I, \wp}\right]
$$

where the first map is $\widetilde{\mu}_{I_{n}, Q}$ followed by the extension of scalars. By Lemma $4.1(2)$, $M_{I_{n} / k}^{2}$ is a smooth curve over $k$, and it was shown in the proof of Lemma 6.1 that it is connected. Let $k\left(I_{n}\right)$ denote its field of constants. Then it can be checked easily that the first map of (7.1) factors through the quotient $\mathcal{O}\left(M_{I_{n} / k}^{2}\right)^{\times} / k\left(I_{n}\right)^{\times}$.

Let $J^{\prime} \subset J \subset A$ be two non-zero ideals such that $\operatorname{Spec}(A / J)$ contains two or more closed points. Assume $J$ is prime to $\wp$.

The level lowering morphism (restricted over $\operatorname{Spec} A_{\wp}$ ) $M_{J^{\prime} / A_{\wp}}^{2} \rightarrow M_{J / A_{\wp}}^{2}$ is finite flat by Lemma 4.1(3). Considering the moduli problem, we see that $M_{J_{n}^{\prime}}^{2, Q}=$ $M_{J_{n}}^{2, Q} \times_{M_{J / A}^{2}} M_{J^{\prime} / A_{\wp}}^{2}$, so that, as a base change, the level lowering morphism $M_{J_{n}^{\prime}}^{2, Q} \rightarrow M_{J_{n}}^{2, Q}$ is also finite flat. Since $M_{J_{n}^{\prime} / \kappa(\wp), Q}^{2, \text { ord }}$ is canonically isomorphic to the fiber product $M_{J_{n} / \kappa(\wp), Q}^{2 \text {,ord }} \times{ }_{M_{J}^{2}} M_{J^{\prime}}^{2}$, the diagram

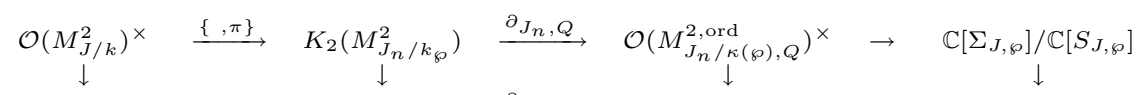

$$
\begin{aligned}
& \mathcal{O}\left(M_{J^{\prime} / k}^{2}\right)^{\times} \stackrel{\{, \pi\}}{\longrightarrow} K_{2}\left(M_{J_{n}^{\prime} / k_{\wp}}^{2}\right) \stackrel{\partial_{J_{n}^{\prime}, Q}}{\longrightarrow} \mathcal{O}\left(M_{J_{n}^{\prime} / \kappa(\wp), Q}^{2 \text { ord }}\right)^{\times} \rightarrow \mathbb{C}\left[\Sigma_{J^{\prime}, \wp}\right] / \mathbb{C}\left[S_{\left.J^{\prime}, \wp\right]}\right]
\end{aligned}
$$

is commutative. We regard the set of ideals $J$ 's as above as a directed set by the inclusion of ideals. Passing to the inductive limit (of row in the diagram above) with respect to $J$ (transition maps are the vertical arrows in the diagram, which is a pullback for each term) we obtain a homomorphism

$$
\begin{aligned}
\mu_{\lim , Q}: & \underset{J, \wp \nmid J}{\lim } \mathcal{O}\left(M_{J_{n} / k}^{2}\right)^{\times} / k\left(J_{n}\right)^{\times} \rightarrow \underset{J, \wp \nmid J}{\lim } \mathcal{O}\left(M_{J_{n} / \kappa(\wp), Q}^{2, \text { ord }}\right)^{\times} \otimes_{\mathbb{Z}} \mathbb{Q} \\
& \rightarrow \mathcal{C}\left(\Sigma_{\lim , \wp}, \mathbb{C}\right) / \mathcal{C}\left(S_{\lim , \wp}, \mathbb{C}\right) .
\end{aligned}
$$

It is $\mathrm{GL}_{2}\left(\mathbb{A}^{\infty, \wp}\right)$-equivariant by Proposition 5.4. Let $\mathbb{K}_{I_{n}} \subset \mathrm{GL}_{2}\left(\mathbb{A}^{\infty}\right)$ be as in Section 4.3.2. Let $\mathbb{K}_{I_{n}}^{\wp}=\mathbb{K}_{I_{n}} \cap \mathrm{GL}_{2}\left(\mathbb{A}^{\infty, \wp}\right)$. It suffices to prove that the image of $\mu_{\lim , Q}$ is zero, since by taking the $\mathbb{K}_{I_{n}}^{\wp}$-invariants, one obtains the claim.

The group $\mathcal{O}\left(M_{J_{n} / k}^{2}\right)^{\times} / k\left(J_{n}\right)^{\times}$is canonically regarded as a subgroup of the abelian group $\mathbb{Z}\left[\operatorname{Cusp}_{J, \wp^{n}}\right]$. Passing to the inductive limit, we have an injective homomorphism

$$
\mathcal{O}\left(M_{J_{n} / k}^{2}\right)^{\times} / k\left(J_{n}\right)^{\times} \rightarrow \mathcal{C}\left(\operatorname{Cusp}_{\lim , \wp^{n}}, \mathbb{Z}\right),
$$

from which we see that Image $\mu_{\lim , Q} \otimes_{\mathbb{Z}} \mathbb{C}$ is isomorphic to a subquotient of the vector space $\mathcal{C}\left(\operatorname{Cusp}_{\lim , \wp^{n}}, \mathbb{C}\right)$.

We prove a lemma, which is a consequence of Lemma 6.3. 
Lemma 7.3. Let $V$ be an irreducible representation of $\mathrm{GL}_{2}\left(\mathbb{A}^{\infty, \wp)}\right)$, which is a subquotient of $\mathcal{C}\left(\mathrm{Cusp}_{\lim , \wp^{n}}, \mathbb{C}\right)$. Let $\pi$ be an irreducible cupidal automorphic representation of $\mathrm{GL}_{2}(\mathbb{A})$. Then $\operatorname{Hom}_{\mathrm{GL}_{2}(\mathbb{A}, \infty)}(V, \pi)=0$.

Proof of Lemma 7.3. Let $V \rightarrow \pi$ be an element of $\operatorname{Hom}_{\mathrm{GL}_{2}\left(\mathbb{A}_{\infty, \wp)}\right.}(V, \pi)$ and consider a cusp form $f$ which belongs to the image of this map. From the definition of a cusp form (see, for example, [Co, p.18, Definition 2.4]), the integral over $N(k) \backslash N(\mathbb{A})$ of $f$ is zero. By the adelic description of $\operatorname{Cusp}_{\lim , \wp^{n}}$ in Lemma 6.3, and using the fact that $N\left(\mathbb{A}^{\infty}\right) N(k) \subset N(\mathbb{A})$ is dense, we see that $f$ is invariant under the action of $N\left(\mathbb{A}^{\infty}\right)$. This implies $f=0$. This proves the claim.

By Lemma 7.1, any irreducible subquotient of the target $\mathcal{C}\left(\Sigma_{\lim , \wp}, \mathbb{C}\right) / \mathcal{C}\left(S_{\lim , \wp}, \mathbb{C}\right)$ of $\mu_{\lim , Q}$ is cuspidal automorphic. Hence $\mu_{\lim , Q} \otimes_{\mathbb{Z}} \mathbb{C}$ is zero. This completes the proof of Lemma 7.2.

Lemma 6.5 together with Lemma 7.2 implies the following corollary :

Corollary 7.4. Let $x \in K_{2}\left(M_{I_{n} / k}^{2}\right)$ be an element which lies in both the image of the symbol map $\mathcal{O}\left(M_{I_{n} / k}^{2}\right)^{\times} \otimes_{\mathbb{Z}} \mathcal{O}\left(M_{I_{n} / k}^{2}\right)^{\times} \rightarrow K_{2}\left(M_{I_{n} / k}^{2}\right)$ and the kernel of the boundary map $K_{2}\left(M_{I_{n} / k}^{2}\right) \rightarrow K_{1}\left(\bar{M}_{I_{n} / k}^{2} \backslash M_{I_{n} / k}^{2}\right) \otimes_{\mathbb{Z}} \mathbb{Q}$. Then for any $Q \in \mathcal{P}_{n}$ (defined in Section 6.2.1), the element $\partial_{I_{n}, Q}(x) \in \mathcal{O}\left(M_{I_{n} / \kappa(\wp), Q}^{2, \text { ord }}\right)^{\times}$is of finite order. Proof. The argument here follows that given in [Sc-Sc, Section 7.3, p. 300].

Write $x=\sum_{i=1}^{N}\left\{u_{1 i}, u_{2 i}\right\}$ with $u_{i 1}, u_{2 i} \in \mathcal{O}\left(M_{I_{n} / k}^{2}\right)^{\times}$for some positive integer $N$. We fix $Q \in \mathcal{P}_{n}$. Then the special fiber of $M_{I_{n}}^{2, Q}$ is a divisor and defines a valuation on the $\operatorname{ring} \mathcal{O}\left(M_{I_{n} / k_{\wp}}^{2}\right)$. In the localization of $\mathcal{O}\left(M_{I_{n} / k_{\wp}}^{2}\right)$ with respect to the valuation above, we may write $u_{1 i}=v_{1 i} \varpi^{a_{1 i}}$ and $u_{2 i}=v_{2 i} \varpi^{a_{2 i}}$ so that the order of $v_{1}$ and of $v_{2}$ are zero with respect to the valuation and $\varpi$ is an element with valuation 1 . Then since $\partial_{I_{n}, Q}(x)=\sum_{i=1}^{N}(-1)^{a_{1 i} a_{2 i}} \frac{v_{1 i}^{a_{2 i}}}{v_{2 i}^{a_{1 i}}}$, we only need to consider the case $N=1, a_{11}=0$ and $a_{21}=1$.

From Lemma 7.2, we see that the restriction of $\partial_{I_{n}, Q}(x)$ to a connected component has the same number of pole of zero at each supersingular point. From the assumption that $x$ lies in the kernel of the boundary map together with Lemma 6.5, it follows that $\partial_{I_{n}, Q}(x)$ has no zero or pole at the cusps. Hence $\partial_{I_{n}, Q}(x)$ on each connected component is a constant function, so that it is of finite order since $\kappa(\wp)$ is finite.

7.3. We present the result of Sections 7.1 and 7.2 in the form to be used in Proposition 9.1. The proof only requires some diagram chasing. We begin with a lemma.

Lemma 7.5. Let $Z$ be a projective smooth curve over a finite field $\mathbb{F}$. Then $G_{1}(Z)$ is torsion.

Proof. We have a localization sequence

$$
\bigoplus_{z} G_{1}(\kappa(z)) \rightarrow G_{1}(Z) \rightarrow G_{1}(\mathbb{F}(Z)) \stackrel{(1)}{\longrightarrow} \bigoplus_{z} G_{0}(\kappa(z))
$$

where $z$ runs over all closed points of $Z$, and $\mathbb{F}(Z)$ is the function field of $Z$. Since $\kappa(z)$ is finite, $G_{1}(\kappa(z))$ is torsion. It is known that $G_{1}(\mathbb{F}(Z))=\mathbb{F}(Z)^{\times}$and the map (1) is given by the order of the function $f \in \mathbb{F}(Z)^{\times}$at each $z$. Since $Z$ is projective, the kernel of the map (1) must be torsion. This proves the claim. 
Proposition 7.6. Let $I \subset A$ be an ideal such that $\operatorname{Spec}(A / I)$ contains two or more closed points. Let $x \in K_{2}\left(M_{I / k}^{2}\right)$ be an element which lies in both the image of the symbol map $\mathcal{O}\left(M_{I / k}^{2}\right)^{\times} \otimes_{\mathbb{Z}} \mathcal{O}\left(M_{I / k}^{2}\right)^{\times} \rightarrow K_{2}\left(M_{I / k}^{2}\right)$ and the kernel of the boundary map $K_{2}\left(M_{I / k}^{2}\right) \rightarrow K_{1}\left(\bar{M}_{I / k}^{2} \backslash M_{I / k}^{2}\right) \otimes_{\mathbb{Z}} \mathbb{Q}$. Let $\wp \subset A$ be a nonzero prime ideal. Then $\partial_{\wp \mathbb{Q}}(x)=0$ where $\partial_{\wp \mathbb{Q}}: K_{2}\left(M_{I / k}^{2}\right)_{\mathbb{Q}} \rightarrow G_{1}\left(M_{I / \kappa(\wp)}^{2}\right)_{\mathbb{Q}}$ is the boundary map.

Proof. Suppose $\wp$ is prime to $I$. Then $\bar{M}_{I / \kappa(\wp)}^{2}$ is a smooth projective curve by Lemma $4.1(2)$ and the proof of Lemma 6.5. Hence $\partial_{\wp}(x)=0$ since the target space is zero by Lemma 7.5.

Suppose $\wp$ divides $I$. Consider the following diagram:

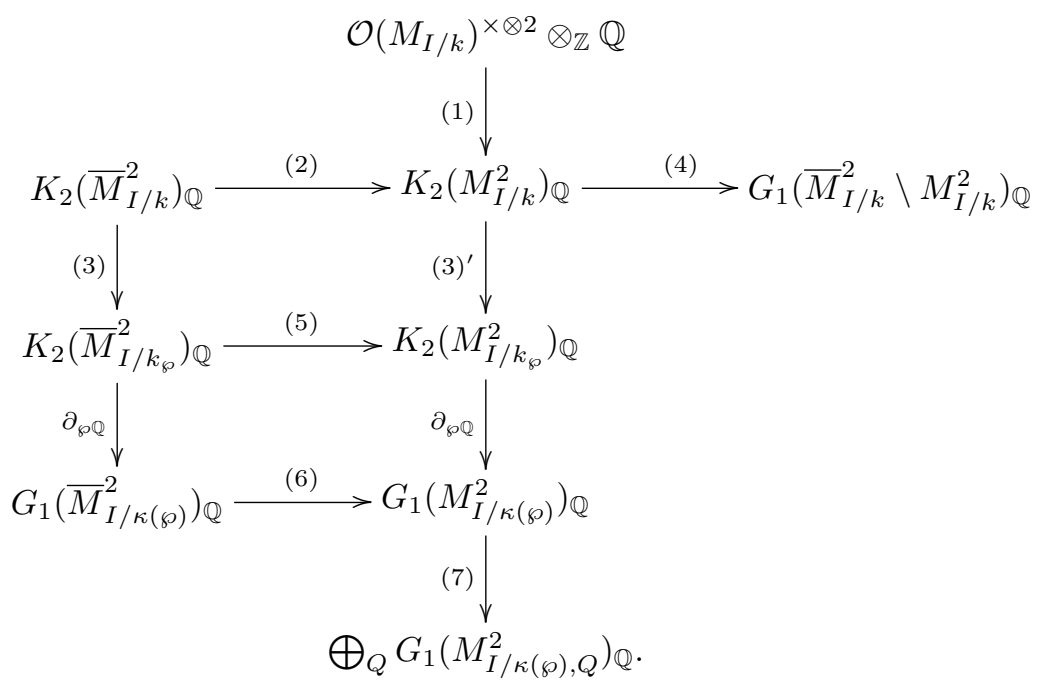

Here the map (1) is the symbol map, the map (2) (5) (6) and (7) are restriction maps (pullback by an open immersion), the map (4) is the boundary map in the localization sequence, and the map (3) and $(3)^{\prime}$ are base change maps. We use $\partial_{\wp}$, by abuse, for the boundary maps. Note that the diagram is commutative.

Define $I^{\prime}$ and $n$ by $I=I^{\prime} \wp^{n}$ where $I^{\prime}$ is prime to $\wp$. Then the composite $(7) \circ \partial_{\wp} \mathbb{Q}$ equals $\bigoplus_{Q} \partial_{I_{n}^{\prime}, Q}$ where $\partial_{I_{n}^{\prime}, Q}$ is as defined above.

The map (6) (resp. (7)) is injective since the kernel is the image of the pushforward from $\bigoplus_{p} G_{1}(p)_{\mathbb{Q}}$ where $p$ runs over the cusps (resp. supersingular points).

Thus to prove the proposition, it suffices to show that the image of $x \in K_{2}\left(\bar{M}_{I / k}^{2}\right)_{\mathbb{Q}}$ by the composite $(7) \partial_{\wp \mathbb{Q}}(3)^{\prime}(2)$ is zero when $(2)(x)$ lies in the image of $(1)$ and the kernel of (4). This is exactly the claim of Corollary 7.4. This finishes the proof.

\section{Regulator}

In this section, we reproduce the results of our other paper ([Ko-Ya $]$ ) in the form to be used in this article. Let us briefly comment on the contents of [Ko-Ya]. In the paper $\left[\mathrm{Ko}-\mathrm{Ya}\right.$ ] we consider the rational $d$-th K-group of the moduli $M_{I / k}^{d}$ over $k$ of Drinfeld modular variety of rank $d$ level $I$. We construct a map called regulator to the space of ( $\mathbb{Q}$-valued) automorphic forms (with certain conditions at infinity) and prove the surjectivity. The case of rank two, in a more precise form, is quoted below as Lemma 8.1. The contents of Sections 7 and 9 (especially Proposition 9.1) may 
be understood as considering the compactified moduli space $\bar{M}_{I}^{2}$ over $A$ instead of $M_{I / k}^{2}$ (which is over $k$ ). The analogous problem, i.e., to construct a surjection from the rational $d$-th $\mathrm{K}$-group of a compactification of the moduli over $A$, remains open for $d \geq 3$.

In Section 8.1, we recall the definitions of the Bruhat-Tits building associated to $\mathrm{PGL}_{2}$ of a non Archimedean local field, and harmonic cochains. In Section 8.2, we recall the definition of the regulator map, which is a homomorphism from the $K_{2}$ of the Drinfeld modular curve to the space of $\mathbb{Z}$-valued harmonic cochains. The details on the regulator map will be used in the proof of Lemmas 9.2 and 9.4.

\subsection{Bruhat-Tits building and harmonic cochains.}

8.1.1. Let $\mathcal{B T}$ denote the Bruhat-Tits building for $\mathrm{PGL}_{2}\left(k_{\infty}\right)$. It is a simplicial complex of dimension one. The set of vertices $\mathcal{B T}_{0}$ (resp. of oriented edges $\mathcal{B T}_{1}$ ) is canonically isomorphic to $\mathrm{GL}_{2}\left(k_{\infty}\right) / k_{\infty}^{\times} \mathrm{GL}_{2}\left(\mathcal{O}_{\infty}\right)$ (resp. $\mathrm{GL}_{2}\left(k_{\infty}\right) / k_{\infty}^{\times} \mathcal{I}$ where $\mathcal{I} \subset \mathrm{GL}_{2}\left(\mathcal{O}_{\infty}\right)$ is the Iwahori subgroup).

For $v \in \mathcal{B T}_{1}$, let $s(v) \in \mathcal{B T}_{0}$ denote the source of the oriented edge $v$. For an abelian group $B$, a $B$-valued harmonic cochain on $\mathcal{B T}$ is an element $f \in$ $\operatorname{Map}\left(\mathcal{B T}_{1}, B\right)$ such that $\sum_{s(v)=e} f(v)=0$ holds for all $v \in \mathcal{B T}_{0}$ and $f\left(e_{v_{1}, v_{2}}\right)=$ $-f\left(e_{v_{2}, v_{1}}\right)$ where $e_{v_{1}, v_{2}}$ means the oriented edge with source $v_{1}$ and target $v_{2}$.

8.1.2. Let $I \subset A$ be a nonzero ideal. Let $\mathcal{B} \mathcal{T}_{I}=\mathrm{GL}_{2}\left(\mathbb{A}^{\infty}\right) / \mathbb{K}_{I} \times \mathcal{B T}=\coprod_{\gamma \in \mathrm{GL}_{2}\left(\mathbb{A}^{\infty}\right) / \mathbb{K}_{I}} \mathcal{B \mathcal { T }}$ (recall $\mathbb{K}_{I}$ from Section 4.3.2). We let $H_{I}$ denote the $\mathbb{Z}$-module of $\mathbb{Z}$-valued harmonic cochains on $\mathcal{B} \mathcal{T}_{I}$ (i.e., an element of $\operatorname{Map}\left(\coprod_{\gamma \in \mathrm{GL}_{2}\left(\mathbb{A}^{\infty}\right) / \mathbb{K}_{I}} \mathcal{B} \mathcal{T}_{1}, \mathbb{Z}\right)$ that is a harmonic cochain when restricted to a component) that are $\mathrm{GL}_{2}(k)$-invariant. We regard $H_{I}$ as a submodule of $\operatorname{Map}\left(\mathrm{GL}_{2}(k) \backslash \mathrm{GL}_{2}(\mathbb{A}) / \mathbb{K}_{I} \mathcal{I}, \mathbb{Z}\right)$ via the canonical isomorphism $\mathcal{B} \mathcal{T}_{1} \cong \mathrm{GL}_{2}\left(k_{\infty}\right) / k_{\infty}^{\times} \mathcal{I}$. We let $H_{0, I} \subset H_{I}$ denote the submodule of elements which have compact support.

We let $H=\lim _{\longrightarrow} H_{I}$ and $H_{0, I}=\lim _{\longrightarrow} H_{0, I}$. We regard them as subsets $H_{0} \subset$

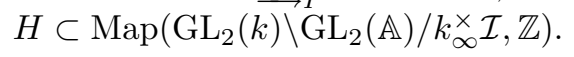

8.1.3. For an abelian group $B$, we let $H_{B}$ and $H_{0, B}$ denote $H \otimes_{\mathbb{Z}} B$ and $H_{0} \otimes_{\mathbb{Z}} B$ respectively. There is an analogue of Petersson inner product $\langle\rangle:, H_{0, \mathbb{Q}} \times H_{\mathbb{Q}} \rightarrow \mathbb{Q}$ defined as $\left\langle f_{1}, f_{2}\right\rangle=\int_{\mathrm{GL}_{2}(k) k_{\infty}^{\times} \backslash \mathrm{GL}_{2}(\mathbb{A})} f_{1}(g) f_{2}(g) d g$, where $d g$ denotes the Haar measure of $\mathrm{GL}_{2}(\mathbb{A})$ with $\operatorname{vol}\left(\prod_{\wp} \mathrm{GL}_{2}\left(A_{\wp}\right)\right)=1$. Since the restriction of $\langle$,$\rangle to$ $H_{0, I, \mathbb{Q}} \times H_{0, I, \mathbb{Q}}$ is non-degenerate, there exists a unique homomorphism $P_{I}: H_{I, \mathbb{Q}} \rightarrow$ $H_{0, I, \mathbb{Q}}$ such that $\left\langle f_{1}, f_{2}\right\rangle=\left\langle f_{1}, P_{I} f_{2}\right\rangle$ for all $f_{1} \in H_{0, I, \mathbb{Q}}, f_{2} \in H_{I, \mathbb{Q}}$. Passing to the inductive limit we obtain a $\mathrm{GL}_{2}(\mathbb{A})$-equivariant homomorphism $P: H_{\mathbb{Q}} \rightarrow H_{0, \mathbb{Q}}$.

8.2. Regulator. Let $\mathcal{B T}$ denote the Bruhat-Tits building for $\mathrm{PGL}_{2}\left(k_{\infty}\right)$. Let $I \subset A$ be a non-zero ideal such that $\operatorname{Spec}(A / I)$ contains two or more closed points. In [Ko-Ya, p.22, Section 7.3], the authors defined the regulator map

$$
\operatorname{reg}_{I}: K_{2}\left(M_{I / k}^{2}\right) \rightarrow H_{I} .
$$

Let us recall the construction.

Let $\mathfrak{X}=\mathfrak{X}_{2}$ be the Drinfeld upper half plane (the Drinfeld symmetric space of dimension one) over $k_{\infty}$. The rigid analytic uniformization of $M_{I}^{2}$ (see Section 4.3.4 (4.2)) gives a canonical morphism

$$
\coprod_{\gamma \in \mathrm{GL}_{2}\left(\mathbb{A}^{\infty}\right) / \mathbb{K}_{I}} \mathfrak{X} \rightarrow\left(M_{I / k_{\infty}}^{2}\right)^{\text {an }}
$$


of rigid analytic spaces. For $\gamma \in \mathrm{GL}_{2}\left(\mathbb{A}^{\infty}\right)$, let $v_{\gamma}: \mathfrak{X} \rightarrow\left(M_{I / k_{\infty}}^{2}\right)^{\text {an }}$ denote its $\gamma \mathbb{K}_{I}$-component.

There is the specialization map: sp : $\mathfrak{X} \rightarrow \mathcal{B T}$ or the reduction map [DeS, Part II, 6.1, p. 157] (we note that this specialization map is different from the map sp introduced in Section 4.3). Let $e$ be an oriented edge (including the endpoints) in the Bruhat-Tits building $\mathcal{B T}$. Then $\mathrm{sp}^{-1}(e)$ is isomorphic to $\operatorname{Spm} B_{e}$ for some affinoid algebra $B_{e}$. Let $Y_{e}$ denote the special fiber of the formal model of $\operatorname{Spm} B_{e}$. It is known that $Y_{e}$ has two irreducible components $C_{s}$ and $C_{t}$ (corresponding to the source and the target of the edge $e$ ) intersecting at a $\kappa(\wp)$-rational point. Each irreducible component is isomporphic to an open subset of the projective line over $\kappa(\wp)$. Let $v_{\gamma, e}: \operatorname{Spm} B_{e} \rightarrow\left(M_{I / k_{\infty}}^{2}\right)$ an denote the restriction of $v_{\gamma}$ to $\operatorname{sp}^{-1}(e)$. Associated to $v_{\gamma, e}$, by the functoriality of rigid analytic functions for a morphisms of rigid analytic spaces, there is a canonical ring homomorphism $\mathcal{O}\left(M_{I / k_{\infty}}^{2}\right) \rightarrow B_{e}$. Consider

$$
K_{2}\left(M_{I / k}^{2}\right) \rightarrow \prod_{\gamma, e} K_{2}\left(B_{e}\right) \stackrel{\partial}{\rightarrow} \prod_{\gamma, e} G_{1}\left(Y_{e}\right)
$$

where the first map is the pullback map induced by the canonical ring homomorphism above, the second is the boundary map in the localization sequence of the pair $\left(\operatorname{Spec} B_{e}\right.$, Spec $\left.B_{e} /\left(\pi_{\infty}\right)\right)\left(\pi_{\infty}\right.$ is a uniformizer of $\left.k_{\infty}\right)$. Let $p$ denote the intersection of the two irreducible components of $Y_{e}$. We have $G_{1}\left(Y_{e}\right) \rightarrow G_{1}\left(C_{s} \backslash\{p\}\right) \rightarrow G_{0}(p)$ where the first map is the restriction map and the second map is the boundary map in the localization sequence.

Let $x \in K_{2}\left(M_{I / k}^{2}\right)$. For $\gamma \in \mathrm{GL}_{2}\left(\mathbb{A}^{\infty}\right) / \mathbb{K}_{I}$ and $v \in \mathcal{B} \mathcal{T}_{1}$. We define $\operatorname{reg}_{I}(x)((\gamma, v))$ to be the image of $x$ under the composition of the maps above to $G_{0}(p) \cong \mathbb{Z}$.

8.3. Let us state the results from our other paper $[\mathrm{Ko}-\mathrm{Ya}]$.

For each non-zero ideal $I \subset A$ such that $\operatorname{Spec}(A / I)$ contains two or more closed points, let $W_{I}=\operatorname{Image}\left[\mathcal{O}\left(M_{I / k}^{2}\right)^{\times} \otimes \mathcal{O}\left(M_{I / k}^{2}\right)^{\times} \rightarrow K_{2}\left(M_{I / k}^{2}\right)\right]$. We put $W=$ $\lim _{I} W_{I}$.

Lemma 8.1. Let the notations be as above. The composite

$$
\operatorname{reg}_{\mathbb{Q}}^{\prime}: W_{\mathbb{Q}} \stackrel{\text { reg }}{\longrightarrow} H_{\mathbb{Q}} \stackrel{P}{\longrightarrow} H_{0, \mathbb{Q}}
$$

is surjective.

Proof. It follows from [Ko-Ya, p.23, Theorem 9.1].

\section{ON THE COMPACTIFICATION}

The aim of this section is to prove Proposition 9.1. This is the only result to be used in other sections, and will be used only in Section 11.1.

Lemma 8.1 says that there are certain nontrivial elements in the $K_{2}$ of the (open) Drinfeld modular curves. In this section, we construct elements in the $K_{2}$ of the compactifcation of the Drinfeld modular curves, starting from those elements, without modifying the property (namely the surjectivity of certain homomorphism) at the prime $\infty$.

The details of the procedure in the case of the moduli of elliptic curves are given in [De-Wi]. The key ingredient there is the Drinfeld-Manin theorem on the finiteness of the cuspidal divisor group of Drinfeld modular curves. Using its Drinfeld modular 
analogue proved by Gekeler ([Gek1]), we give the details for the Drinfeld modular case in this section.

In Section 9.1, we give the statement of the main result of this section. The result of Section 9.2 has a rather direct analogue in the elliptic modular case. Lemma 9.4 of Section 9.3 requires some computation regarding K-groups. The procedures in the proof of Lemma 9.4 would be much clearer if we had K-theory for rigid analytic spaces. As we do not, we do some diagram chasing.

9.1. For each prime $\wp$ of $k$, the homomorphism

$$
K_{2}\left(\bar{M}_{I / k}^{2}\right) \rightarrow K_{2}\left(\bar{M}_{I / k_{\wp}}^{2}\right) \stackrel{c_{2,2}}{\longrightarrow} H_{\text {ét }}^{2}\left(\bar{M}_{I / k_{\wp}}^{2}, \mathbb{Q}_{\ell}(2)\right)
$$

gives rise to the homomorphism $K_{2}\left(\bar{M}_{\lim , k}^{2}\right) \rightarrow H_{\text {ét }}^{2}\left(\bar{M}_{\lim , k_{\wp}}^{2}, \mathbb{Q}_{\ell}(2)\right)$ which we denote by $c_{2,2, \wp}$.

For each non-zero ideal $I \subset A$ such that $\operatorname{Spec}(A / I)$ contains two or more closed points, let $W_{I}=$ Image $\left[\mathcal{O}\left(M_{I / k}^{2}\right)^{\times} \otimes \mathcal{O}\left(M_{I / k}^{2}\right)^{\times} \rightarrow K_{2}\left(M_{I / k}^{2}\right)\right]$. We let $W_{0, I}$ denote the inverse image of $W_{I}$ under the homomorphism $K_{2}\left(\bar{M}_{I / k}^{2}\right) \rightarrow K_{2}\left(M_{I / k}^{2}\right)$. We put $W=\lim _{\longrightarrow} W_{I}$ and $W_{0}=\lim _{\longrightarrow} W_{0, I}$.

Proposition 9.1. Let $\wp$ be a prime of $k$. The composite

$$
W_{0} \otimes_{\mathbb{Z}} \mathbb{Q}_{\ell} \rightarrow K_{2}\left(\bar{M}_{\lim , k}^{2}\right) \otimes_{\mathbb{Z}} \mathbb{Q}_{\ell} \stackrel{c_{2,2, \wp}}{\longrightarrow} H_{e ́ t}^{2}\left(\bar{M}_{\lim , k_{\wp}}^{2}, \mathbb{Q}_{\ell}(2)\right)
$$

is surjective if $\wp=\infty$. If $\wp \neq \infty$, then it equals zero.

Proof. The case $\wp \neq \infty$ follows from Proposition 7.6 using Proposition 3.1. The rest of this section is devoted to the proof of the case $\wp=\infty$.

9.2. Pulling back by the determinant morphism (defined in Section 4.4), we may regard elements of $\mathcal{O}\left(M_{I / k}^{1}\right)$ as elements of $\mathcal{O}\left(M_{I / k}^{2}\right)$.

Lemma 9.2. Let $u_{1} \in \mathcal{O}\left(M_{I / k}^{1}\right)^{\times}$and $u_{2} \in \mathcal{O}\left(M_{I / k}^{2}\right)^{\times}$. Then $P \circ \operatorname{reg}_{I}\left(\left\{u_{1}, u_{2}\right\}\right)$ equals zero.

Proof. From the definition of $\operatorname{reg}_{I}$ (Section 8.2), we have

$$
\operatorname{reg}_{I}\left(\left\{u_{1}, u_{2}\right\}\right)(e)=\operatorname{det}\left(\begin{array}{cc}
\operatorname{ord}_{C_{t(e)}} u_{1} & \operatorname{ord}_{C_{t(e)}} u_{2} \\
\operatorname{ord}_{C_{s(e)}} u_{1} & \operatorname{ord}_{C_{s(e)}} u_{2}
\end{array}\right)
$$

where $s(e)$ (resp. $t(e))$ is the source (resp. the target) of the oriented edge $e$, and $C_{v}$ is the component corresponding to the vertex $v$ (see Section 8.2). As $u_{1} \in \mathcal{O}\left(M_{I / k}^{1}\right)^{\times}$, we see that there exists a function $h$ on the set of vertices such that $\operatorname{reg}_{I}\left(\left\{u_{1}, u_{2}\right\}\right)(e)=h(t(e))-h(s(e))$, i.e., it is a 1-coboundary.

For a harmonic cochain $f \in H_{0, I}$ with compact support, we have

$$
\left\langle f, \operatorname{reg}_{I}\left(\left\{u_{1}, u_{2}\right\}\right)\right\rangle=\sum_{e} f(e)[h(t(e))-h(s(e))]=\sum_{v} \sum_{t(e)=v} f(e) h(t(e))-\sum_{v} \sum_{s(e)=v} f(e) h(s(e)) .
$$

Both quantities on the right hand side are zero by the harmonicity of $f$. This finishes the proof.

Let the notations be as above. 
Lemma 9.3. The composite

$$
W_{0, \mathbb{Q}} \rightarrow W_{\mathbb{Q}} \rightarrow K_{2}\left(M_{\lim , k}^{2}\right)_{\mathbb{Q}} \stackrel{\mathrm{reg}}{\longrightarrow} H_{\mathbb{Q}} \stackrel{P}{\rightarrow} H_{0, \mathbb{Q}}
$$

is surjective.

Proof. We apply the method of [De-Wi, Lemma 5.2, p. 265] in the case of elliptic modular curves (called Bloch's method there) to our Drinfeld modular context.

Let $h_{0} \in H_{0, \mathbb{Q}}$. By Lemma 8.1, one can find an element $\kappa$ in the image of the map $W_{I, \mathbb{Q}} \rightarrow K_{2}\left(M_{I / k}^{2}\right){ }_{\mathbb{Q}}$ which maps to $h_{0}$ via $P \circ \operatorname{reg}_{I}$ for some $I$. We show that there exists an element in $W_{0, I, \mathbb{Q}} \subset K_{2}\left(\bar{M}_{I / k}^{2}\right)$ which maps to $\kappa$ via the restriction map $K_{2}\left(\bar{M}_{I / k}^{2}\right) \rightarrow K_{2}\left(M_{I / k}^{2}\right)$. This implies the statement of the lemma.

We note that every cusp of $\bar{M}_{I / k}^{2}$ is an $M_{I / k^{-}}^{1}$ valued point (see [Le, p.82, Proposition 3.5]). We also recall that $M_{I / k}^{1}$ is the spectrum of a field (see Lemma 4.2). Consider the exact sequence

$$
K_{2}\left(\bar{M}_{I / k}^{2}\right) \stackrel{\rho}{\rightarrow} K_{2}\left(M_{I / k}^{2}\right) \stackrel{\mu}{\rightarrow} K_{1}\left(\bar{M}_{I / k}^{2} \backslash M_{I / k}^{2}\right) \stackrel{\nu}{\rightarrow} K_{1}\left(\bar{M}_{I / k}^{2}\right) .
$$

The last term $K_{1}\left(\bar{M}_{I / k}^{2}\right)$ tensored with $\mathbb{Q}$ contains a direct summand isomorphic to $\left(\mathcal{O}\left(\bar{M}_{I / k}^{2}\right)^{\times}\right)_{\mathbb{Q}}$ (see, for example, [B11, p.267-269]). Using Lemma 6.1(2), we see that $\left(\mathcal{O}\left(\bar{M}_{I / k}^{2}\right)^{\times}\right)_{\mathbb{Q}} \cong\left(\mathcal{O}\left(M_{I / k}^{1}\right)^{\times}\right)_{\mathbb{Q}}$. An element $\left(f_{c}\right)_{c} \in \bigoplus_{c} \mathcal{O}\left(M_{I / k}^{1}\right)^{\times}=$ $K_{1}\left(\bar{M}_{I / k}^{2} \backslash M_{I / k}^{2}\right)$ is sent via $\nu$ to (the class of) $\prod_{c} f_{c}$ in the summand. Here $c$ runs over the set of cusps in each case. Let $\kappa \in K_{2}\left(M_{I / k}^{2}\right)$, and let $\left(f_{c}\right)_{c}$ be its image in $K_{1}\left(\bar{M}_{I / k}^{2} \backslash M_{I / k}^{2}\right)$. By the exactness, we have $\prod_{c} f_{c}=1$.

The Drinfeld modular analogue of the theorem of Drinfeld and Manin is proved by Gekeler ([Gek1, 1.2 Theorem, p. 359]). (Gekeler proves that the cuspidal divisor class group of the Drinfeld modular curve associated to a congruence subgroup $\Gamma$, denoted $\bar{M}_{\Gamma}$ there, which is defined over some finite extension of the base field, is finite. To apply it to our case, use that there exists a finite field extension $L \supset k$ such that there exists a finite flat map $h: \bar{M}_{I / L}^{2} \rightarrow \bar{M}_{\Gamma}$ which sends cusps to cusps. Then as $h_{*} h^{*}=\operatorname{deg} h$ on the G-group we obtain $N$ below.) We can take an integer $N$ large enough so that $N$ annihilates the cuspidal subgroup. Let us fix a cusp, say $c_{0}$, and consider an element $g_{c} \in \mathcal{O}\left(M_{I / k}^{2}\right)^{\times}$whose divisor is $N\left([c]-\left[c_{0}\right]\right)$ for each cusp $c$. We put $u=\sum_{c} g_{c} \otimes f_{c} \in \mathcal{O}\left(M_{I / k}^{2}\right)^{\times} \otimes \mathcal{O}\left(M_{I / k}^{1}\right)^{\times}$. Then the image of $u$ under the $\operatorname{map} \mathcal{O}\left(M_{I / k}^{2}\right)^{\times} \otimes \mathcal{O}\left(M_{I / k}^{1}\right)^{\times} \rightarrow K_{2}\left(M_{I / k}^{2}\right) \rightarrow K_{1}\left(\bar{M}_{I / k}^{2} \backslash M_{I / k}^{2}\right)$ equals $N \mu(\kappa)$. Thus for each $\kappa \in K_{2}\left(M_{I / k}^{2}\right)$, one can find an element $\bar{\kappa}$ in Image $\left[K_{2}\left(\bar{M}_{I / k}^{2}\right) \rightarrow K_{2}\left(M_{I / k}^{2}\right)\right]$ such that $\bar{\kappa}-N \kappa$ belongs to Image $\left[\mathcal{O}\left(M_{I / k}^{2}\right)^{\times} \otimes \mathcal{O}\left(M_{I / k}^{1}\right)^{\times} \rightarrow K_{2}\left(\bar{M}_{I / k}^{2}\right)\right]$. Using Lemma 9.2, we obtain the claim.

9.3.

Lemma 9.4. The kernel of $\mathrm{reg}_{I}$ contains the kernel of the composite

$$
K_{2}\left(M_{I / k}^{2}\right) \rightarrow K_{2}\left(M_{I / k_{\infty}}^{2}\right) \stackrel{c_{2,2}}{\longrightarrow} H_{e t}^{2}\left(M_{I / k_{\infty}}^{2}, \mathbb{Q}_{\ell}(2)\right) .
$$


Proof. By the definition of reg $_{I}$ the kernel of reg ${ }_{I}$ contains the kernel of the composite

$$
K_{2}\left(M_{I / k}^{2}\right) \rightarrow \prod_{\gamma, e} K_{2}\left(B_{e}\right) \stackrel{\partial}{\rightarrow} \prod_{\gamma, e} G_{1}\left(Y_{e}\right) \rightarrow \prod_{\gamma, e} G_{1}\left(Y_{e}\right) / G_{1}\left(Y_{e}\right)_{\mathrm{tors}}
$$

where the first two maps are as in (8.1) and the third is the canonical surjection. Let $K, L_{e}$ denote the total quotient ring of $\mathcal{O}\left(M_{I / k_{\infty}}^{2}\right), \mathcal{O}\left(Y_{e}\right)$, respectively. Let $\operatorname{Frac}\left(B_{e}\right)$ denote the field of fractions of $B_{e}$. Consider the following commutative diagram:

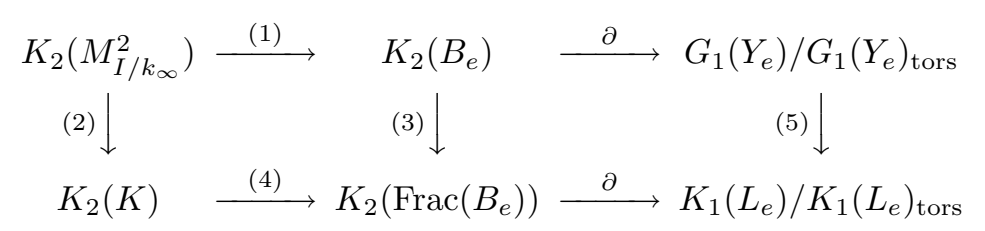

where the map (1) is the map induced by $v_{\gamma, e}$; the maps (2) (3) (4) are those induced by the canonical homomorphisms of rings; the top $\partial$ is as in (8.1); the bottom $\partial$ is the norm map of Milnor K-theory with respect to the valuation given by the uniformizer of $k_{\infty}$; the vertical map (5) is induced by the pullback map to the generic points of the irreducible components.

We note that the homomorphism (5) is injective since $Y_{e}$ is a normal crossing curve over a finite field. Hence the kernel of $\operatorname{reg}_{I}$ contains the kernel of the composite

$$
K_{2}\left(M_{I / k}^{2}\right) \rightarrow K_{2}(K) \rightarrow \prod_{\gamma, e} K_{2}\left(\operatorname{Frac}\left(B_{e}\right)\right) \stackrel{\partial}{\rightarrow} \prod_{\gamma, e} K_{1}\left(L_{e}\right) / K_{1}\left(L_{e}\right)_{\mathrm{tors}}
$$

For an abelian group $M$, let $M^{\wedge}=\left(\lim _{n} M / l^{n}\right) \otimes_{\mathbb{Z}} \mathbb{Q}$. The following diagram is commutative:

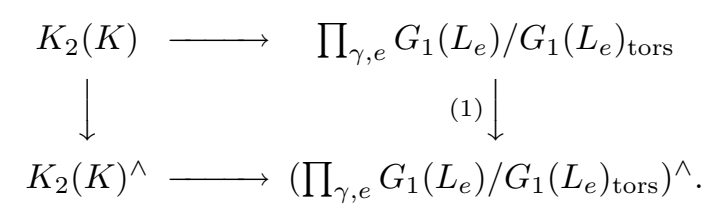

Here the vertical maps are the canonical maps; the map at the top is the map in the diagram above; the map at the bottom is the map induced by the map at the top. Since $L_{e}$ is the product of two copies of the field of rational functions of one variable over a finite field, $G_{1}\left(L_{e}\right) / G_{1}\left(L_{e}\right)_{\text {tors }}$ is a free abelian group. Hence the map (1) is injective. Therefore, the kernel of reg $_{I}$ contains the kernel of the composite

$$
K_{2}\left(M_{I / k}^{2}\right) \rightarrow K_{2}(K) \rightarrow K_{2}(K)^{\wedge} .
$$

We note that $K$ is the product of a finite number of fields. By the theorem of Merkurjev and Suslin ([Me-Su, (11.5), Theorem, p. 328]), the symbol map $K_{2}(K) \rightarrow H_{\text {êt }}^{2}\left(\operatorname{Spec} K, \mathbb{Q}_{\ell}(2)\right)$ gives an isomorphism $K_{2}(K)^{\wedge} \cong H_{\text {êt }}^{2}\left(\operatorname{Spec} K, \mathbb{Q}_{\ell}(2)\right)$. The following diagram, where the maps (1) and (3) are those induced by the canonical inclusions of rings, the map (2) is the canonical map, and the map (4) is the 
pullback, is commutative:

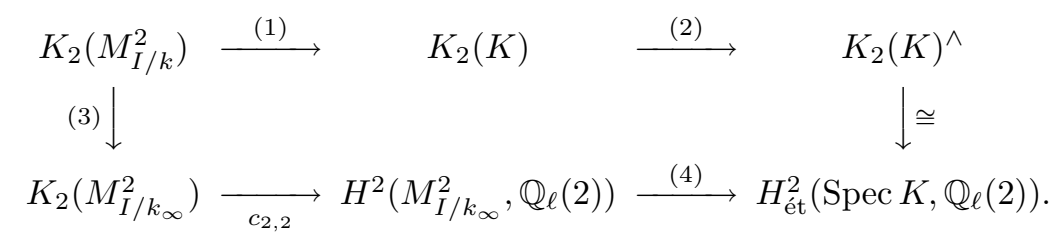

Hence the kernel of (9.2) contains the kernel of the composite map $K_{2}\left(M_{I / k}^{2}\right) \rightarrow$ $K_{2}\left(M_{I / k_{\infty}}^{2}\right) \rightarrow H^{2}\left(M_{I / k_{\infty}}^{2}, \mathbb{Q}_{\ell}(2)\right)$. This completes the proof of Lemma 9.4.

\subsection{Proof of Proposition 9.1.}

Proof of Proposition 9.1. Let $\bar{W}_{0}$ denote the image of

$$
W_{0, \mathbb{Q}_{\ell}} \rightarrow K_{2}\left(\bar{M}_{\mathrm{lim}, k}^{2}\right)_{\mathbb{Q}_{\ell}} \stackrel{c_{2,2, \infty}}{\longrightarrow} H_{\text {ét }}^{2}\left(\bar{M}_{\mathrm{lim}, k_{\infty}}^{2}, \mathbb{Q}_{\ell}(2)\right)
$$

Using Lemma 9.4 and 9.3 , we see that $H_{0, \mathbb{Q}_{e}}$ is a quotient of $\bar{W}_{0}$.

We have ([vdP-Re, Theorem 2.13, p.199]) a canonical isomorphism $H_{0, \mathbb{Q}_{\ell}} \cong$ $\operatorname{Hom}_{\mathrm{GL}_{2}\left(k_{\infty}\right)}\left(\mathrm{St}, \mathcal{A}_{\text {cusp }}\right)$ of $\mathrm{GL}_{2}\left(\mathbb{A}^{\infty}\right)$-modules, where $\mathcal{A}_{\text {cusp }}$ denotes the space of $\mathbb{Q}_{\ell^{-}}$ valued cusp forms on $\mathrm{GL}_{2}(k) \backslash \mathrm{GL}_{2}(\mathbb{A})$, and St denotes the Steinberg representation of $\mathrm{GL}_{2}\left(k_{\infty}\right)$. Let $V_{\ell}$ be a two dimensional $\mathbb{Q}_{\ell}$-representation of $\operatorname{Gal}\left(\bar{k}_{\infty} / k_{\infty}\right)$ which admits a non-split exact sequence

$$
0 \rightarrow \mathbb{Q}_{\ell} \rightarrow V_{\ell} \rightarrow \mathbb{Q}_{\ell}(-1) \rightarrow 0
$$

Such $V_{\ell}$ is unique up to an isomorphism. By the fundamental theorem in [Dr1, $\S 11$, p. 591], we have an isomorphism

$$
\begin{aligned}
H_{\text {ét }}^{2}\left(\bar{M}_{\lim , k_{\infty}}^{2}, \mathbb{Q}_{\ell}(2)\right) & \cong \lim _{\lim ^{1}} H^{1}\left(k_{\infty}, H_{\text {ét }}^{1}\left(\bar{M}_{I / \bar{k}_{\infty}}^{2}, \mathbb{Q}_{\ell}(2)\right)\right) \\
& \left.\cong V_{\ell}\right) \mathbb{Q}_{\ell} \operatorname{Hom}_{\mathrm{GL}_{2}\left(k_{\infty}\right)}\left(\mathrm{St}, \mathcal{A}_{\text {cusp }}\right)
\end{aligned}
$$

of $\mathrm{GL}_{2}\left(\mathbb{A}^{\infty}\right)$-modules. Since $H^{1}\left(k_{\infty}, V_{\ell}\right)$ is one-dimensional, $H_{\text {ét }}^{2}\left(\bar{M}_{\lim , k_{\infty}}^{2}, \mathbb{Q}_{\ell}(2)\right)$ is isomorphic to $H_{0, \mathbb{Q}_{\ell}}$ as a $\mathrm{GL}_{2}\left(\mathbb{A}^{\infty}\right)$-module. On the other hand, $H_{0, \mathbb{Q}_{\ell}}$ is a quotient of the $\mathrm{GL}_{2}\left(\mathbb{A}^{\infty}\right)$-submodule $\bar{W}_{0}$ of $H_{\text {ét }}^{2}\left(\bar{M}_{\lim , k_{\infty}}^{2}, \mathbb{Q}_{\ell}(2)\right)$. Using the multiplicity one theorem, we conclude that the module $\bar{W}_{0}$ equals $H_{\text {ét }}^{2}\left(\bar{M}_{\lim , k_{\infty}}^{2}, \mathbb{Q}_{\ell}(2)\right)$, since $H_{0, \mathbb{Q}_{\ell}}$ is an admissible $\mathrm{GL}_{2}\left(\mathbb{A}^{\infty}\right)$-module. This completes the proof of Proposition 9.1.

\section{MOdular PARAMETRIZATION}

The aim of this section is to recall the modular parametrization of an elliptic curve with a split multiplicative reduction. The reader is referred to [Ge-Re] for the details including some historical account. Let $E$ be an elliptic curve over a global field $k$ of positive characteristic, and suppose that it has split multiplicative reduction at a prime $\infty$. Then a modular parametrization is a non-constant morphism from a Drinfeld modular curve (whose prime at infinity is the prime $\infty$ ) to the ellitpic curve. For (the conditions on) the existence and the construction, the reader is referred to Section 8 of [Ge-Re].

In Section 9, we have considered elements in the rational $K_{2}$ of the compactified Drinfeld modular curves (namely, the image of the first map in Proposition 9.1). We use the pushforward map by the modular parametrization to obtain elements in the rational $K_{2}$ of an elliptic curve over $k$. This will be done in Section 11.1. 
Lemma 10.1 describes the map which is induced by the parametrization on the étale cohomology group of our interest. The surjectivity in question on the cohomology reduces to the surjectivity of the morphism of the Jacobians, which is immediate since the morphism is non-constant and the target is an elliptic curve.

10.1. Let $E$ be an elliptic curve over a global field $k$ of positive characteristic, and let $C$ be the projective smooth curve over a finite field whose function field is $k$. We fix a prime $\infty$ of $k$ at which $E$ has split multiplicative reduction. Let $A=H^{0}\left(C \backslash\{\infty\}, \mathcal{O}_{C}\right)$. We use the notations in Section 4 and Section 5 . We also fix a separable closure $\bar{k}_{\infty}$ of $k_{\infty}$.

Let $I \subsetneq A$ be a non-zero ideal such that $\operatorname{Spec}(A / I)$ contains two or more closed points. For an $A$-algebra $L$, we let $M_{I / L}^{d}=M_{I}^{d} \times_{\operatorname{Spec} A} \operatorname{Spec} L$ and $\bar{M}_{I / L}^{2}=$ $\bar{M}_{I}^{2} \times_{\operatorname{Spec} A} \operatorname{Spec} L$. For a $k$-algebra $L^{\prime}$, we let $E_{L^{\prime}}=E \times_{\operatorname{Spec} k} \operatorname{Spec} L^{\prime}$.

Recall that $\bar{M}_{I}^{2}$ denotes the compactification of the $A$-scheme $M_{I}^{2}$ as constructed by Drinfeld (see the proof of 6.1 where it first appeared). For a field $L \supset k$, let us denote by $M_{\text {lim }, L}^{2}\left(\operatorname{resp} . \bar{M}_{\lim , L}^{2}\right)$ the projective limit $\varliminf_{I}\left(M_{I / L}^{2}\right)\left(\operatorname{resp} \cdot \varliminf_{I}\left(\bar{M}_{I / L}^{2}\right)\right)$ where $I$ runs over the ideals such that $\operatorname{Spec}(A / I)$ contains two or more closed points. These projective limits exist in the category of schemes over $\operatorname{Spec} L$ since all the transition maps are finite.

Let $I_{E}$ denote the finite part of the conductor, regarded also as an ideal of $A$. We use the non-constant morphism $\mathrm{pr}_{E}: J_{0}\left(I_{E}\right) \rightarrow E$ of [Ge-Re, 8.3.10, p.82] (see loc. cit. for the notation $J_{0}\left(I_{E}\right)$ and $M_{0}^{2}\left(I_{E}\right)$ where they use $\mathfrak{n}$ for $\left.I_{E}\right)$. We use it in the form $\varphi_{E}: \bar{M}_{I_{E} / k}^{2} \rightarrow \bar{M}_{0}^{2}\left(I_{E}\right) \rightarrow J_{0}\left(I_{E}\right) \rightarrow E$ where the first map is the canonical quotient map, the second map is the canonical inclusion into the Jacobian, and the third map is $\operatorname{pr}_{E}$ above. For $J \subset I_{E}$, let $\varphi_{E, J}$ denote the composite $\bar{M}_{J / k}^{2} \rightarrow \bar{M}_{I / k}^{2} \rightarrow E$. For a field $L \supset k$, we let $\varphi_{E, J, L}: \bar{M}_{J / L}^{2} \rightarrow \bar{M}_{I / L}^{2} \rightarrow E_{L}$ denote the base change of $\varphi_{E, J}$ to $L$.

We fix a prime number $\ell$ different from $p$. For each prime $\wp$ of $k$, we put

$$
\begin{aligned}
& H_{\text {ét }}^{2}\left(M_{\text {lim }, k_{\wp}}^{2}, \mathbb{Q}_{\ell}(2)\right)=\lim _{\longrightarrow} H_{\text {ét }}^{2}\left(M_{I / k_{\wp}}^{2}, \mathbb{Q}_{\ell}(2)\right), \\
& H_{\text {ét }}^{2}\left(\bar{M}_{\lim , k_{\wp}}^{2}, \mathbb{Q}_{\ell}(2)\right)=\lim _{\longrightarrow} H_{\text {ét }}^{2}\left(\bar{M}_{I / k_{\wp}}^{2}, \mathbb{Q}_{\ell}(2)\right) .
\end{aligned}
$$

where the transition map for the first line is the pullback map $\varphi_{J, J^{\prime}}^{*}: H_{\text {ét }}^{2}\left(M_{J / k_{\wp}}^{2}, \mathbb{Q}_{\ell}(2)\right) \rightarrow$ $H_{\text {ét }}^{2}\left(M_{J^{\prime} / k_{\wp}}^{2}, \mathbb{Q}_{\ell}(2)\right)$ where $\varphi_{J, J^{\prime}}: M_{J^{\prime} / k_{\wp}}^{2} \rightarrow M_{J / k_{\wp}}^{2}$ for $J \subset J^{\prime}$ is the canonical level lowering morphism. The transition map for the second line is defined in a similar manner.

Consider the homomorphisms

$$
\frac{1}{\operatorname{deg}\left(\varphi_{E, J, k_{\wp}}\right)} \cdot \varphi_{E, J, k_{\wp *}}: H_{\text {ét }}^{2}\left(\bar{M}_{J / k_{\wp}}^{2}, \mathbb{Q}_{\ell}(2)\right) \rightarrow H_{\text {ét }}^{2}\left(E_{k_{\wp}}, \mathbb{Q}_{\ell}(2)\right)
$$

where $J \subset I_{E}$ is an ideal such that $\operatorname{Spec}(A / J)$ contains two or more closed points. Let $J^{\prime} \subset J \subset I_{E}$ be two ideals with the same condition. Then since $\varphi_{J, J^{\prime} *} \varphi_{J, J^{\prime}}^{*}=\operatorname{deg} \varphi_{J, J^{\prime}}$, we obtain a homomorphism $\varphi_{*, \wp, \text { ét }}: H_{\text {ét }}^{2}\left(\bar{M}_{\lim , k_{\wp}}^{2}, \mathbb{Q}_{\ell}(2)\right) \rightarrow$ $H_{\text {ét }}^{2}\left(E_{k_{\wp}}, \mathbb{Q}_{\ell}(2)\right)$ as the limit of the homomorphisms $\frac{1}{\operatorname{deg}\left(\varphi_{E, J, k_{\wp}}\right)} \cdot \varphi_{E, J, k_{\wp} *} \cdot$

Lemma 10.1. The homomorphism $\varphi_{*, \wp, \text { ét }}$ is surjective. 
Proof. Let us consider the morphism $\varphi_{E, I}$ for a sufficiently small ideal $I$. Take a separable closure $\bar{k}_{\wp}$ of $k_{\wp}$. We have a commutative diagram

$$
\begin{gathered}
H_{\text {êt }}^{2}\left(\bar{M}_{I / k_{\wp}}^{2}, \mathbb{Q}_{\ell}(2)\right) \longrightarrow H^{1}\left(k_{\wp}, H_{\text {êt }}^{1}\left(\bar{M}_{I / \bar{k}_{\wp}}^{2}, \mathbb{Q}_{\ell}(2)\right)\right) \\
\varphi_{E, I, k_{\wp}} \downarrow \\
H_{\text {êt }}^{2}\left(E_{k_{\wp}}, \mathbb{Q}_{\ell}(2)\right) \longrightarrow H^{1}\left(k_{\wp}, H_{\text {êt }}^{1}\left(E_{\bar{k}_{\wp}}, \mathbb{Q}_{\ell}(2)\right)\right) .
\end{gathered}
$$

Here we denote by $H^{*}\left(k_{\wp},-\right)$ the continuous Galois cohomology, and the horizontal arrows are the maps which appear in the Leray spectral sequence, taking into account that $H^{0}\left(k_{\wp}, H_{\text {ét }}^{2}\left(\bar{M}_{I / \bar{k}_{\wp}}^{2}, \mathbb{Q}_{\ell}(2)\right)\right)=H^{0}\left(k_{\wp}, H_{\text {ét }}^{2}\left(E_{\bar{k}_{\wp}}, \mathbb{Q}_{\ell}(2)\right)\right)=$ $H^{0}\left(k_{\wp}, \mathbb{Q}_{\ell}(1)\right)=0$; the right vertical arrow is the map induced by $\varphi_{E, I *}$. Since $H^{2}\left(k_{\wp}, H_{\text {ét }}^{0}\left(\bar{M}_{I / \bar{k}_{\wp}}^{2}, \mathbb{Q}_{\ell}(2)\right)\right)=H^{2}\left(k_{\wp}, H_{\text {ét }}^{0}\left(E_{\bar{k}_{\wp}}, \mathbb{Q}_{\ell}(2)\right)\right)=0$ by the local Tate duality, the horizontal arrows are isomorphisms. Since $\varphi_{E, I}$ is non-constant, the homomorphism

$$
H_{\text {ét }}^{1}\left(\bar{M}_{I / \bar{k}_{\wp}}^{2}, \mathbb{Q}_{\ell}(2)\right) \rightarrow H_{\text {ét }}^{1}\left(E_{\bar{k}_{\wp}}, \mathbb{Q}_{\ell}(2)\right)
$$

of $\operatorname{Gal}\left(\bar{k}_{\wp} / k_{\wp}\right)$-modules is split surjective. Hence $\varphi_{E, I, k_{\wp *}}$ is surjective.

\section{Proof of Theorem 1.1}

Let $C, \mathcal{E}, E, S$ be as in the statement of Theorem 1.1. We give a proof of Theorem 1.1 and Corollaries 1.2 and 1.3 in this section. In Section 11.1, we use results from previous sections (Proposition 9.1, and Lemma 10.1) to construct an element in $K_{2}(E)_{\mathbb{Q}}$ for each place of split multiplicative reduction. We keep track of the properties (i.e., under the boundary maps) of the elements using Proposition 3.1 among other things. Note that this construction does not have an analogue in the number field analogue, since there is a clear distinction between the infinity prime and the finite prime in the number field case. Section 11.3 is devoted to the proofs of the statements in the introduction.

11.1. Proof of Theorem 1.1 when there is a section. In this section, we prove Theorem 1.1 assuming that $\mathcal{E} \rightarrow C$ has a section.

When $S=\emptyset$, the target space is zero and there is nothing to prove. Assume $S \neq \emptyset$ and take $s \in S$. We use the notations in Section 9 for $\infty=s$.

For a $k$-algebra $L$, we let $E_{k}=E \times_{\text {Spec } k} \operatorname{Spec} L$ Using the computation done in the proof of Lemma 10.1, we know that $H_{\text {ét }}^{2}\left(E_{k_{\infty}}, \mathbb{Q}_{\ell}(2)\right)=H^{1}\left(k_{\infty}, H_{\text {ét }}^{1}\left(E_{\bar{k}_{\infty}}, \mathbb{Q}_{\ell}(2)\right)\right)$. Since $E$ has split multiplicative reduction at $\infty$, we have $H_{\text {ét }}^{1}\left(E_{\bar{k}_{\infty}}, \mathbb{Q}_{\ell}(2)\right)=V_{\ell}$ where $V_{\ell}$ is as in Section 9.4, and hence $H_{\text {ét }}^{2}\left(E_{k_{\infty}}, \mathbb{Q}_{\ell}(2)\right)$ is one dimensional.

Using Proposition 3.1 and that the dimension of $G_{1}\left(\mathcal{E}_{\wp}\right)$ is less than or equal to 1 by Lemma 2.2, we see that the statement of Theorem 1.1 is equivalent to the surjectivity of

$$
K_{2}(E)_{\mathbb{Q}_{\ell}} \stackrel{\oplus_{\wp} c_{2,2, \wp}}{\longrightarrow} \bigoplus_{\wp} H_{\text {ét }}^{2}\left(E_{k_{\wp}}, \mathbb{Q}_{\ell}(2)\right)
$$

where $\wp$ runs over all primes of $k$.

Combining Proposition 9.1 with Lemma 10.1, we see that the composite

$$
\begin{aligned}
& W_{0} \otimes_{\mathbb{Z}} \mathbb{Q}_{\ell} \rightarrow K_{2}\left(\bar{M}_{\mathrm{lim}, k}^{2}\right) \mathbb{Q}_{\ell} \stackrel{c_{2,2, \infty}}{\longrightarrow} H_{\text {êt }}^{2}\left(\bar{M}_{\mathrm{lim}, k_{\infty}}^{2}, \mathbb{Q}_{\ell}(2)\right) \\
& \stackrel{\varphi_{*, \infty, \mathrm{et}}}{\longrightarrow} H_{\text {ét }}^{2}\left(E_{k_{\infty}}, \mathbb{Q}_{\ell}(2)\right)
\end{aligned}
$$


is surjective. We let $\kappa \in W_{0}$ denote a preimage of a nonzero element in $H_{\text {èt }}^{2}\left(E_{k_{\infty}}, \mathbb{Q}_{\ell}(2)\right)$.

Let us consider the morphism $\varphi_{E, I}$ for a sufficiently small $I$. The homomorphism

$$
\frac{1}{\operatorname{deg}\left(\varphi_{E, I}\right)} \cdot \varphi_{E, I_{*}}: K_{2}\left(\bar{M}_{I / k}^{2}\right) \rightarrow K_{2}(E)_{\mathbb{Q}}
$$

gives rise to the homomorphism $\varphi_{*, K}: K_{2}\left(\bar{M}_{\lim , k}^{2}\right) \rightarrow K_{2}(E)_{\mathbb{Q}}$. We put $\kappa_{s}=$ $\varphi_{*, K}(\kappa) \in K_{2}(E)_{\mathbb{Q}}$.

Lemma 11.1. For $s, s^{\prime} \in S$, we have $\partial_{s^{\prime}}\left(\kappa_{s}\right)=0$ if and only if $s \neq s^{\prime}$.

Proof. For each $s^{\prime} \in S$, we have a commutative diagram

$$
\begin{aligned}
& \begin{array}{cc}
K_{2}\left(\bar{M}_{\lim , k}^{2}\right) \stackrel{c_{2,2, s^{\prime}}}{\longrightarrow} & H_{\text {êt }}^{2}\left(\bar{M}_{\text {lim }, k_{s^{\prime}},}^{2}, \mathbb{Q}_{\ell}(2)\right) \\
\downarrow_{*, K} & \downarrow_{*, s^{\prime}, \text { ét }}
\end{array} \\
& K_{2}(E) \quad \stackrel{c_{2,2, s^{\prime}}}{\longrightarrow} H_{\text {ét }}^{2}\left(E \times_{\text {Spec } k} \operatorname{Spec} k_{s^{\prime}}, \mathbb{Q}_{\ell}(2)\right) .
\end{aligned}
$$

If $s \neq s^{\prime}$, then by construction of $\kappa_{s}$, we have $c_{2,2, s^{\prime}}\left(\kappa_{s}\right)=\varphi_{*, s^{\prime} \text {,ét }}\left(c_{2,2, s^{\prime}}(\kappa)\right)=0$ using Proposition 9.1. By Proposition 3.1, the claim holds in this case.

Suppose $s=s^{\prime}$. Then $c_{2,2, s}\left(\kappa_{s}\right)=\varphi_{*, s^{\prime}, \text { ét }}\left(c_{2,2, s}(\kappa)\right) \neq 0$ by construction. Using Proposition 3.1, we obtain the claim.

Consider the subspace of $K_{2}(E)_{\mathbb{Q}}$ generated by $\left\{\kappa_{s} \mid s \in S\right\}$. Using Lemma 11.1 one sees that it maps surjectively onto the right hand side. This completes the proof of Theorem 1.1 when there is a section.

11.2. Proof of Theorem 1.1. Let $\mathcal{E} \rightarrow C$ be as in the setup of Theorem 1.1. We do not assume the existence of a section to $\mathcal{E} \rightarrow C$. Let $D$ be a projective smooth curve over a finite field with a finite flat morphism $\varphi: D \rightarrow C$ such that the base change $\mathcal{E}_{D}=\mathcal{E} \times_{C} D \rightarrow D$ has a section. Let $\mathcal{E}^{\prime} \rightarrow \mathcal{E}_{D}$ denote a desingularization. The argument above (the proof of Theorem 1.1) applies to $\mathcal{E}^{\prime} \rightarrow D$.

Let $s \in C$ and $t \in \varphi^{-1}(s)$.

Lemma 11.2. We have

$$
\operatorname{dim}_{\mathbb{Q}} G_{1}\left(\mathcal{E}_{s}\right)_{\mathbb{Q}} \leq \operatorname{dim}_{\mathbb{Q}} G_{1}\left(\mathcal{E}_{t}^{\prime}\right)_{\mathbb{Q}} \leq 1 .
$$

Proof. From Proposition 2.1, we have $\operatorname{dim}_{\mathbb{Q}} G_{1}\left(\mathcal{E}_{k_{s}}\right)=\operatorname{dim}_{\mathbb{Q}_{\ell}} H^{2}\left(E_{k_{s}}, \mathbb{Q}_{\ell}(2)\right)$ and $\operatorname{dim}_{\mathbb{Q}} G_{1}\left(\mathcal{E}_{k_{t}^{\prime}}^{\prime}\right)=\operatorname{dim}_{\mathbb{Q}_{\ell}} H^{2}\left(E_{k_{t}^{\prime}}^{\prime}, \mathbb{Q}_{\ell}(2)\right)$ where we write $k^{\prime}$ for the function field of $D$ and $E^{\prime}=E \times_{\operatorname{Spec} k} \operatorname{Spec} k^{\prime}$. We also write $\mathcal{E}_{t}^{\prime}=\mathcal{E}^{\prime} \times_{D} t$ and $E_{k_{t}^{\prime}}^{\prime}=E^{\prime} \times_{\operatorname{Spec} k^{\prime}} \operatorname{Spec} k_{t}^{\prime}$ where $k_{t}^{\prime}$ is the local field at $t$.

The canonical morphism $\varphi_{t s}: E_{k_{t}^{\prime}}^{\prime} \rightarrow E_{s}$ induced from $\varphi$ is finite flat. Since $\varphi_{t s *} \varphi_{t s}^{*}=\operatorname{deg} \varphi_{t s}$ on $H^{2}\left(E_{k_{s}}, \mathbb{Q}_{\ell}(2)\right)$, we obtain the first inequality. The second inequality is the content of Lemma 2.2 .

Let $s \in C$ be a closed point where $\operatorname{dim}_{\mathbb{Q}} G_{1}\left(\mathcal{E}_{s}\right)_{\mathbb{Q}}=1$. Choose an element $t \in$ $\varphi^{-1}(s)$. From the argument in Section 11.1, it follows that there exists an element $\kappa_{t} \in K_{2}\left(E^{\prime}\right)_{\mathbb{Q}}$ such that $\partial_{t^{\prime}} \kappa_{t}=0$ for $t^{\prime} \neq t$ and $\partial_{t} \kappa_{t} \neq 0$. Using Proposition 3.1, we see that $c_{2,2, t^{\prime}} \kappa_{t}=0$ for $t^{\prime} \neq t$ and $c_{2,2, t} \kappa_{t} \neq 0$. 
Let $v \in C$ and consider the following commutative diagram:

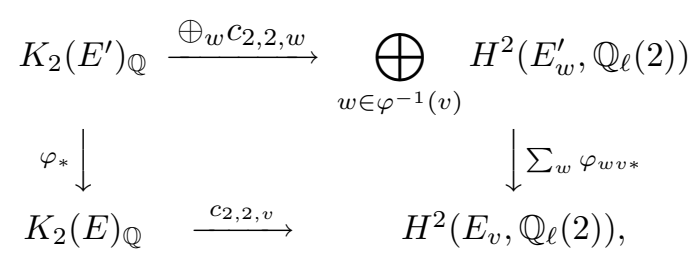

where $\varphi_{w v}$ is the restriction of the morphism $\varphi$.

We then see that $c_{2,2, s^{\prime}} \kappa_{s}=0$ for $s^{\prime} \neq s$ and $c_{2,2, s} \kappa_{s}=0$, since $\varphi_{t s *}$ is an isomporphism if $\operatorname{dim}_{\mathbb{Q}} G_{1}\left(\mathcal{E}_{s}\right)_{\mathbb{Q}}=1$. Using Proposition 3.1, we have the desired properties of the element $\kappa_{s}$ as in the proof of Theorem 1.1 above. The rest of the proof is the same as in Section 11.1. This completes the proof.

\subsection{Proofs.}

Proof of Corollary 1.2. Let $C$ be the projective smooth curve over a finite field whose function field is $k$, and let $\mathcal{E}$ be the flat proper regular minimal model of $E$ over $C$. The claim follows from Theorem 1.1 for $\mathcal{E} \rightarrow C$, after we take (2.1) into account.

Proof of Corollary 1.3. Let $C$ be the projective smooth curve whose function field is $k$. We choose a closed point $c$ and let $A=H^{0}\left(C \backslash\{c\}, \mathcal{O}_{C}\right)$ denote the coordinate ring of the affine smooth curve $C \backslash\{c\}$. It is a Dedekind domain.

Let $\left\{\wp_{i}\right\}_{1 \leq i \leq n}$ be a set of nonzero prime ideals of $A$. Take a nonzero element $a \in \cap_{i} \wp_{i}$. Consider the Weierstrass equation $y^{2}+x y=x^{3}+a$. Note that modulo $\wp_{i}$, it becomes the singular curve defined by $y^{2}+x y=x^{3}$. It has a node at $(0,0)$ with tangent lines $y=0$ and $y+x=0$ (see [Si, p.44, Definition]). Hence the elliptic curve over the function field of $C$ defined by the equation above has split multiplicative reduction at $\wp_{i}$ for each $i$. Now apply Corollary 1.2 to deduce the claim.

\section{REFERENCES}

[Ba-Mi-Se] Bass, H., Milnor, J. W., Serre J.-P.: Solution of the congruence subgroup problem for $\mathrm{SL}_{n}(n \geq 3)$ and $\mathrm{Sp}_{2 n}(n \geq 2)$. Publ. Math., Inst. Hautes Étud. Sci. 33, 59-137 (1967)

[Be] Beilinson, A. A.: Higher regulators and values of $L$-functions. J. Sov. Math. 30, 2036-2071 (1985); translation from Itogi Nauki Tekh., Ser. Sovrem. Probl. Mat. 24, 181-238 (1984)

[B11] Bloch, S.: Algebraic cycles and higher K-theory. Adv. Math. 61, no. 3, 267-304 (1986)

[Bl-Gr] Bloch, S., Grayson, D.: $K_{2}$ and $L$-functions of elliptic curves: computer calculations. In: Bloch, S. J., Dennnis, R. K., Friedlander, E. M., Stein, M. R. (ed.) Applications of algebraic $K$-theory to algebraic geometry and number theory, Boulder/Colo. 1983, Contemp. Math. 55, Part I, 79-88. American Mathematical Society, Providence, RI (1986)

[Bl-St] Blum, A., Stuhler, U.: Drinfeld modules and elliptic sheaves. In: Narasimhan, M. S. (ed.) Vector bundles on curves - new directions, Cetraro (Cosenza), Italy, June 19-27, 1995, Lect. Notes Math. 1649, 110-193. Springer, Berlin (1997)

[Bo] Boyer, P.: Mauvaise réduction des variétés de Drinfeld et correspondance de Langlands local. Invent. Math. 138, no. 3 573-629 (1999)

[Co] Cogdell, J. W.; Kim, H. H.;Murty, M. R., Lectures on automorphic L-functions. Fields Institute Monographs 20. (2004) 
[De-Wi] Deninger, C., Wingberg, K.: On the Beulinson conjectures for elliptic curves with complex multiplication. In: Rapoport, M., Schappacher, N., Schneider, P. (ed.) Bellinson's conjectures on special values of $L$-functions, Oberwolfach, FRG in April 1986, Perspect. Math. 4, 249-272. Academic Press, Boston, MA (1988)

[DeS] De Shalit, E.: Residues on buildings and de Rham cohomology of $p$-adic symmetric domains. Duke Math. J. 106, no. 1, 123-191 (2001)

[Dr1] Drinfeld, V. G.: Elliptic modules. Math. USSR, Sb. 23, 561-592 (1974); translation from Mat. Sb., n. Ser. 94(136), 594-627 (1974)

[Dr2] Drinfeld, V. G.: Elliptic modules. II. Mat. USSR Sb. 31, 159-170 (1977); translation from Mat. Sb., n. Ser. 102(144), no. 2, 182-194, 325 (1977)

[Dr3] Drinfeld, V. G.: Commutative subrings of some noncommutative rings. Funct. Anal. Appl. 11, 9-12 (1977)

[Fu] Fujiwara, K.: A proof of Absolute Purity Conjecture (after Gabber). In Usui, S. (ed.) et al. Algebraic geometry 2000, Azumino. Proceedings of the symposium, Nagano, Japan, July 20-30, 2000, Adv. Stud. Pure Math. 36, 153-183. Mathematical Society of Japan, Tokyo (2002)

[Gek1] Gekeler, E.-U.: A note on the finiteness of certain cuspidal divisor class groups. Isr. J. Math. 118, 357-368 (2000)

[Gek2] Gekeler, E.-U.: On the cuspidal divisor class group of a Drinfeld modular curve. Doc. Math., J. DMV 2, 351-374 (electronic) (1997)

[Gek3] Gekeler, E.-U.: On finite Drinfeld modules. J. Algebra 141, 187-203 (1991)

[Gek4] Gekeler, E.-U.: Über Drinfeldsche Modulkurven vom Hecke-Typ. Compos. Math. 57, no. 2, 219-236 (1986)

[Gek5] Gekeler, E.-U.: Zur Arithmetik von Drinfeld-Moduln. Math. Ann. 262, no. 2, 167-182 (1983)

[Ge-Re] Gekeler, E.-U., Reversat, M.: Jacobians of Drinfeld modular curves. J. Reine Angew. Math. 476, 27-93 (1996)

[Ge-Ja] Gelbart, S., Jacquet, H.: Forms of GL(2) from the analytic point of view. Automorphic forms, representations and L-functions, Proc. Symp. Pure Math. Am. Math. Soc., Corvallis/Oregon 1977, Proc. Symp. Pure Math. 33, 1, 213-251 (1979)

[Gen] Genestier, A.: Espaces symétriques de Drinfeld. Astérisque 234, 124 p. (1996)

[Gi] Gillet, H.: Riemann-Roch theorems for higher algebraic K-theory. Adv. Math. 40, 203-289 (1981)

[Ha] Hamahata, Y.: Tensor products of Drinfeld modules and $v$-adic representations. Manscr. Math. 79, No. 3-4, 307-327 (1993)

[vdHe] van der Heiden, G.-J.: Weil pairing for Drinfeld modules. Monatsh. Math. 143 , no. 2, 115-143 (2004)

[Ja-La] Jacquet, H., Langlands, R. P.: Automorphic forms on GL(2). Lect. Notes Math. 114, Springer-Verlag, Berlin-Heidelberg-New York (1970)

[Ja-Sa] Jannsen, U.; Saito, S., Kato homology of arithmetic schemes and higher class field theory over local fields. Doc. Math., J. DMV Extra Vol., 479-538 (2003)

[Ka-Ma] Katz, N. M., Mazur, B.: Arithmetic moduli of elliptic curves. Ann. Math. Stud. 108, Princeton University Press, Princeton, NJ (1985)

[Ka-Sa] Kato, K., Saito, S.: Unramified class field theory of arithmetic surfaces. Ann. Math. (2) 118, 241-275 (1983)

[Ko-Ya] Kondo, S., Yasuda, S.: Zeta elements in the K-theory of Drinfeld modular varieties, submitted

[La-Mo] Laumon, G., Moret-Bailly, L.: Champs algébriques. Ergeb. Math. Grenzgeb., 3. Folge 39, Springer, Berlin (2000)

[Le] Lehmkuhl. T.: Compactification of the Drinfeld modular surfaces. Mem. Am. Math. Soc. 921,94 p. (2009)

[Liu] Liu, Q.: Algebraic geometry and arithmetic curves. Oxf. Grad. Texts Math. 6, Oxford University Press, Oxford (2002)

[Me-Su] Merkurjev, A. S., Suslin, A. A.: K-cohomology of Severi-Brauer varieties and the norm residue homomorphism. Math. USSR, Izv. 21, 307-340 (1983); translation from Izv. Akad. Nauk SSSR, Ser. Mat. 46, no. 5, 1011-1046 (1982) 
[Mi] Milnor, J. W., Introduction to algebraic K-theory. Annals of Mathematics Studies. No.72. Princeton, N. J.: Princeton University Press and University of Tokyo Press. (1971)

[Qu] Quillen, D.: Higher algebraic $K$-theory I. In: H. Bass (ed.) Algebraic $K$-theory I, Battelle Institute Conference 1972, Lect. Notes Math. 341, 85-147. Springer-Verlag, Berlin-Heidelberg-New York (1973)

[RSS] Rapoport, M. (ed.); Schappacher, N.(ed.); Schneider, P.(ed.) Beilinson's conjectures on special values of L-functions (Based on the meeting of the Arbeitsgemeinschaft Geyer-Harder held in Oberwolfach, FRG in April 1986). Perspectives in Mathematics, 4. Boston, MA etc.: Academic Press, Inc. (1988)

[Ro-Sc] Rolshausen, K., Schappacher, N.: On the second $K$-group of an elliptic curve. J. Reine Angew. Math. 495, 61-77 (1998)

[Sc-Sc] Schappacher, N., Scholl, A. J.: Beilinson's theorem on modular curves. In: Rapoport, M., Schappacher, N., Schneider, P. (ed.) Beilinson's conjectures on special values of $L$-functions, Oberwolfach, FRG in April 1986, Perspect. Math. 4, 273-304. Academic Press, Boston, MA (1988)

[Si] Silverman, J. H., The arithmetic of elliptic curves. 2nd ed. Graduate Texts in Mathematics 106. New York, NY: Springer. (2009)

[St] Stuhler, U.: P-adic homogeneous spaces and moduli problems. Math. Z. 192, 491-540 (1986)

[Th] Thomason, R.W., The Lichtenbaum-Quillen conjecture for $K / \ell_{*}\left[\beta^{-1}\right]$. Current trends in algebraic topology, Semin. London/Ont. 1981, CMS Conf. Proc. 2, No.1, 117-139 (1982)

[vdP-Re] van der Put, M.; Reversat, M.: Automorphic forms and Drinfeld's reciprocity law. Gekeler, E.-U. (ed.) et al., Proceedings of the workshop on Drinfeld modules, modular schemes and applications, Alden-Biesen, Belgium, September 9-14, 1996. Singapore: World Scientific. 188-223 (1997)

[Yu] Yu, J.-K.: Isogenies of Drinfeld modules over finite fields. J. Num. Th. 54, 161-171 (1995)

[EGAII] Éléments de géométrie algébrique. II. Étude globale élémentaire de quelques classes de morphisms. Publ. Math., Inst. Hautes Étud. Sci. 8, 1-222 (1961)

[EGAIII1] Éléments de géométrie algébrique. III. Étude cohomologique des faisceaux cohérents. I. Publ. Math., Inst. Hautes Étud. Sci. 11, 1-167 (1961)

[SGA4-2] Seminaire de geometrie algebrique Du Bois-Marie 1963-1964. Theorie des topos et cohomologie etale des schemas (SGA 4). Un seminaire dirige par M. Artin, A. Grothendieck, J. L. Verdier. Avec la collaboration de N. Bourbaki, P. Deligne, B. Saint-Donat. Tome 2. Exposes V a VIII. Lect. Notes in Math. 270 Berlin-HeidelbergNew York: Springer-Verlag (1972)

[SGA4-3] Théorie des topos et cohomologie étale des schémas. Tome 3. Séminaire de géométrie algébrique du Bois-Marie 1963-64 (SGA 4), Lect. Notes Math. 305, Springer-Verlag, Berlin-Heidelberg-New York (1973)

Satoshi Kondo

Institute for the Physics and Mathematics of the Universe

University of Tokyo

e-mail:satoshi.kondo@ipmu.jp

Seidai Yasuda

Research Institute for Mathematical Sciences

Kyoto University

e-mail:yasuda@kurims.kyoto-u.ac.jp 